Lightning under water: Diverse reactive environments and evidence of synergistic effects for material treatment and activation

Igor Levchenko, Kateryna Bazaka, Oleg Baranov, R. Mohan Sankaran, Alexandre Nomine, Thierry Belmonte, and Shuyan Xu

Citation: Applied Physics Reviews 5, 021103 (2018); doi: 10.1063/1.5024865

View online: https://doi.org/10.1063/1.5024865

View Table of Contents: http://aip.scitation.org/toc/are/5/2

Published by the American Institute of Physics 


\title{
Lightning under water: Diverse reactive environments and evidence of synergistic effects for material treatment and activation
}

\author{
Igor Levchenko, ${ }^{1,2, a)}$ Kateryna Bazaka, ${ }^{2, a)}$ Oleg Baranov, ${ }^{3}$ R. Mohan Sankaran, ${ }^{4}$ \\ Alexandre Nomine, ${ }^{5}$ Thierry Belmonte, ${ }^{5}$ and Shuyan $\mathrm{Xu}^{1, \mathrm{a})}$ \\ ${ }^{1}$ Plasma Sources and Applications Centre/Space Propulsion Centre Singapore, NIE, Nanyang Technological \\ University, Singapore 637616 \\ ${ }^{2}$ School of Chemistry, Physics, and Mechanical Engineering, Queensland University of Technology, \\ Brisbane City, Queensland 4000, Australia \\ ${ }^{3}$ National Aerospace University, Kharkov, Ukraine \\ ${ }^{4}$ Department of Chemical and Biomolecular Engineering, Case Western Reserve University, Cleveland, \\ Ohio 44106-7217, USA \\ ${ }^{5}$ Institut Jean Lamour - CNRS - University Lorraine, Department of Chemistry and Physics of Solids \\ and Surfaces, 2 allée André Guinier, Campus Artem, 54000 Nancy, France
}

(Received 5 February 2018; accepted 27 March 2018; published online 16 May 2018)

\begin{abstract}
This focused review aims to reveal and illustrate some unique features of processes triggered by high-density energy applied to liquids and gas-liquid interfaces and to highlight a wide spectrum of their technological applications capable of producing various advantageous effects, ranging from nanosynthesis to biological and medical applications. Plasma, electric discharges, laser, and ultrasound power effects were selected as representative examples of high-density energy and liquid interactions, yet the available possibilities are not limited by these quite different types of power and thus the reader could extrapolate the outlined features and effects to other kinds of powerful impacts. The basic physical mechanisms are briefly reviewed with the aim to familiarize the readers with the potential capabilities of high-density energy processes in liquids. These will be of direct interest to researchers tasked with the development, optimization, and characterization of processes and highly reactive environments for highly controlled transformation of matter in abiotic and biological systems. It could also be highly useful for under- and post-graduate students specializing in the related fields and general physical audience involved in various plasma, materials, energy conversion, and other concurrent research activities. Published by AIP Publishing. https://doi.org/10.1063/1.5024865
\end{abstract}

\section{TABLE OF CONTENTS}

I. INTRODUCTION $\ldots \ldots \ldots \ldots \ldots \ldots \ldots \ldots \ldots, 1$

II. UNIQUE FEATURES OF PHYSICAL

PROCESSES AT LIQUID INTERFACES. ......

A. Basic configurations and key physical processes...................... 5

B. Electrohydraulic shock (blast) in liquids.... 9

1. Electrohydraulic shock by electric discharges in liquids .............. 9

2. Laser-induced shock waves in liquids ... 10

C. Specific processes in liquids under the action of electric current ..............

1. Electrochemical reactions in liquids interfacing the electrodes and glow discharge plasma ..................

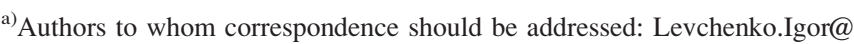
nie.edu.sg; Kateryna.Bazaka@qut.edu.au; and Shuyan.XU@nie.edu.sg
}

2. Solvated electrons at liquids interfacing the electrodes and glow discharge plasma ........................

3. The processes at biological tissues and biofilms interfacing the plasma....... 13

D. Ultrasound effects in liquids ........... 14

E. Self-organization, patterns formation, and adaptive plasma at liquid interfaces ...... 15

F. Synergistic effects in energy-exposed liquids 17

III. APPLICATIONS OF ENERGY-DRIVEN PROCESSES IN LIQUIDS ................

A. Additive processes in liquids: Nucleation and growth ...................... 18

B. Additive processes at plasma-liquid interfaces.......................

C. Subtractive processes at nanoscale: Nanoporous, sharp, and complex structures . 23

D. Bioactivation at plasma-liquid interfaces ... 24

IV. OUTLOOK AND PERSPECTIVES ......... 25

V. CONCLUDING REMARKS .............. 26 


\section{INTRODUCTION}

Novel, sophisticatedly designed and fabricated nanoand metamaterials enable the development of advanced material platforms and metamaterial structures, forming the foundation upon which a large number of innovative technologies, from energy harvesting and storage devices, ${ }^{2}$ miniaturized heat transfer devices, ${ }^{3,4}$ microelectronics, ${ }^{5}$ thin film technology, ${ }^{6}$ metamaterials, ${ }^{7}$ space propulsion technology ${ }^{8,9}$ and many others are built. Impressive results have recently been achieved in these fields, bringing science and technology close to the edge of the next technological revolution, propelled by the advances in nanofabrication technologies and the wide accessibility of information and big data to researchers globally.

However, to realize the most ambitious goals, such as sustainable global food, water, and energy supply; deep space exploration; and extra-terrestrial colonization ${ }^{10,11}$ including Moon and Mars colonization ${ }^{12}$ and global information access via material-enabled technologies, novel material platforms and sustainable, economical, and energy-efficient processes for their fabrication and assembly are required. Their development demands consolidated efforts from distinct fields of science and relies on emerging applications of immense complexity to reach new scientific and technological frontiers. However, mainstream technologies lack energy- and cost-efficiency to sustain the often extreme conditions needed for the synthesis of advanced materials, e.g., pressure of tens of $\mathrm{GPa}$ required for the synthesis of diamonds.

\section{$\checkmark$ Thus, how to efficiently synthesize a unique, meta- stable material?}

Strongly non-equilibrium, high energy processes rapidly deliver precise amounts of power to precursors in complex media to locally create a highly reactive environment under otherwise ambient conditions (e.g., room temperature and atmospheric pressure) and drive otherwise unattainable reactions for self-organized and self-driven material synthesis and activation with high degree of specificity and control. ${ }^{13,14}$ Indeed, when the process is realized within a very short period of time, such as that of a laser pulse or a very short spark-like discharge, strongly intensified nonequilibrium processes could be realized.

Evidently, such localization of extreme pressures, temperatures, and electric fields are particularly well-suited for the large-scale, energy- and cost-efficient, sustainable synthesis, and self-assembly of metastable materials, e.g., nanodiamonds and graphenes, and other unique and/or complex material structures. As an example, Fig. 1 shows a phase diagram of carbon, which depicts very high (as a rule, unattainable under steady-state experimental conditions) pressure and temperature levels required for the synthesis of diamonds. Indeed, the pressure levels of $10 \mathrm{GPa}$ and temperatures of about $5000 \mathrm{~K}$ are required for the efficient synthesis of unique metastable carbonaceous materials. ${ }^{15,16}$

\section{$\checkmark$ Extreme conditions are impractical and often unachievable without non-equilibrium processes}

Successful practical realization of the concept of highly energetic, non-equilibrium synthesis was recently demonstrated by synthesis of various nanoscale materials using nanosecond plasmas. Indeed, it is believed that the unique conditions of such powerful short processes allow for only those building blocks (e.g., atoms) that are necessary for the assembly of the desired material to be produced from the source material, and then rapidly delivered and incorporated into the final structure with high degree of organization and localization to minimize energy consumption and loss. Unlike conventional sequential incorporation of atoms, a process that is both time and energy-consuming, the process described here relies on rapid self-organization. Using an example of synthesis of nanostructured metal oxide materials under ambient air conditions, Fig. 2 illustrates the key requirements and considerations for synthesis driven by nonequilibrium processes. For instance, to attain material efficiency, atoms of oxygen and metal should be generated and incorporated at the exact proportions prescribed by the final material. One way to realize such stoichiometric generation

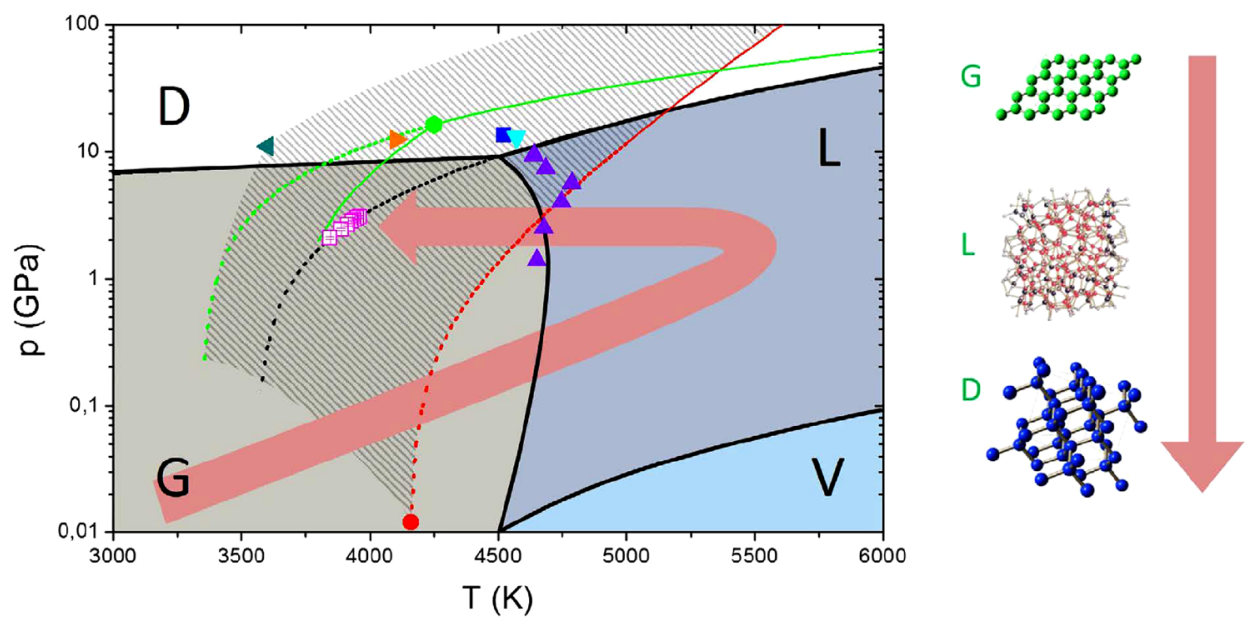

FIG. 1. How to synthesize a unique, metastable material? Extreme conditions are often needed. The phase diagram of carbon features the four regions of diamond D (white), graphite G (light grey), gaseous carbon V (light blue), and liquid carbon L (blue) separated by coexistence lines. The red arrow indicates the evolution of the system: from ambient temperature and pressure to liquid carbon, then to an undercooled liquid and finally to metastable diamond and graphite. See Ref. 1 for more details. Reprinted with permission from F. Gorrini et al., Sci. Rep. 6, 35244 (2016), Copyright 2016 Author(s), licensed under a Creative Commons Attribution 4.0 International License. 

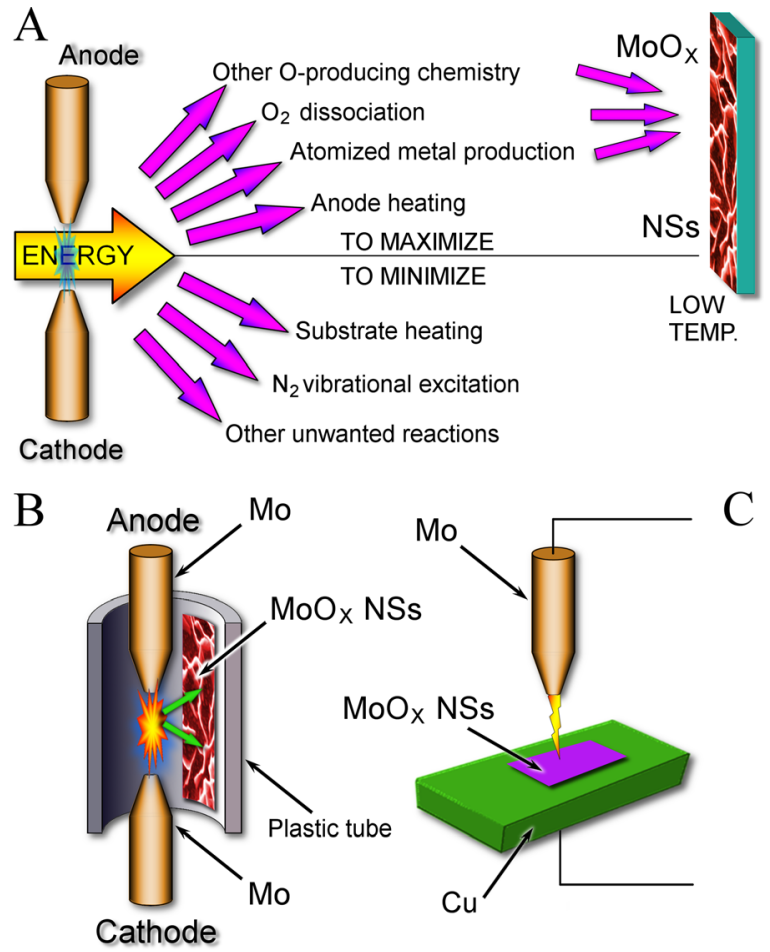

FIG. 2. What are the benefits of power-spike, short-term, strongly nonequilibrium process? Schematics of the experimental setups for the deposition of $\mathrm{MoO}_{\mathrm{x}}$ nanostructures (NS) by nanosecond repetitively pulsed (NRP) spark discharges in open ambient air. (a) The deterministic approach for energy-efficient nanoscale synthesis using atmospheric-pressure plasmas in air. (b) The pin-to-pin electrode configuration for "remote deposition" in which the Mo electrodes act as a source of Mo, and the tube enclosing the electrodes is the substrate for the deposition of $\mathrm{MoO}_{\mathrm{x}}$, and (c) the pin-toplate electrode configuration for "direct deposition" in which the pin electrode is the source of Mo, and the copper plate is the substrate. Reprinted with permission from D. Z. Pai et al., Sci. Rep. 3, 1221 (2013), Copyright 2013 Author(s) licensed under a Creative Commons Attribution 4.0 International License.

of building blocks is by flowing a current between a pair of metallic electrodes in the oxygen atmosphere, where the electrodes and oxygen gas serve as a source of metal and oxygen atoms through evaporation and dissociation, respectively. The relative production of both types of atoms is controlled by fine-tuning the energy of the discharge, where increasing the current of the discharge can lead to highly efficient production of metal vapors with high degree of purity owing to its localization on the surface of the metal electrode in the form of hot spots. ${ }^{17}$

$\checkmark$ Which phase is most suitable for creating extreme conditions using non-equilibrium processes?

The energy applied to solid surfaces result in efficient energy adsorption and material transformation and very dense material fluxes, yet many of the other key processes such as diffusion, heat exchange, and direct delivery of precursors to the growth/synthesis zones are slow. In contrast, in gas phase, many of the said processes, specifically delivery of precursors and diffusion, are quite fast yet relatively low density of precursor materials makes the entire process inefficient.

Complex liquids and carefully designed solutions containing the required precursors along with (potentially) catalysts and other auxiliary admixtures may provide an environment where the advantages of classic liquid-phase organic synthesis, e.g., availability of all reactive groups of the starting materials, can be combined with those of solidphase synthesis, reducing processing time and complexity, and being well-suited for combinatorial chemistry for the discovery of novel materials (Fig. 3).

$\checkmark$ Dense yet fluent, liquids offer a suitable environment for an efficient synthesis of metastable materials.

Indeed, impressive potential of dense power-driven liquid-phase synthesis of nanostructures and metamaterials is reflected by a large number and rich diversity of recent examples in literature, e.g., synthesis of nanodiamonds by pulsed a Nd:YAG laser ablation (with a pulse width of $1.2 \mathrm{~ms}$ and a power density of $10^{6} \mathrm{~W} \times \mathrm{cm}^{-2}$ ) from a suspension of graphite powder in water; ${ }^{18}$ blue luminescent silicon nanocrystals by ns pulsed laser ablation in water; ${ }^{19,20}$ nanodiamonds by femtosecond laser irradiation of ethanol; ${ }^{21}$ $\mathrm{Cu} @ \mathrm{ZnO}$ core-shell nanoparticles by spark discharges in liquid nitrogen; ${ }^{22}$ surfactant-free electrostatically stabilized gold nanoparticles by plasma-induced liquid chemistry; ${ }^{23}$ gold nanoparticle synthesis using an Ar-bubbled plasma setup; ${ }^{24}$ and many others. ${ }^{25,26}$

Various types of energy were successfully applied to liquids to produce useful outcome. Plasma-liquid interactions are among the most promising, and numerous works examine and outline their major types. Saito et al. have examined a wide range of setups that can be used for generating plasmas at liquid interface, which can be classified based on the source used to generate power and the design of the electrodes used to deliver the energy to the discharge region. Using this logic, these setups can be broadly divided into discharges that occur between (i) two electrodes in gas phase; (ii) a metal electrode and the surface of the electrolyte in gas phase; (iii) an electrode and the electrolyte in liquid phase; and (iv) discharges that arise when microwave or

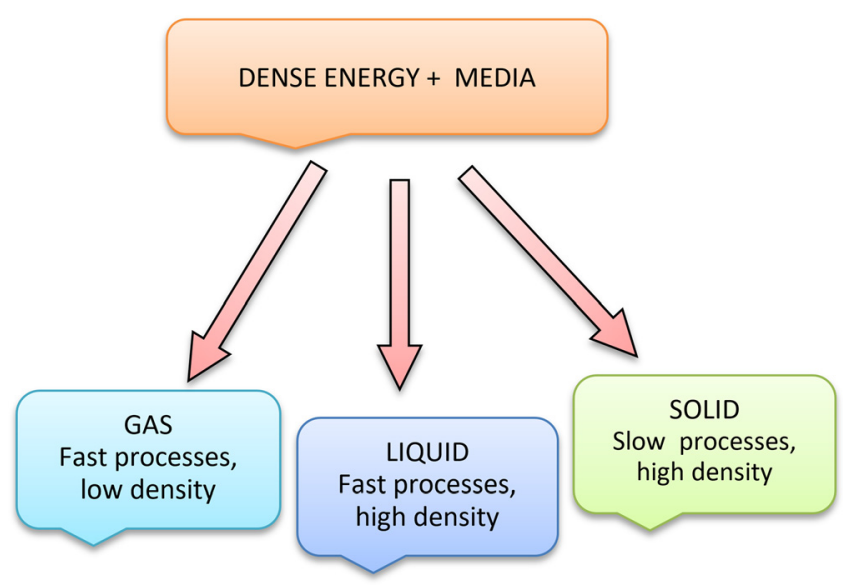

FIG. 3. Why liquid? Benefits and implications of various media. When energy is applied to the surface of a solid, the energy is absorbed efficiently thus enabling the generation of very dense material fluxes, yet other critical processes, e.g., diffusion, heat transfer, and direct delivery of building blocks to the synthesis zone are slower. The gas phase is characterised by a high rate of delivery of precursors and diffusion, yet the lower density of the building blocks renders the process inefficient. Liquids may offer the balanced environment, with pre-set solutions of the precursors, catalysts, and other auxiliary substances. 
(a)

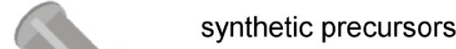

e.g., acetone, acetaldehyde

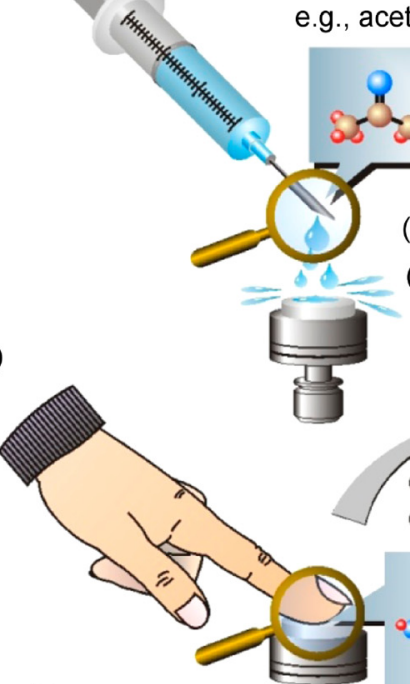

bio precursor

$\left(\mathrm{CH}_{3}\right)_{2} \mathrm{CO} / \mathrm{Ir}(111)$

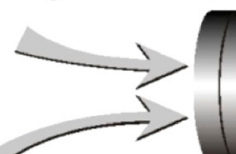

$\mathrm{C}_{x} \mathrm{H}_{y} \mathrm{O}_{z} / \operatorname{Ir}(111)$

$\mathrm{C}_{x} \mathrm{H}_{y} \mathrm{O}_{z} / \mathrm{Rh}(111)$

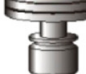

15

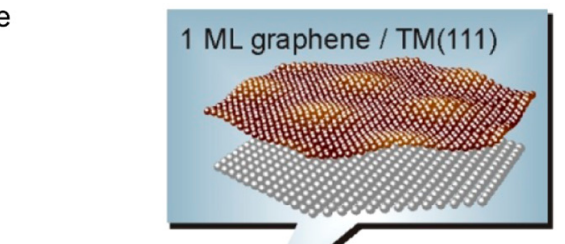
$\left(\mathrm{CH}_{3}\right) \mathrm{COH} / \mathrm{Rh}(111)$
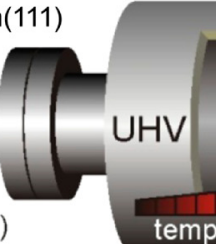

temperature ramping

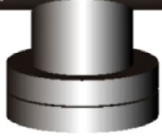

FIG. 4. Liquids as auxiliary media in plasma-based technology: Graphene synthesis via liquid precursor deposition. (a) Ex situ deposition by rinsing a transition metal surface (here, Rh or Ir) with a synthetic precursor (here, acetone or acetaldehyde) is followed by degassing and appropriate temperature ramping up to $\sim 1000 \mathrm{~K}$ in ultrahigh vacuum. (b) Same synthesis route starting with the decoration of the same surfaces with a human fingerprint. Reprinted with permission from F. Müller et al., Langmuir 30, 6114 (2014). Copyright 2008 ACS. radiofrequency energy is delivered into the liquid. These setups offer unique advantages for the synthesis of a variety of inorganic (e.g., Si-based), organic (e.g., C-based), and composite materials in a variety of solutions, including water in the absence of catalyst. ${ }^{27}$

Beyond material synthesis, these processes can benefit biological and medical applications and catalysis, where they are used to potentiate antibacterial activity of small molecules, ${ }^{28}$ introduce biologically and chemically active species into liquids, ${ }^{29}$ and form thinly stratified selforganized patterns at plasma-liquid interface. ${ }^{30,31}$ These can be used to induce specific biological processes in cells (e.g., apoptosis in cancer cells), microorganisms (e.g., enhanced ethanol production by yeast), and plants and animals to increase disease resistance and yield in agriculture and aquaculture.

In addition to direct applications of dense power in the form of laser beam, plasma, or others effects to liquids, indirect involvement of liquid media in the synthesis process (in e.g., thermal furnaces or plasma reactors) also could be very advantageous. Some examples include the production of graphene using liquid precursor deposition technique ${ }^{32}$ (Fig. 4); synthesis of carbon nanostructures for hard tissue engineering involving liquid-enabled stages; ${ }^{33}$ and creation of hybrid carbon nanotube platforms for enzyme immobilization and protection involving liquid precursors. ${ }^{34}$ Another example is shown in Fig. 5, which illustrates the use of pure water as a processing media (which is typically a gas) to generate an arc discharge. In this example, plasma is generated by applying a direct current between a $\mathrm{Cu}$ anode shaped in the form of a nozzle, and an Hf cathode which is housed in a $\mathrm{Cu}$ rod. This system was used to degrade perfluorinated alkenes. One of the key advantages of this setup is that by using pure water in place of more expensive processing gases, it not only lowers the cost of device operation but also obviates the need for the hardware needed for gas supply and
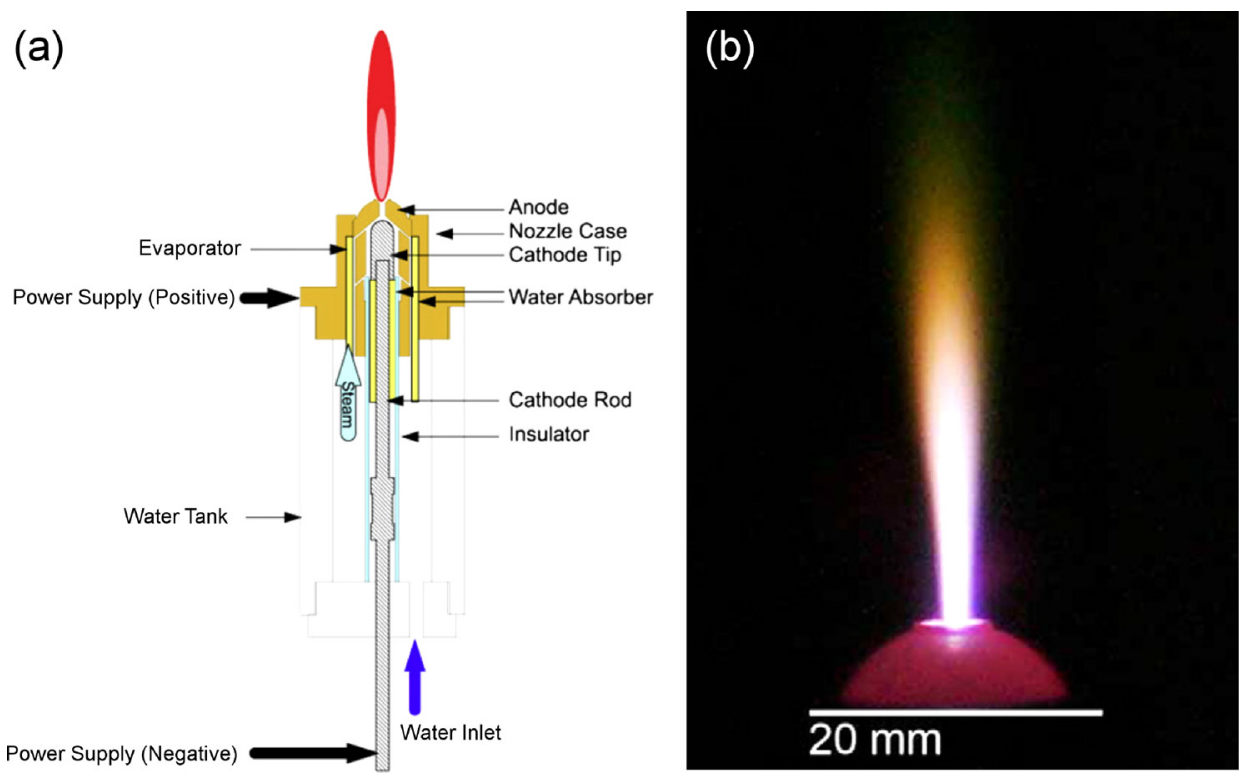

FIG. 5. (a) Schematic diagram of a water plasma torch with DC discharge, used to decompose hydrofluoroethylene by water plasmas. (b) Photograph of a DC water plasma jet. Reprinted with permission from $\mathrm{T}$. Watanabe et al., Thin Solid Films 516, 4391 (2008). Copyright 2008 Elsevier. 
control. Another advantage stems from the fact that auxiliary water cooling becomes unnecessary with this device configuration. ${ }^{35}$

In this focused review, we aim to demonstrate the unusual potential and a wide diversity of highly energetic processes in liquids and at the liquid-gas interfaces for various critically important applications ranging from synthesis of nanoscale and metamaterials, to the biological and medical applications. Unified by the similarity of physical and chemical processes in liquids, these techniques require examination in terms of their outcome, i.e., potential for the diverse applications.

The remainder of this paper is organized as follows. First, we will briefly introduce examples of unique processes in liquids, induced, and being sustained by the application of dense power to liquid-gas interfaces or directly to the liquids. Given the availability of several comprehensive review papers and perspectives that provide an in-depth account of individual physical processes in liquids and liquid-gas interfaces under the action of specific kinds of energy, we will focus on drawing similarities and highlighting key differences in physical processes that arise upon application of different types of power and govern their use in real-life processing of abiotic and living matter. Where necessary referring the reader to seminal works on these topics, we will illustrate specific applications and the key benefits that can be attained from using these processes; specifically focusing on plasma-liquid, laser-liquid, ultrasonic-liquid interactions, as well as electric breakdown in liquid media. Finally, Sec. IV will bring some thoughts on the future trends and current problems facing this rapidly developing technique.

\section{UNIQUE FEATURES OF PHYSICAL PROCESSES AT LIQUID INTERFACES}

\section{A. Basic configurations and key physical processes}

Even when triggered by quite different kinds of power, processes in liquids, or at the gas-liquid interface often demonstrate striking similarity, involving a series of physical and chemical transformations which are virtually independent on the type of energy; these include liquid-gas transformation, ionization, dissociation, and so on. We will examine here several types of the power-liquid interactions, each of the including several sub-types, not too different by the inherent physics. Specifically, the four major setup configurations could be found in various technological applications:

\footnotetext{
- effect of plasma jet on the liquid surface (liquid-gas interface);

- effect of laser beam on the liquid surface;

- effect of ultrasonic energy on liquid; and

- discharges and electric currents in liquids.
}

$\checkmark$ This list does not cover all possible configurations which may use electron beams, ionizing radiation etc.

Importantly, these types of setup configurations and the corresponding types of power exposure are associated with several representative effects, not directly related to the types of power applied, namely (see Fig. 6)

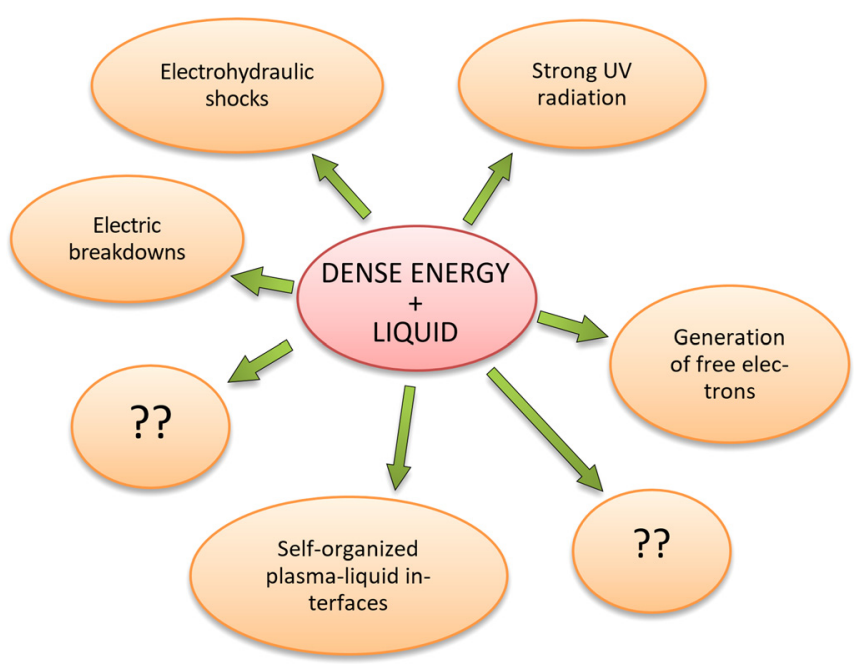

FIG. 6. Examples of strong non-equilibrium processes in liquids under action of high-dense power. The list is open: many processes are still not fully understood and not identified. Further achievements and deeper levels of understanding are anticipated.

1. Electrohydraulic shocks in liquids, caused by, e.g., strong electric currents; propagation of powerful light (laser) radiation; cavitational effects caused by strong ultrasonic power, etc.;

2. Electric breakdown caused by high voltage pulses applied between the two electrodes immersed in liquids;

3. Generation of free electrons and radicals by plasma jet, laser beam, or electric currents interacting with liquid;

4. Exotic excited states of molecules and atoms produced by, e.g., strong UV radiation;

5. And finally, a wide range of other processes and effects that are still not fully explored but apparently plays a significant role in the production of novel materials and treatment and activation of various solutions.

However, this list is essentially incomplete and many other effects and physico-chemical mechanisms have still not been identified and not understood in detail.

It should also be mentioned that the diagnostics of exotic excited states of molecules and atoms produced by, e.g., strong radiation or other powerful, short-pulse effects presents a complex problem and the direct observations, especially at the very beginning of the breakdown, are still a challenge. Theoretical approaches may be a key here to look closer into this processwhich ions are formed, how long can they exist, etc. ${ }^{36}$

Figure 7 shows a graphical representation of the four above-mentioned main configurations, with the characteristic nature of the respective power-liquid interfaces. These are just representative examples, and the reader is encouraged to explore the rich diversity of possible variations in the dedicated high-quality comprehensive reviews; e.g., a review by Saito et al. which provides an excellent outline of liquidplasma configurations. ${ }^{27}$

Hybrid configurations that utilize combinations of several types of energy are also possible. Importantly, synergistic effects could be found in such hybrid setups that are capable of producing unique structures, nano- and metamaterials, or demonstrate extremely high efficiency, productivity, 


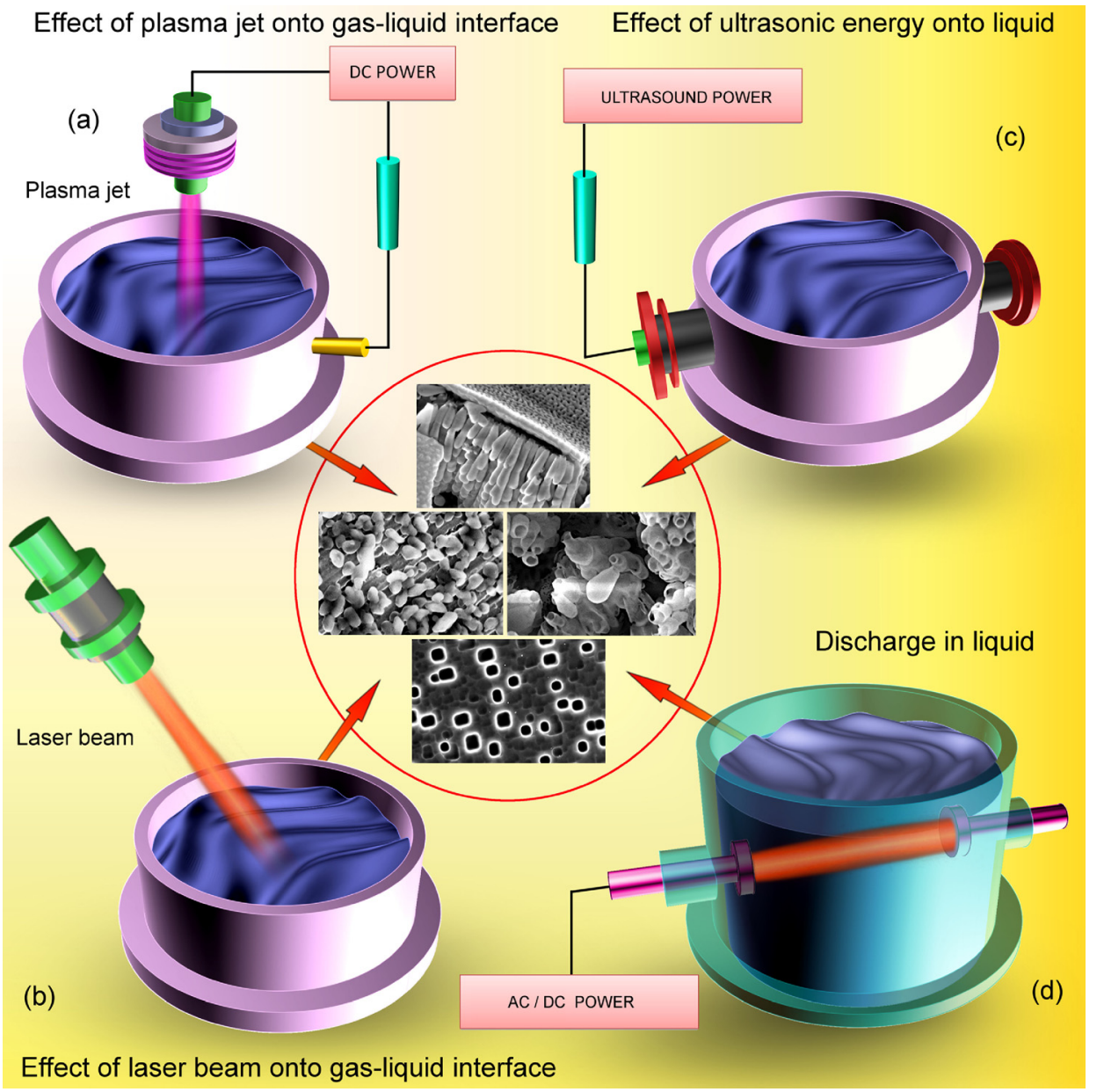

FIG. 7. Four major typical configurations of power-liquid interfaces. (a) Plasma jet directly interferes with the liquid surface at the liquid-gas interface. Electric current passes through the plasma and then liquid, finally collected to the second electrode (usually immersed in liquid); setups with the two plasma jets are also possible but were not tested so far; (b) laser beam directly interferes with the liquid surface; (c) ultrasonic energy is supplied to liquid; and (d) discharge or strong electric current is passed in liquids through the two immersed electrodes. All the above configurations are able to produce unique structures and other useful outcomes. SEM images at the insets are reprinted with permission from J. Fang et al., IEEE Trans. Plasma Sci. 43, 765 (2015). Copyright 2015 IEEE; and from J. Fang et al., Sci. Technol. Adv. Mater. 15, 045004 (2014); Copyright 2014 National Institute for Materials Science, licensed under a CC-BY-3.0 License.

and other remarkable features. As illustrated, combinations of ultrasonication and electrochemical processes, or laser and electrochemistry, or ultrasonication with laser may be implemented. Tables I and II list several elemental processes, the relevant outcomes, and hybrid schemes potentially capable of creating synergistic effects (i.e., effect arising between two or more agents, entities, factors, or substances that produces an effect greater than the sum of their individual effects); some of these schematics will be examined in this review. ${ }^{37}$

Synergistic effects demonstrate experimentally proven capabilities in many fields ranging from medical applications, ${ }^{38}$ activation and enhancing the action of antibiotics, ${ }^{39}$ and targeted therapy, ${ }^{40}$ to various technical and technological applications such as core-shell nanowire arrays for high energy and power density lithium-ion batteries, ${ }^{41}$ fabrication of fatigue resistant graphene-based nanocomposite via synergistic interfacial interactions, ${ }^{42}$ sophisticatedly designed nanoparticles for efficient photocatalytic hydrogen production, ${ }^{37}$ and many others. Not aiming to a comprehensive review of the whole spectrum of synergistic effects that could be implemented in the energy-exposed liquids, we will illustrate the possible combinations and potential possibilities of the synergy-appropriate systems.

\section{$\checkmark$ Synergy may be a key factor for breakthrough in the innovative material synthesis}

It should be noted that numerous recently published original works and reviews discuss in great detail various specific features of physical processes under the action of highly dense power onto liquids. Belmonte et al. gives a comprehensive outline of the interaction of electric discharges with electrode surfaces in dielectric liquids, with the detailed typology of discharges, which are classified based on their electrical characteristics, liquids, and setup configurations. ${ }^{43}$ Cheng et al. described in detail the processes during plasma electrolytic oxidation (PEO), including the mechanisms of internal sparking and the effect of electrolyte composition. ${ }^{44}$ Kareem et al. have examined the application of glow discharge plasma electrolysis for nanoparticles synthesis. ${ }^{45}$ Tochikubo et al. examined the liquid-phase reactions induced by atmospheric pressure glow discharge with a liquid electrode. ${ }^{46}$ Synthesis of magnetic nanoparticles by atmospheric-pressure glow discharge plasma-assisted electrolysis was recently reviewed by Shirai et al. ${ }^{47}$ Giridhar et al. have demonstrated the electrodeposition of nanocrystalline aluminum from ionic liquids. ${ }^{48}$

Plasma-liquid and specifically microplasma-liquid interfaces were also outlined in several comprehensive reviews. Mariotti et al. have presented a detailed discussion on the processes in liquid under the microplasma, yet did not present many examples of applications for such systems for material synthesis. ${ }^{49}$ Bruggeman et al. have also presented a detailed review on the processes at plasma-liquid interactions, with only a limited discussion of the examples of the use of these systems. ${ }^{50}$ In contrast, Lin et al. have presented 
TABLE I. Elemental processes, examples of outcomes, and specific physical processes intrinsic to each configuration.

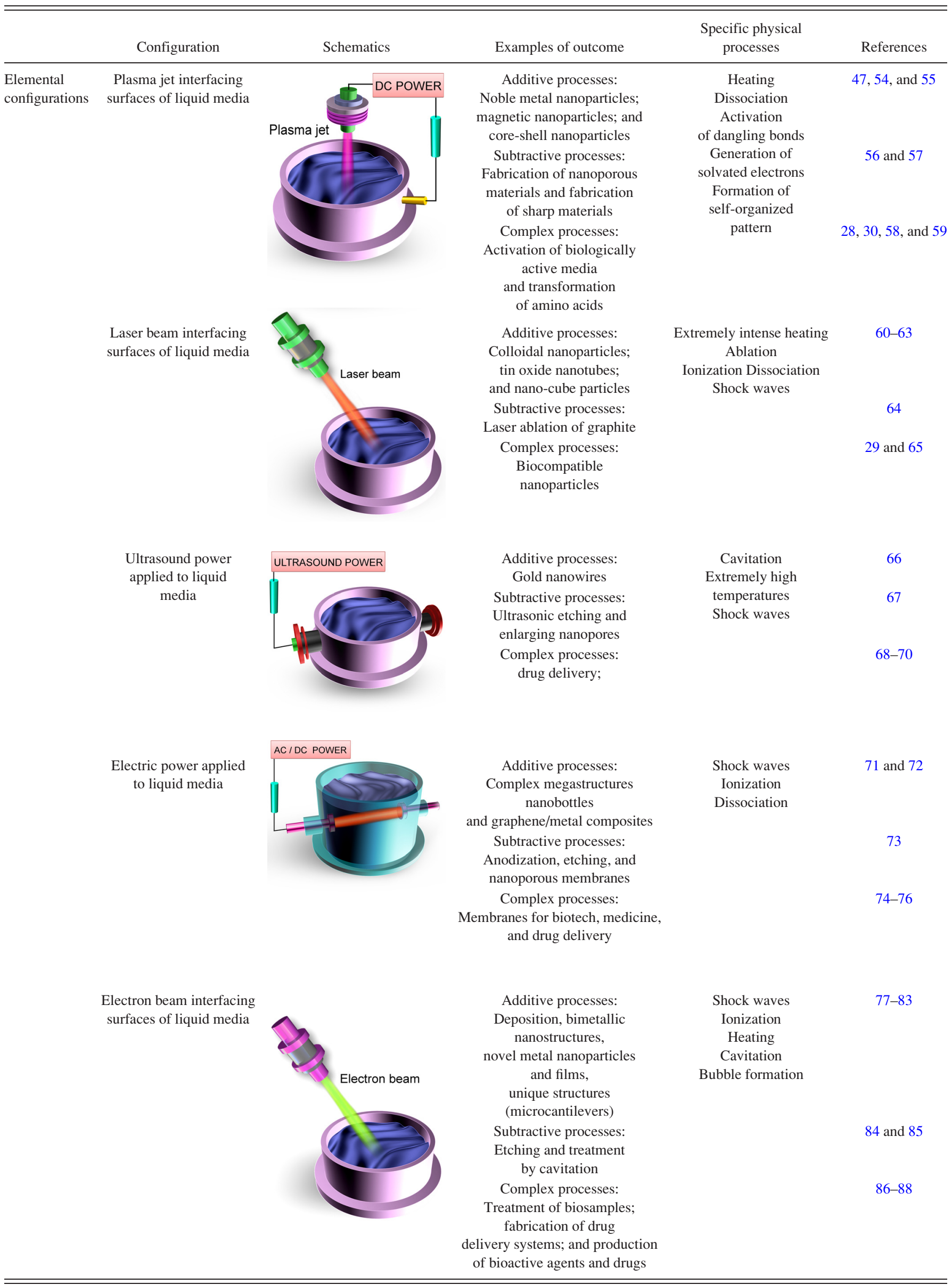


TABLE II. Hybrid schemes potentially capable of creating synergistic effects and examples of outcomes found for each hybrid scheme.

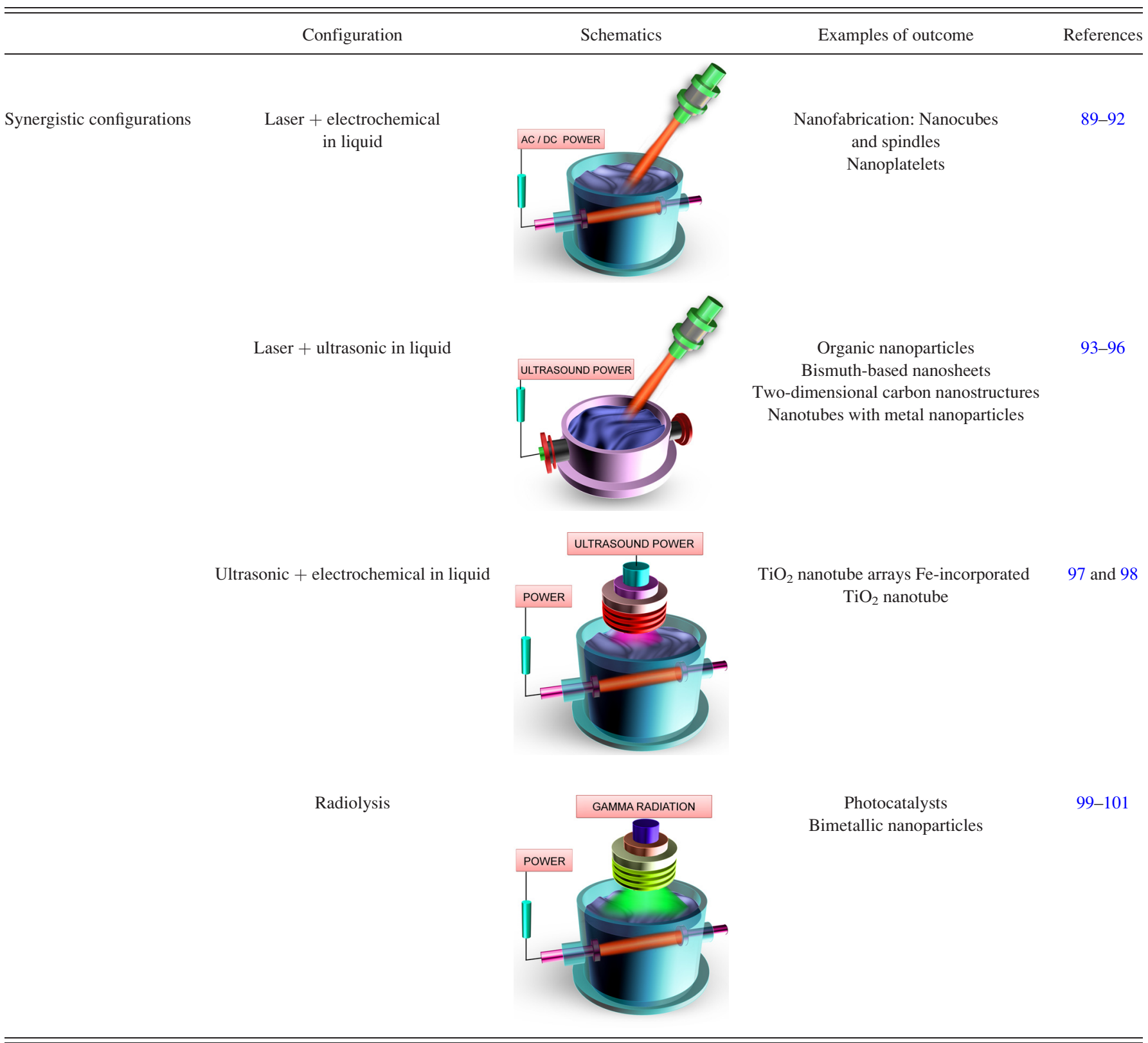

an impressive outline of the spectrum of new generation of technologies for the microplasma-enabled functional nanomaterial synthesis, including those involving the liquid precursors; ${ }^{51}$ Mariotti et al. have also presented a review of technological microplasma-based systems with liquid media, ${ }^{52}$ while Belmonte et al. concentrated on the nonequilibrium processes in plasma-liquid interactions. ${ }^{53}$

Laser-liquid interactions and the associated effects were addressed in reviews by Kruusing et al. where the physics and chemistry of laser-liquid-solid interactions were examined, ${ }^{102}$ along with the laser-induced shock processing. ${ }^{103}$ Radu et al. have analyzed silicon treatment by etching in various liquid precursors by femtosecond laser pulses, thus utilizing very fast, non-equilibrium processes; ${ }^{104}$ other authors have considered the laser-liquid-solid interactions for synthesizing alloys and nanostructures from liquid precursors. ${ }^{05,106}$ Tarasenko et al. have presented the analysis of the laser-enabled synthesis of composite nanoparticles in liquids, ${ }^{107}$ and Švrček et al. have demonstrated the synthesis of blue luminescent nanocrystals by nanosecond-pulsed laser ablation in water. ${ }^{108}$

Specific processes were also addressed in specially devoted research works. For example, charge accumulation at the electrolyte-oxide interface during the plasma electrolytic oxidation was examined by Martin et al. ${ }^{109}$ and Nominé et al., ${ }^{110}$ along with the high-frequency-induced cathodic breakdowns. ${ }^{111}$ Other authors have studied, e.g., the statistics of plasma discharge parameters (Dunleavy et al. ${ }^{12}$ ) and individual discharge cascades (Troughton et $a{ }^{113}$ ). Ghosh et al. have examined the generation of a direct-current, atmospheric-pressure microplasma at the surface of a liquid water microjet. ${ }^{114}$

Another exciting example of the specific plasma-liquid interaction mechanism is the formation of a self-organized anode pattern on the surface of a liquid or metal anode. ${ }^{115}$ Impressive features and promising possibilities of the 
adaptive and self-adaptive approaches based on selforganized mechanisms of plasma-liquids were recently demonstrated. ${ }^{30,116}$ It should be noted that complex physical processes are involved in self-organization and other self-driven effects. $^{3,13,117}$

Thus, the readers could find a wide range of literature on various aspects and specific processes involved and caused by quite different types of energy applied to liquids and gasliquid interfaces. In contrast, in this article, we will focus on the big picture approach to analyze the unifying features of the aforementioned processes and their applications, generalized by the application of highly dense energy exposures to liquid media and precursors.

Below, we will examine some processes and effects that are most attractive for the material treatment and activation, illustrate the rich spectrum of possibilities of these techniques in the effort to entice the reader to seek deeper understanding of the underlying physics.

\section{B. Electrohydraulic shock (blast) in liquids}

Electrohydraulic shocks are among the most interesting and promising processes that could be realized by exposing liquids to quite different kinds of energy, from electric discharges and laser pulses to even mechanical impacts; however, the physical pictures of the shock developing in the liquid feature quite similar behavior and hence demonstrate quite comparable potential for the material treatment, synthesis, and media activation.

\section{Electrohydraulic shock by electric discharges in liquids}

Figure 8 illustrates the development of the pulsed-spark electrohydraulic-discharge plasma in aqueous solutions containing sodium sulfate or potassium chloride. The energy is delivered in the form of a bipolar pulsed voltage of high magnitude and frequency by means of two rod-shaped electrodes separated by a several mm-wide gap. The application

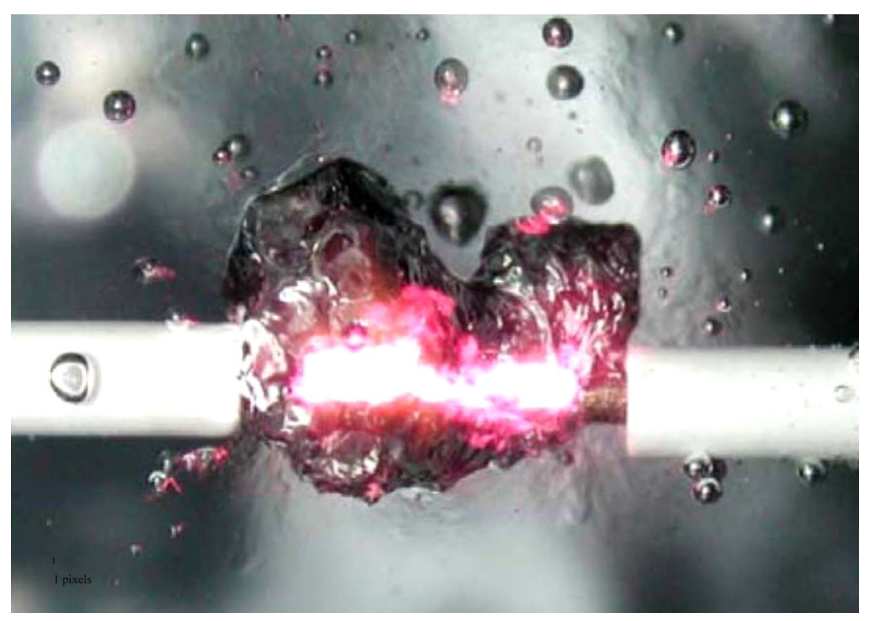

FIG. 8. Morphology of high-frequency pulsed-spark electrohydraulicdischarge liquid-solution plasma for a discharge gap distance of $3 \mathrm{~mm}$ and a maximum pulse voltage of $1.5 \mathrm{kV}$ at a frequency of $15 \mathrm{kHz}$. Steady-state bridged discharge $\mathrm{t}=6 \mathrm{~s}$. Reprinted with permission from T. Takeda et al., IEEE Trans. Plasma Sci. 36, 1158 (2008). ${ }^{118}$ Copyright 2008 IEEE. of the voltage triggers the transformation of the joule-heated water into steam, at which point the spark discharge is formed at the edge of the two electrodes. Owing to a very short length of individual pulses $(2 \mu$ s at $10 \mathrm{kHz})$, the establishment of the process proceeds over a number of small spark discharges, as demonstrated by the evolution of the heat profile. Optical spectral measurements show that the process results in the formation of large quantities of reactive species, including $\mathrm{H}, \mathrm{OH}, \mathrm{H}_{2} \mathrm{O}_{2}, \mathrm{O}_{3}$, and $\mathrm{O}$. While species such as $\mathrm{H}_{2} \mathrm{O}_{2}$ will undergo further dissociation to $\mathrm{H}$ and $\mathrm{O}$, the fractions of $\mathrm{OH}$ and $\mathrm{O}_{3}$ do not change significantly over time of the processing. Apparently, an extremely reactive environment is formed under those conditions, satisfying the prerequisites for the synthesis of unique structures and obtaining very active liquid media.

Moreover, the discharge-produced pressure could reach $1 \mathrm{MPa}$ for the pulsed arc types of discharge, along with the strong UV generation. ${ }^{119}$ In the case of high-voltage discharge, even stronger effects are produced. Indeed, a pulse voltage applied to the electrodes in an aqueous solution creates a plasma channel of very high conductivity, in which the pressure can reach several GPa when the resistance of the channel reaches its minimum. As a consequence, the channel expands rapidly, resulting in the formation of a cavity within the solution, and subsequently an ultrasound pulse of a significant magnitude. The extent of the cavity expansion depends on the characteristics of the power source used to ignite the discharge and those of the plasma channel. The ensuing collapse of the cavity results in another acoustic pulse. $^{120}$ The most recent studies have demonstrated the pressure levels reaching $10 \mathrm{MPa}$ in the dielectric liquids for the discharge energy of about $1000 \mathrm{~J} .{ }^{122}$ One more important factor of the shock wave in liquid is the formation of a wide range of high-frequency oscillations at the megahertz levels. ${ }^{121}$ Apparently, these oscillations are capable of effectively exciting and activation molecules and complexes in liquid solutions.

Important relations were obtained experimentally for the shock wave intensity as functions of the plasma channel length and energy deposited into the arc channel ${ }^{122}$

$$
P_{m}=0.58 \times L_{a}^{0.75}, \quad P_{m}=0.26 \times E_{p l}^{0.54},
$$

where $L_{a}$ is the plasma channel length, $\mathrm{mm} ; E_{p l}$ is the energy deposited in the plasma breakdown, and Pm is the peak pressure in the shock wave. Apparently, quite high pressure levels exceeding $10 \mathrm{MPa}$ demonstrate strong potential for various applications. Figure 9 illustrates the relationship between the peak current, plasma channel length, and shock wave.

Wire explosion by the action of very strong electric current pulses in a liquid media is also capable of producing the powerful electrohydraulic shocks. For the experiments in the mixture of water and glycerine and the energy released in the wire of about $1 \mathrm{~kJ}$, the mean shock wave velocity reached $700 \mathrm{~m} / \mathrm{s}$, with the maximum shock wave pressures up to $50 \mathrm{MPa} .{ }^{123}$ Usability of this method was proved by several researchers by demonstrating, e.g., the synthesis of $\mathrm{CuO}$ nanocrystals via pulsed wire explosion in liquid ${ }^{124}$ and production of nanopowders by wire explosion in water. ${ }^{128}$ The 


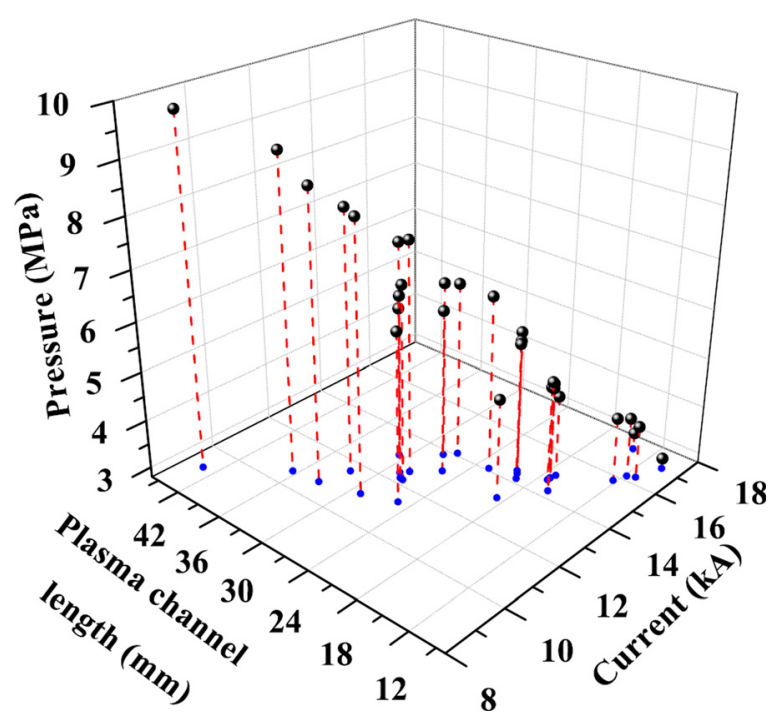

FIG. 9. Relationship between the peak current, plasma channel length, and shock wave during breakdown in dielectric liquid. Strong pressure levels reaching $10 \mathrm{MPa}$, and very high peak current up to $15 \mathrm{kA}$ could be produced in the high voltage breakdowns of the liquids. Reprinted with permission from Phys. Plasmas 24, 043510 (2017). ${ }^{122}$ Copyright 2017 AIP Publishing LLC.

typical picture of the shock wave development after wire explosion in water is illustrated in Fig. 10.

\section{Laser-induced shock waves in liquids}

The laser-induced shock (blast) waves represent the next important example of highly dense energy application that results in the very strong process conditions. Here, plasma is created as a result of direct ionization (as multiple photons are absorbed by the media simultaneously) or avalanche ionization (which relies on the frequent collisions between (quasi-)free electrons and the media). ${ }^{125,126}$ The characteristics of the liquid media, e.g., its ionization energy and chemical composition, and those of the incident laser beam, e.g., beam divergence, pulse, and wavelength will determine the minimum value of the radiant flux per a surface unit area required for the generation of plasma. The value of the threshold of the laser-induced breakdown in solutions can be estimated based on experimental observation of the emitted plasma, generation of bubbles in the liquid, and phenomenological analysis of the released shock waves. It should be noted that physically, the laser breakdown is also a plasma- based phenomenon, thus emphasizing the general physical nature on many processes that occur in liquids under the actions of various highly dense power exposures.

Among other important effects, self-focusing attracts a special attention due to enormous and still unclear potential intrinsic to many self-organized processes. Because of nonlinearity of refractive index in liquid media which acts as a focusing lens, self-focusing in the form of filamentation due to Kerr's effect may arise as radiation propagates in liquid, with the mechanism dependent on both the intensity of the electromagnetic radiation and the characteristics of the media through which it propagates. For small focusing angles and the radiation intensity $I$, the variation in the refractive index can be expressed as

$$
n=n_{0}+n_{2} I,
$$

where $n_{0}$ and $n_{2}$ denote wavelength-dependent linear and nonlinear refractive indices, respectively. The latter value is typically positive, leading to greater $n$ in the regions with larger radiation intensity (e.g., center of the beam), thus producing a focusing density profile along the path of the beam.

The energy of bubble produced by the laser pulse can be calculated using a classical Rayleigh's equation

$$
E_{b}=\frac{4 \pi}{3}\left(p_{0}-p_{v}\right) R_{\max }^{3}
$$

where $R_{\max }$ is the radius of the bubble at the point of maximum expansion, $p_{0}$ is the hydrostatic pressure, and $p_{v}$ is the vapor pressure inside the bubble; note that $p_{v}$ is the descriptive value that is determined by the specific media. ${ }^{129,130}$

Laser-induced breakdown of liquid column represents one more useful and physically interesting example of laserproduced shocks in liquids. In this case (see Fig. 11), the application of focused radiation onto a liquid micro-column produces a shock wave of much greater intensity than that produced in air, ${ }^{130}$ thus revealing strong technological potential and interesting physical background of these effects.

Breakdowns in cryogenic liquids by laser pulses were investigated just recently. Surprisingly, the breakdowns in liquid helium have demonstrated a well-pronounced memory effect, which consists of a relatively long lasting (several seconds) increase in the likelihood that the next pulse will result in a breakdown. For the laser (Nd:Yag, 532- and 355$\mathrm{nm}$ beams) pulse duration from 3 to $5 \mathrm{~ns}$, there was a near
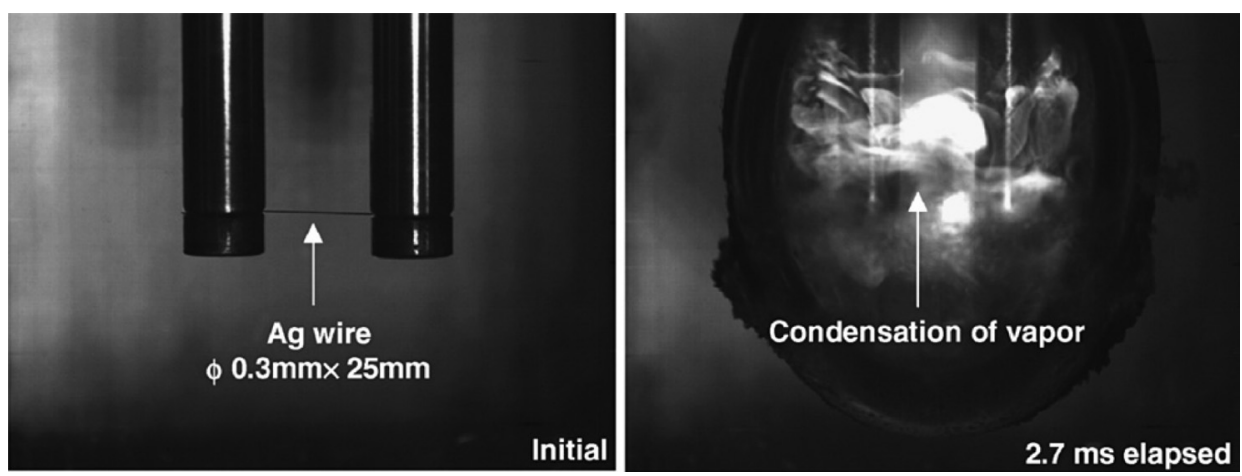

FIG. 10. High-speed camera images of a silver wire explosion in water $(10$ $\mu \mathrm{F}, 12 \mathrm{kV}, 3000 \mathrm{pps}$ of frame speed, and $300 \mu$ s of exposure). Reprinted with permission from C. H. Cho et al., Surf. Coat. Technol. 201, 4847 (2007). ${ }^{128}$ Copyright 2007 Elsevier. 


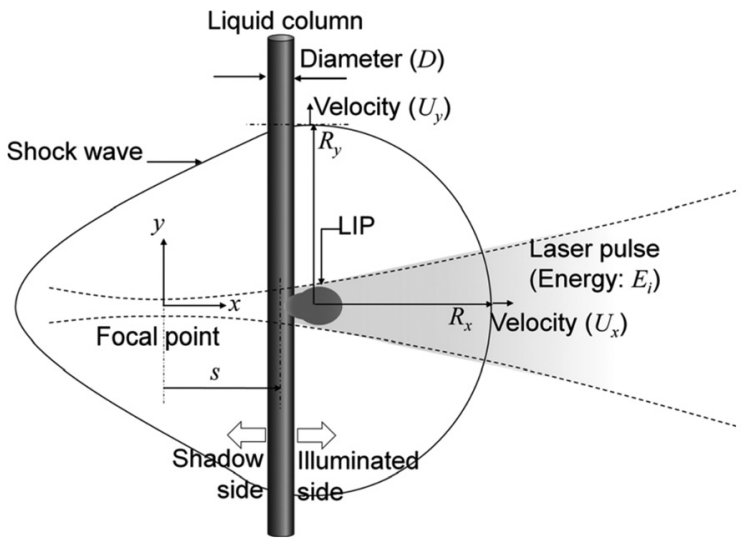

FIG. 11. Schematic of laser-induced breakdown of liquid column. Impressive values of the shock wave velocities reaching $3500 \mathrm{~m} / \mathrm{s}$ were obtained in the experiments with the laser pulse energy of $300 \mathrm{~mJ}$ and time scale of $500 \mathrm{~ns}$. Reprinted with permission from J. Appl. Phys. 109, 073101, 2011. ${ }^{127}$ Copyright 2011 AIP Publishing LLC.

$3 \times$ drop in the minimum power required for the liquid breakdown. ${ }^{131}$ Helium is a neutral, noble substance which cannot form any complexes and chemical compounds; however, the memory effect suggests the formation of long-living structures (presumably, the dimers which feature much lower ionization energy).

The blast waves could be produced by a laser or other energy pulses in the media of liquid particles suspended in a gas (aerosols). While not a true liquid media, such environments are very promising for a wide range of applications, from nanosynthesis ${ }^{132}$ to cancer therapy by the activated liquid suspensions. ${ }^{133,134}$ The presence of liquid particles in the shock-carrying environment essentially changes the entire dynamics of the process.

The shock waves that can be produced in this complex environment of suspended particles can be broadly divided into four categories based on their structure. The first structure is produced as a result of the interactions between the particle and the beam when the incident radiation impacts the particle above the ideal focal spot. In the second type of breakdown, the incident beam impacts on a smaller number of particles at the upper breakdown point; whereas the opposite is the case for the third type of the breakdown, where a greater number of particles is impacted. In all of the aforementioned types, the evolution of the gas breakdown is associated with the occurrence of only two independent waves. In contrast, in the fourth (triple) type of breakdown, the breakdown is associated with one additional blast wave. ${ }^{135}$

\section{Specific processes in liquids under the action of electric current}

While electrohydraulic shocks encompass a spectrum of processes resulting in mechanical (pressure spikes and shock waves), thermodynamic (temperature spikes), and other auxiliary effects like UV radiation, the processes in liquids caused by the electric current below the "blast" threshold (i.e., those that do not cause discontinuity and cavitation in liquid media), but still strong enough to cause significant changes in the media, are of a special importance. Below, we will examine some representative processes and effects that will describe the complexity and usefulness of these processes for practical applications.

\section{Electrochemical reactions in liquids interfacing the electrodes and glow discharge plasma}

Early experimental investigations of the interactions between plasmas in the glow mode and liquids were conducted using an electrode, typically a conductive wire of copper, gold, platinum, or tungsten, suspended above the surface of the liquid. The second electrode of a greater area, e.g., a thin sheet of metal, mounted in the solution would complete the circuit, as depicted in Fig. 12(a). The small diameter of the wire enables the electric field to be higher at that electrode. Figure 12(b) shows typical current-voltage (IV) characteristics of the above described setup.

The discharge is generated in the gas phase when a voltage, typically dc, is applied to the setup, where the plasma electrode can act as an anode or as a cathode in relation to the immersed electrode. The species produced by the plasma then interact with the liquid at the interface, with the extent of interactions defined by the volume of the plasma and the depth to which plasma species can penetrate into the solution. Heating, gas flow, and bombardment by energetic species often lead to evaporation, sputtering and spraying of the molecules from the interface, resulting in some quantity of the liquid media to be suspended in the gas phase, and as such be directly affected by the glow discharge, e.g., through ionization.

In the second typical embodiment of such a system, the plasma-generating electrode is brought into direct contact with the liquid phase, as depicted in Fig. 12(c). Similar to the previous configuration, the plasma electrode can act as an anode or as a cathode. In the case of the former, the properties of the surface of the plasma-generating electrode play a less important role ${ }^{137}$ compared to the properties of the liquid phase, particularly its conductivity. ${ }^{138}$ Anodic discharges have been successfully demonstrated in solutions of different $\mathrm{pH}$, from strongly alkaline (e.g., sodium hypophosphite ${ }^{137}$ ) to more acidic (e.g., sodium chloride acidified with, e.g., $\mathrm{HCl}$ ). To minimize evaporation, the solution is kept at $30-60^{\circ} \mathrm{C}$, although the temperature can rise during plasma treatment owing to resistive heating. A characteristic I-V curve for this type of plasma discharge is presented in Fig. 12(d).

Chemical reactions that take place at the interface of an electrolyte solution and a metal electrode are relatively well understood and can be broadly divided into reduction reactions at the cathode and oxidation reactions at the anode, as shown in Figs. 13(a) and 13(b) for the example of water reduction and oxidation to produce $\mathrm{H}_{2}$ and $\mathrm{O}_{2}$ gas, respectively. In contrast, the interface of an electrolyte solution and a plasma electrode is extremely complex, and despite a long history, remains very poorly understood. Thus, a focus of recent research has been to understand the physicochemical processes in liquid media that are in contact with a plasma that contains electrons, ions, radicals, 

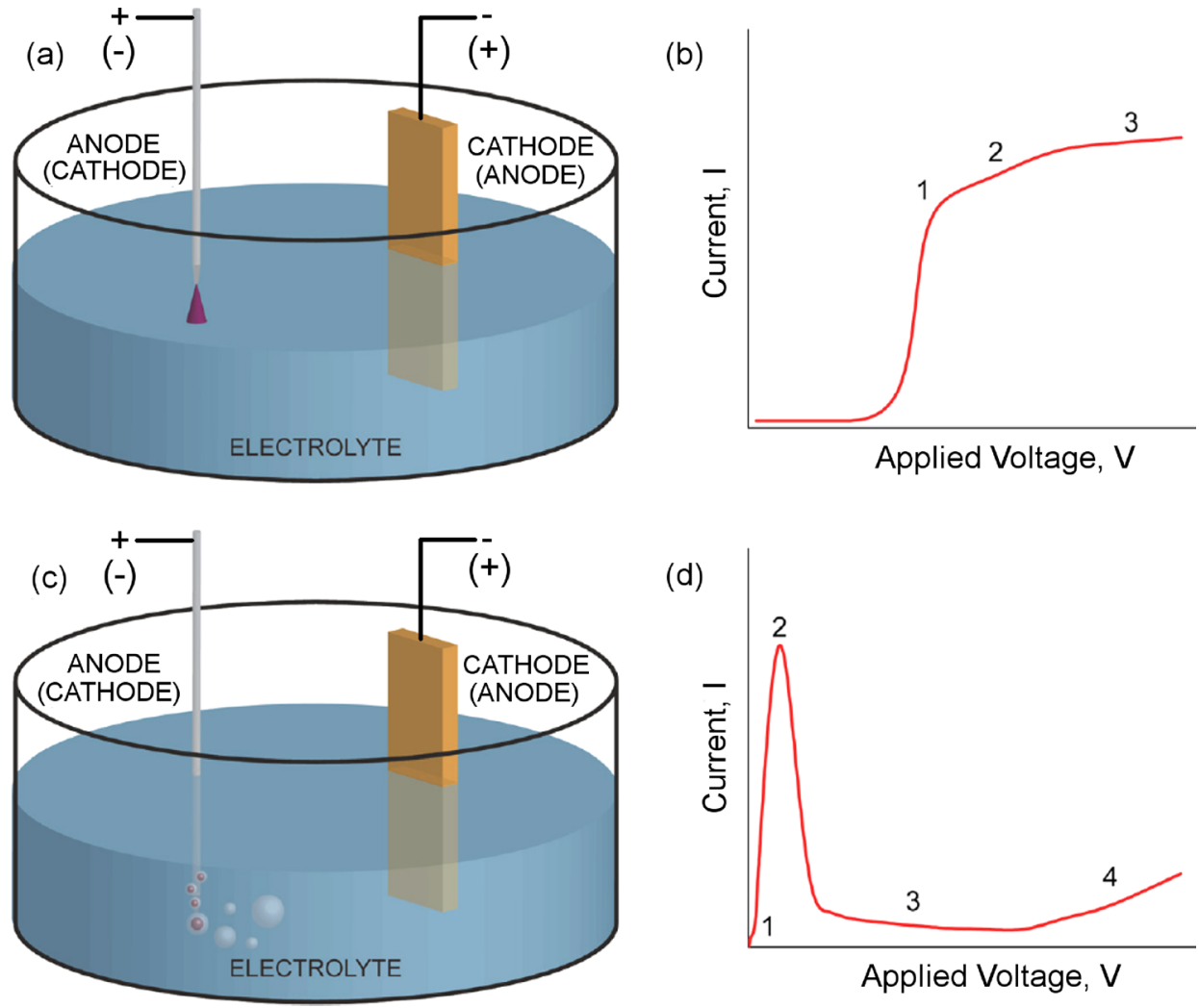

(d)

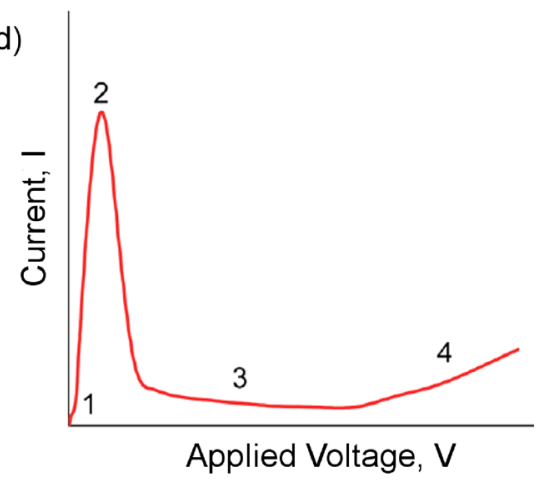

FIG. 12. Typical experimental configurations and corresponding characteristic current-voltage (I-V) curves for [(a) and (b)] GDE and [(c) and (d)] CGDE, respectively. Reprinted with permission from R. Akolkar et al., J. Vac. Sci. Technol. A 31, 050811 (2013). ${ }^{136}$ Copyright 2013 AVS. and photons, and in some cases supports the passage of electric current into the liquid. In particular, one of the unique features of plasma-liquid interactions is the possible generation of solvated electrons by the injection of gasphase electrons from the plasma into the liquid phase. Solvated electrons are extremely active species and (being the smallest possible anions) play a significant and sometimes a key role in the formation of active solutions, for water remediation and medical applications.

\section{Solvated electrons at liquids interfacing the electrodes and glow discharge plasma}

Owing to their unmatched reducing potential and yet-tobe-resolved structure, solvated electrons continue to attract significant research interest. Although solvated electrons are well known in radiation chemistry, the use of plasmas for their generation has not been widely explored due to their high reactivity and interfacial formation, which makes it exceedingly difficult to observe them. Indeed, their existence

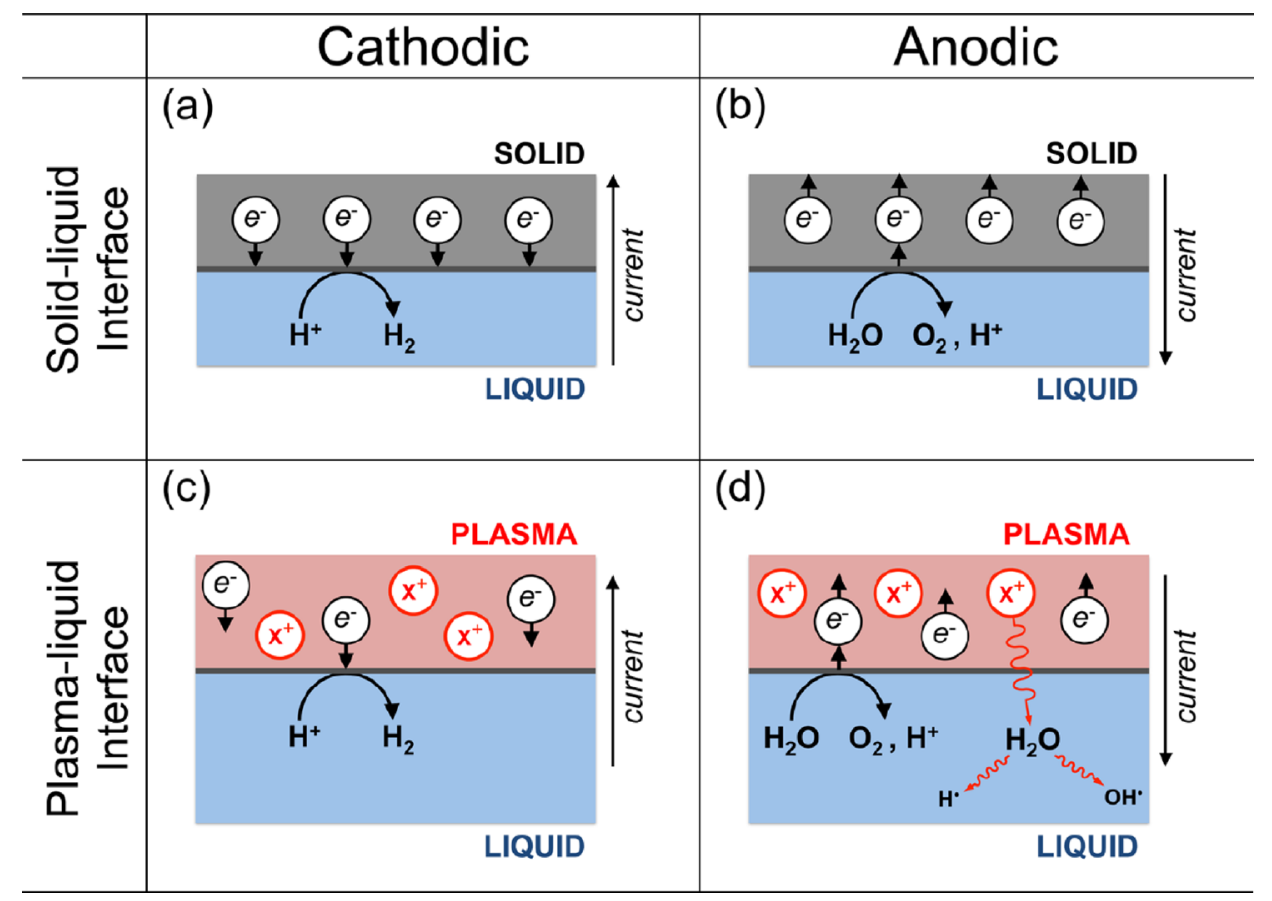

FIG. 13. Comparison of chargetransfer processes at solid-liquid and plasma-liquid interfaces. Both cathodic and anodic charge transfer, defined by the direction of current flow, are shown. The precursor cations undergo reduction to form thin films on the electrode surface. Anodic reactions release electrons from the electrode and oxidation of a species in the electrolyte. Reprinted with permission from R. Akolkar et al., J. Vac. Sci. Technol. A 31, 050811 (2013). Copyright 2013 AVS. 

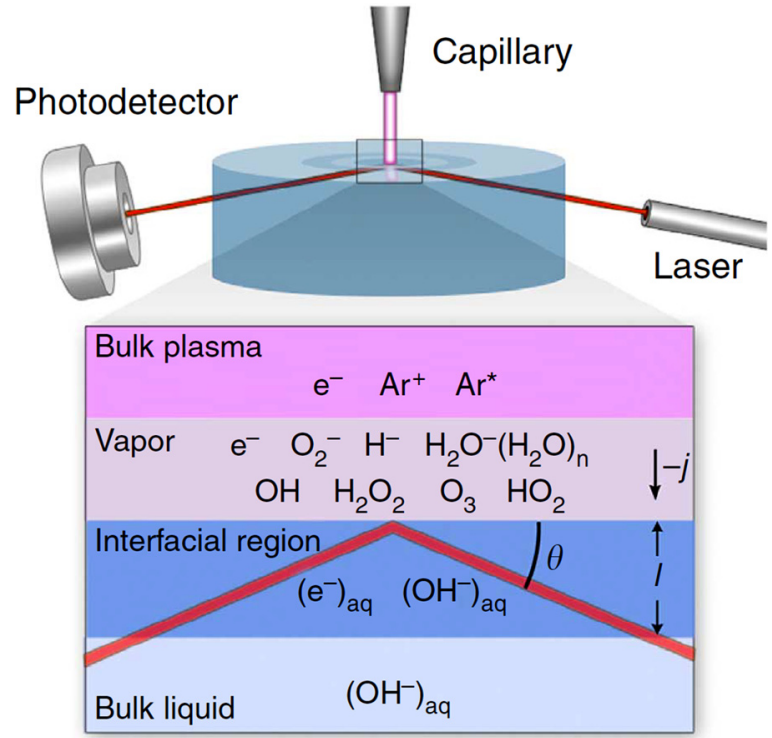

FIG. 14. Generation and detection of solvated electrons by an atmosphericpressure plasma. (a) Schematic of experimental apparatus for optical detection using a total internal reflection configuration. Anticipated chemical species in the different phases are also shown. Note: the submerged counterelectrode has been omitted from this figure to emphasize the optical measurement. Reprinted from P. Rumbach et al., Nat. Commun. 6, 7248 (2015). Copyright 2015 Springer Nature, licensed under the terms of the Creative Commons CC BY License.

has often been inferred from the scavenging reactions in the solution bulk rather than direct observation. ${ }^{139-141}$

\section{$\checkmark$ Plasma-liquid interface makes a difference-} Plasma electrons fully solvate in the bulk

Recent experimental evidence has shown that electrons generated at a plasma-water interface fully solvate prior to engaging in chemical reaction at a rate very similar to that of solvated electrons generated by pulse radiolysis. ${ }^{143}$ This was demonstrated using plasma formed at atmospheric pressure using argon as a processing gas. As shown in Fig. 14(a), a laser probed the plasma-water interface in a reflecting geometry to spectroscopically characterize the solvated electrons which have a lifetime corresponding to only a depth of $\sim 10 \mathrm{~nm}$. The formation of solvated electrons was confirmed by measuring their characteristic red absorbance.

This experiment showed that solvated electrons produced at the plasma-liquid interface were generated by a mechanism that is distinct from other approaches such as high energy radiation (e.g., X-ray, electron beam, etc.), offering the advantages of being simple and more economical. Furthermore, it may be more environmentally and human health-friendly, since it obviates the need for harmful radiation. Importantly, the resultant solution provides a highly reactive environment to study the behavior of the free electrons at the interface and near-surface regions. Indeed, the behavior of solvated electrons when subjected to the action of strong electric fields is not well-understood; however, evidence to date suggests an opportunity to control their concentration, spatio-temporal distribution, and penetration depth by controlling current density.

\section{The processes at biological tissues and biofilms interfacing the plasma}

The processes at the biological tissues and biofilms when in contact with relatively powerful plasma jets are of a critical importance for plasma-enabled medicine and biotechnology. These processes are relatively intense, with their application being protein denaturation via heating (about $50{ }^{\circ} \mathrm{C}$ ), as well relatively strong plasma effects and plasmadriven currents. ${ }^{144,145}$ Importantly, the processes (with the mass transfer, liquid heating, and generation of active species being the most important effects) at the plasma-tissue interface could be interpreted in the terms of plasma-liquid interaction. Similarly, a high degree of hydration of the microorganism biofilm (up to $85 \%-95 \%$ water) enables its modelling as a bulk liquid of semi-infinite depth. ${ }^{142}$ Figure 15 illustrates the widest spectrum of reactions in the framework of the reaction-penetration model for mass transfer

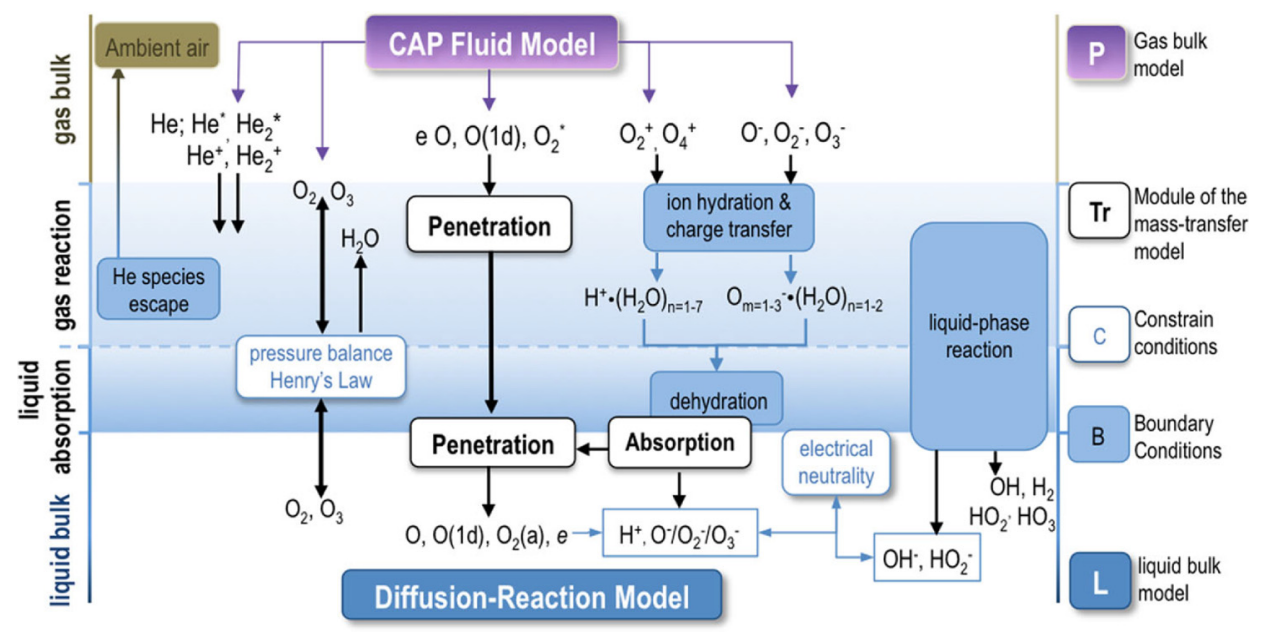

FIG. 15. The reaction-penetration model for mass transfer through the gas-liquid boundary, and its link to the fluid model for the gas bulk (marked with a purple box) and the diffusion-reaction model for the liquid bulk (for biofilm, marked with dark blue box). The mass-transfer model has a few components, marked with white box and black text. Constrain conditions for the balance of partial gas/vapor pressures and for the electrical neutrality between positive and negative ions are marked with white box and blue text; and hydration, other liquid-phase reactions, and dehydration are marked with light blue boxes. Reprinted with permission from C. Chen et al., Plasma Chem. Plasma Proc. 34, 403 (2014). ${ }^{142}$ Copyright 2008 Springer. 
through the gas-liquid boundary, related to the biological tissues and biofilms.

Importantly, for the bio-applications, the atmospheric plasma is sustained by highly energetic $(\sim \mathrm{few} \mathrm{eV})$ electrons. The biological effects of such plasmas are mediated by a wide range of highly reactive and moderately energetic species, e.g., chemical species in ground, excited, and metastable states, activated particles, and electromagnetic radiation $(\leq 6-8 \mathrm{eV})$. Where these species induce a range of chemical reactions in the cell, ${ }^{30,146}$ heat fluxes (with a gas temperature of $27-120^{\circ} \mathrm{C}$ ) and electric fields may apply an additional physical force on these biological targets. ${ }^{147}$

The transport of plasma agents from the interface into liquid is the most important process for the case being examined. In a relatively simple case, still considering the ongoing chemical reactions, the transport equation for the liquid bulk has the form:

$$
\frac{\partial C_{i, l}}{\partial t}=D_{i, l} \frac{\partial^{2} C_{i, l}}{\partial x^{2}}+\sum R_{i . l}
$$

where $C_{i, l}$ is the concentration of the solute, e.g., a plasmagenerated reactive species, $i, D_{i, l}$ is its diffusion coefficient in the solvent, e.g., water, and $R_{i, l}$ is the rate of chemical reactions characteristic of the solute $i$ in liquid $l{ }^{142}$

However, plasma at the interface with media that features micro- and nano-scale irregularities produces very strong electric fields, which cannot be neglected; ${ }^{148,149}$ in this case, the space-charge electric field which arises from the presence of all charged species within the solution will affect the transport of the plasma-generated species. In this case, the transport equation should consider the diffusion and drift terms, thus significantly complicating the problem $^{142,150}$

$$
\Gamma_{i, l}=\Gamma_{i, d i f f}+\Gamma_{i, d r i f t}=-D_{i, l} \nabla C_{i, l}+z_{i} \mu_{i, l} C_{i, l} F \vec{E} / N_{A},
$$

where $E$ is the electric field; $z_{i}$ and $\mu_{i}$ are the ion charge number and the mobility of the charged species, respectively; and $N_{A}$ and $F$ are the Avogadro and the Faraday constant, respectively. A self-consistent electric field could be found by solving the Poisson equation. ${ }^{151}$

Evidently, this system is too complex to be investigated experimentally in detail; moreover, at present, there are no means available to separate and study individually such a large number of interrelated reactions, caused and sustained by plasma, the electric field and current in liquids. In this case, modelling becomes the principal tool for revealing the key relations and factors, determining the effect of plasma and current onto tissues.

\section{Ultrasound effects in liquids}

While the exposure of liquids to powerful ultrasound energy, in general, results in the similar effects to that observed in laser and electrical breakdowns, i.e., producing the shock waves and high temperature spikes, it nevertheless has some distinguishing features and will be addressed in a separate section.
Extremely high pressure levels exceeding $1000 \mathrm{~atm}$ and predicted temperatures reaching $5000 \mathrm{~K}$ could be obtained in the collapsing acoustical bubbles, as illustrated in Fig. $16 .{ }^{152}$ In addition to powerful physical effects, the collapse leads to the generation of a range of reactive oxygen chemical species, e.g., hydroxyl radical and hydrogen peroxide, which drive sonochemical reactions. The nature and concentration of thus-formed chemical species are dependent on the pressure and temperature attained within the bubbles at the time of their collapse. From the chemical point of view, this system is also of a strong interest for the material treatment and activation, since tens of chemical reactions are possible inside a cavitation bubble. ${ }^{152,153}$

Even higher temperatures reaching $18000 \mathrm{~K}$ for the acoustic power of about $20 \mathrm{~W}$ and frequency of about $200 \mathrm{kHz}$ were obtained by numerical simulations, where the maximum pressure was estimated using the equation: ${ }^{154}$

$$
T_{\max }=T_{0}\left(\frac{R_{\max }}{R_{\min }}\right)^{3(\gamma-1)},
$$

where $T_{0}$ is the temperature of the solution; $\gamma$ is the specific heat ratio; and $R_{\max }$ and $R_{\min }$ are the maximum and the minimum radii of the bubble at the beginning and the end points of its collapse, respectively. Assuming that the liquid media is an incompressible fluid of infinite depth, the temporal dynamics of this spherical bubble is expressed using the Rayleigh-Plesset equation. ${ }^{152,157,158}$

$\checkmark$ Unprecedentedly strong conditions could be obtained in the ultrasound-created cavitation zone

Importantly, for the material treatment and activation applications, high temperature and pressure levels could be

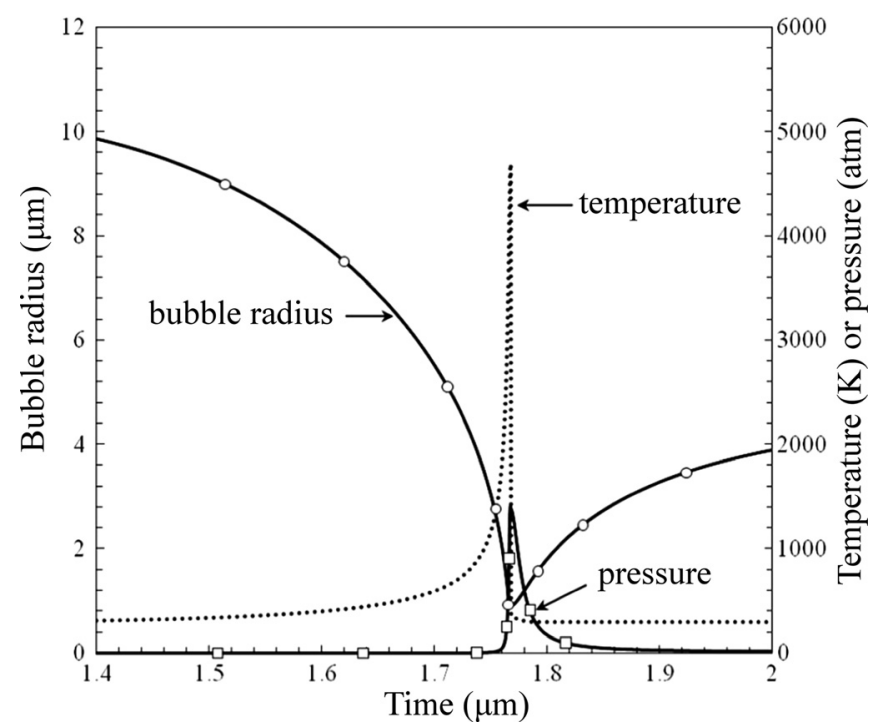

FIG. 16. Bubble radius and temperature and pressure inside a bubble as a function of time during the collapse phase of the bubble, for the conditions: ambient bubble radius $-3 \mu \mathrm{m}$, frequency $-500 \mathrm{kHz}$, acoustic amplitude$0.25 \mathrm{MPa}$, temperature-293 K, and static pressure - $0.1 \mathrm{MPa}$. The horizontal axis is only for $0.6 \mu \mathrm{s}$. A maximum bubble temperature of about $4600 \mathrm{~K}$ and pressure of about $1400 \mathrm{~atm}(140 \mathrm{MPa})$ are achieved at the end of the collapse. In this figure, we preferred to present the pressure inside a bubble in atm rather than MPa to only present it on the same axis (secondary axis) with the bubble temperature. Reprinted from S. Merouani et al., Ultrason. Sonochem. 21, 53-59 (2014). Copyright 2014 Elsevier. 
obtained by ultrasonic power in micro and nano-channels. ${ }^{66}$ This effect could not be achieved by electric breakdown. Even for microchannels of $100 \mu \mathrm{m}$, a temperature estimate of $3100 \mathrm{~K}$ was obtained. ${ }^{160}$ Application of microporous and nanoporous platforms in ultrasonicated liquid precursors is a promising approach enabling the synthesis of unique complex multi-material nanostructures such as metallic nanowires in nanoporous substrates. ${ }^{66,161}$

\section{E. Self-organization, patterns formation, and adaptive plasma at liquid interfaces}

Arising from an entropy minimum principle, selforganization is one of the principle drivers in the development of complex living and to some extent, non-living systems, where they acquire spatio-temporal or functional structures by means of intrinsic drivers. Albeit not to the same extent, self-organization plays a significant role in a number of physical systems, such as in the case of a glow discharge generated at atmospheric pressure, which forms self-organized stratified interface patterns.

Self-organizational processes at the gas-liquid interfaces were already mentioned in this work; here, we will address some exciting phenomena that relate to the plasma treatment and activation. Historically, plasma jet filamentation and self-organization into regular structures were discovered and observed many years ago. ${ }^{162-164}$

A representative example of the filamented organized structure obtained in high-pressure gas discharge is shown in Fig. 17(a), where the regular structure was obtained in helium discharge at the pressure of $122 \mathrm{hPa}$. Similar regular structures were obtained by simulation of self-organized growth of nanostructures from plasma [Figs. 17(b) and 17(c)].

Self-organization of regular structures on surfaces of liquids has many unique features and controls that are not available at the plasma-solid interfaces. Indeed, liquid surfaces are agile, they could easily and quickly change their physical and chemical characteristics, e.g., they could form a dense vapor atmosphere above the surface, and so on. As a result, the self-organized structures at the plasma-liquid interfaces feature impressive diversity and typology; they are movable, they can change easily in response to the change of the external conditions (e.g., pressure, temperature, composition, and many more), and what is most important, the selforganized structures at the plasma-liquid interfaces are selfadaptive; this means that the plasma pattern and plasma parameters adjust to the changes in the system, thus acquiring novel, unique features. ${ }^{30,115}$ Figure 18 illustrates the typical anode patterns with different water electrical conductivities. Importantly, self-organization (filamentation) requires a certain threshold current density, and cannot be reached at too low current levels.

$\checkmark$ Relatively strong current and relatively intense plasma are required to demonstrate self-organization

Due to the above described advantages, as well as the ease with which such plasma can be ignited and sustained at atmospheric pressure, plasmas with self-organized stratified interface pattern have been attracting interest for a range of applications. ${ }^{165}$ Figure 19(a) depicts a representative experimental plasma generation setup which has been used to generate self-organized stratified patterns at the interface between the plasma and the liquid with high degree of definition. In this particular configuration, an anode is a thin foil of copper, which is fully immersed into the deionized water. A tungsten cathode is suspended above the surface of the liquid, and the discharge is ignited by applying the voltage across these electrodes. Figure 19(b) depicts optical images of a variety of self-organized stratified patterns that could be attained using this system, including shapes of notable complexity that consist of confocal and radial lines of varying density, and structures with some degree of axial and radial symmetry. Figure 19(c) shows the corresponding $I-V$ characteristics for a wide number of discharge patterns, suggesting that the entire spectrum of $I-V$ characteristics can be split into four distinct stages: (I) low voltage and current, where a single-filament contracted discharge is produced; (II) the current and temperature increase, resulting in a heat radiation-supported discharge; (III) an intermediate stage, where the unstable discharge alternates between that
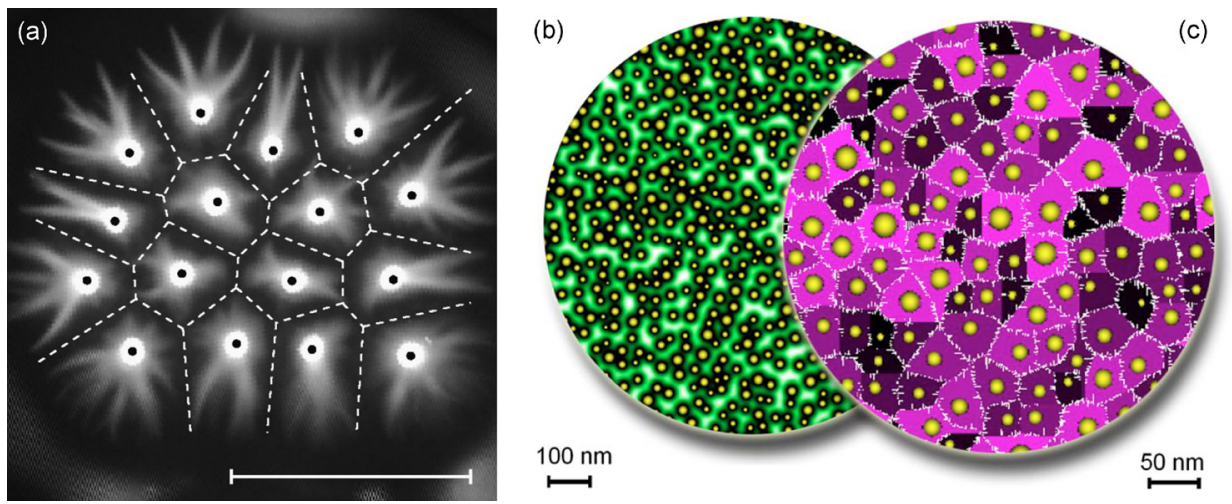

FIG. 17. (a) Discharge with predefined filament locations. White broken lines and black points on filaments represent the edges of the Voronoi diagram and the set of reference points, respectively. Gas gap is $3.0 \mathrm{~mm}$ of which $1 \mathrm{~mm}$ is the perforated insulating film defining the reference points. Glass plates have thicknesses of 0.7 and $1.0 \mathrm{~mm}$. Pressure of nitrogen is $122 \mathrm{hPa}$. Reprinted with permission from Appl. Phys. Lett. 81, 3338 (2002). ${ }^{155}$ Copyright 2002 AIP Publishing LLC. (b) and (c) Fragments of the simulation domain with the calculated adatom density field and calculated flux distribution diagram pattern [a kind of enhanced Voronoi diagram shown in (a)]. Nanodots are shown as small goldish semispheres. Reprinted with permission from Appl. Phys. Lett. 95, 243102 (2009). ${ }^{156}$ Copyright 2009 AIP Publishing LLC. 

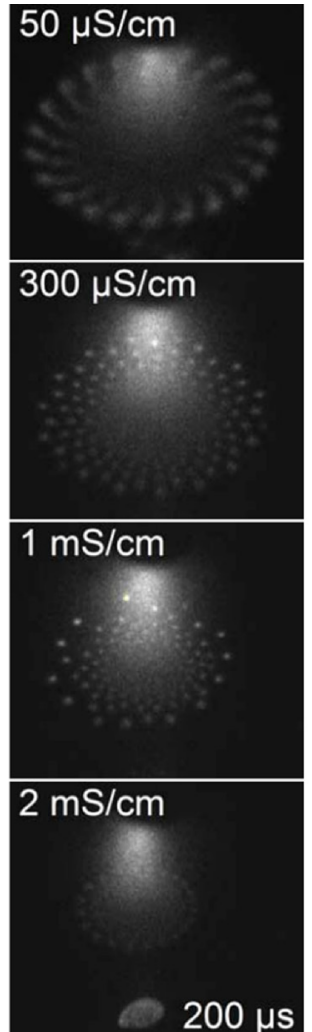
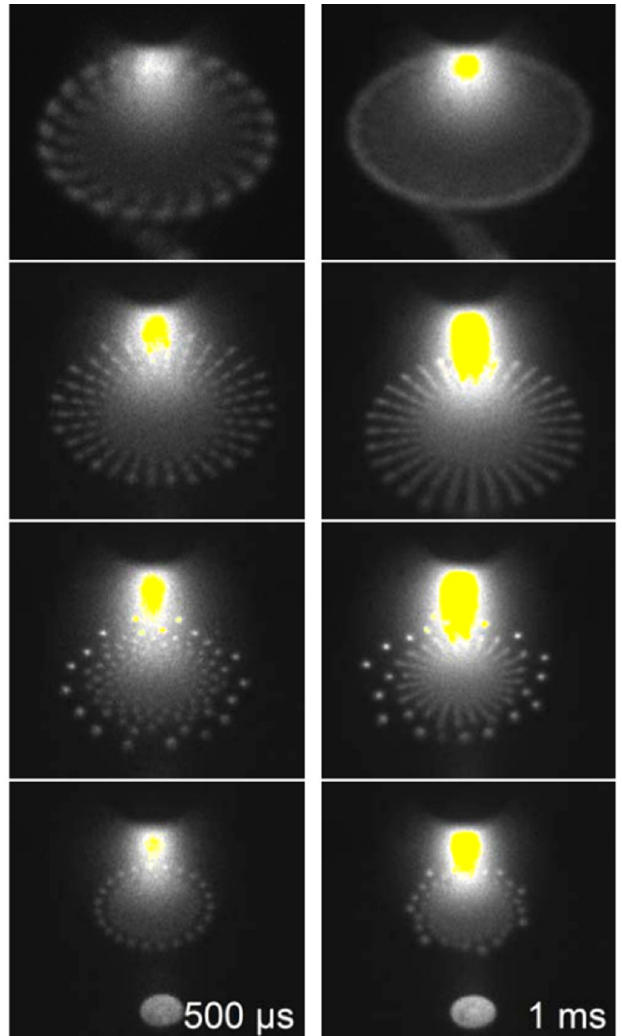
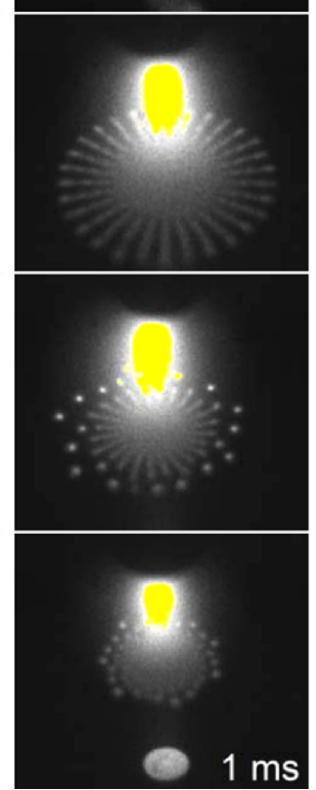
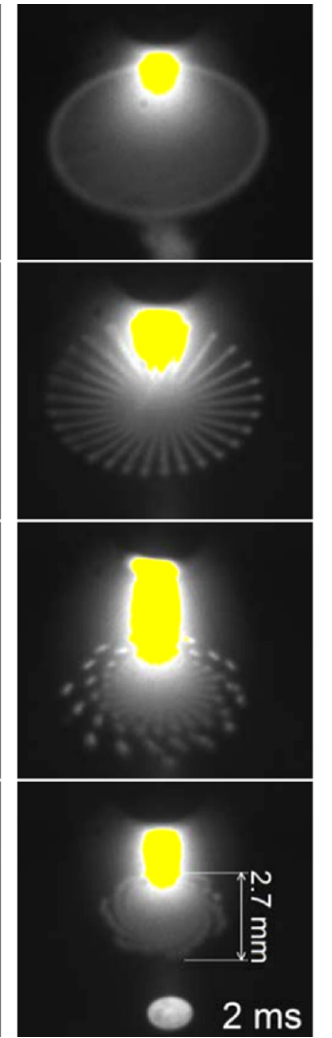

FIG. 18. Overview of the anode patterns with different water electrical conductivities at $70 \mathrm{~mA}$. The exposure time and the water electrical conductivity are indicated in the figure. The images have the same gain for the same exposure times. Reprinted from J. Appl. Phys. 105, 083312 (2009). Copyright 2009 AIP Publishing LLC. ${ }^{159}$ produced under stages (II) and (IV); (IV) at the highest current, a stable multi-filament discharge is produced and organized into a wide variety of patterns.

The ability of a plasma to self-adapt when it interfaces the liquid is one of the most prominent properties of this system. ${ }^{116}$ While self-organization affects mostly the plasma stream structure, shape, and other similar characteristics in response to the external conditions (such as property of the liquid and of the environment), self-adaptation is a chain of processes that involve plasma-driven alterations of the liquid (chemical composition, temperature, electrical conductivity, $\mathrm{pH}$ and many more) and then change of the plasma parameters in response to

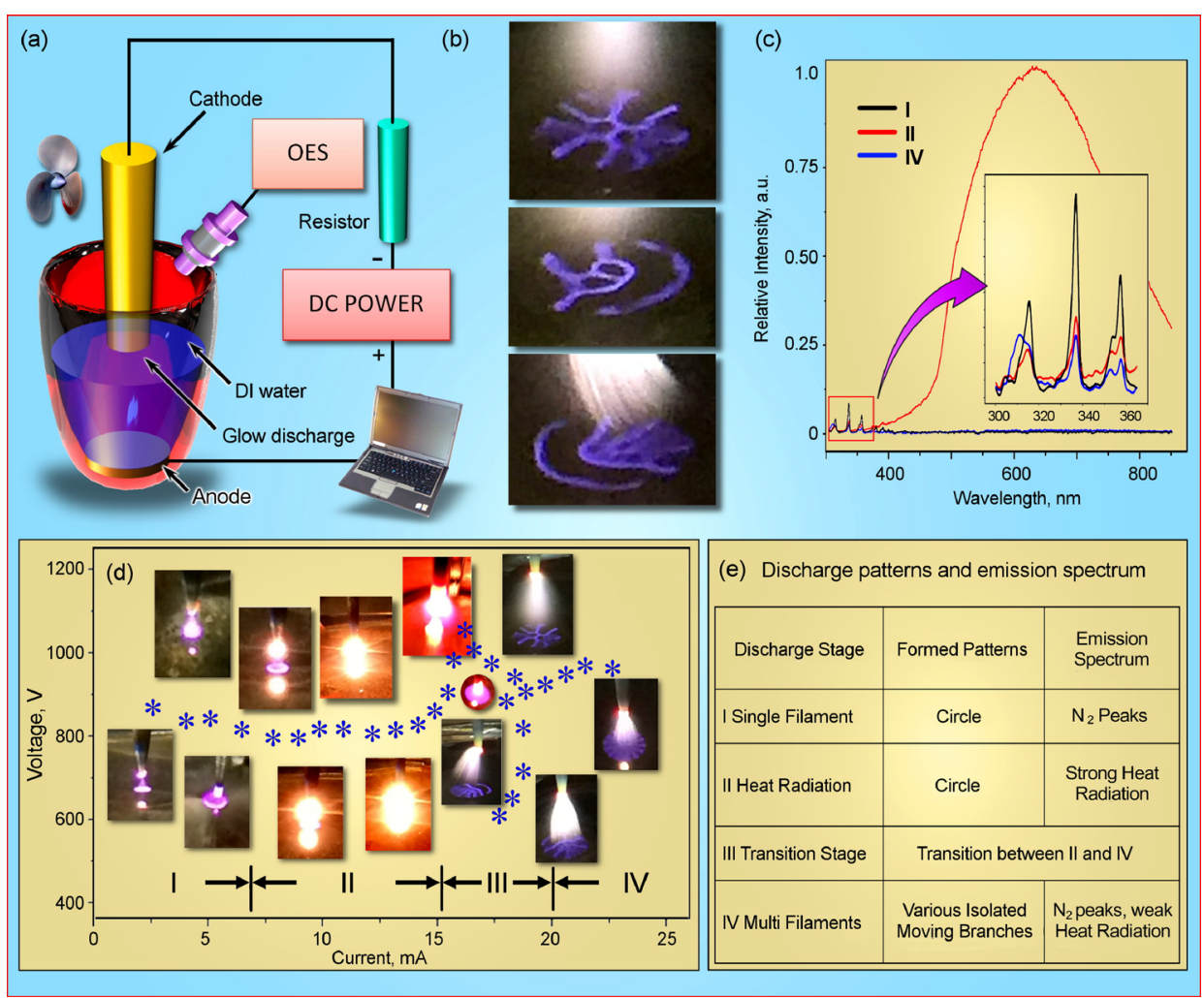

FIG. 19. (a) Schematic representation of the atmospheric pressure glow discharge setup. A small (several $\mathrm{mm}$ ) gap between the cathode and surface of liquid accommodated a bunch of plasma. (b) Optical photographs of the discharge patterns above the therapeutic media during the activation process. The self-organized patterns have complex structure strongly depending on the voltage-current conditions. (c) Optical emission spectrum by the atmospheric pressure glow discharge above DI water taken using UV-visible-NIR in the $200-850 \mathrm{~nm}$ wavelength range. (d) Current-voltage dependence of the system with optical photographs of the self-organized stratified interface patterns. Four discharge stages could be clearly determined. (e) A table summarizing the relations between discharge stages, selforganized patterns, and optical emission. Notably, no self-organization and filamentation could be detected at stage I, where current is too low. Reprinted from Z. Chen et al., Sci. Rep. 7, 12163 (2017). Copyright 2009 Nature Publishing. ${ }^{30}$ 
(A)

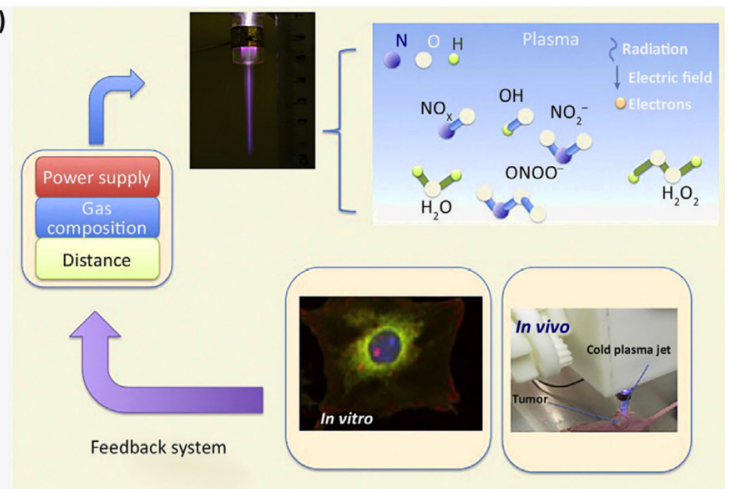

(B) (a)

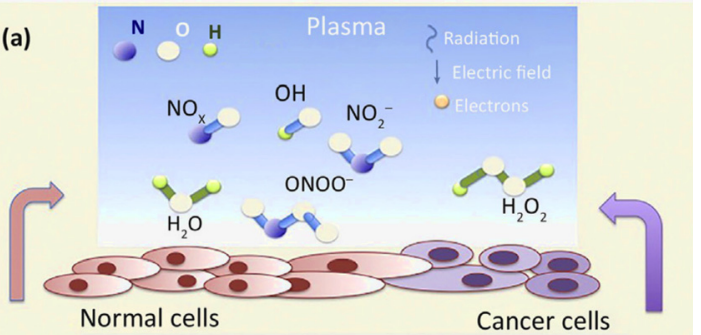

(b)
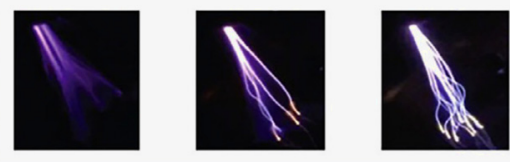

FIG. 20. Actively adaptive plasma. (A) Concept of adaptive plasma. Cold plasma treatment in vivo or in vitro will trigger differing responses in cancer cells and normal cells. The signal based on these responses will be measured, recorded, and analyzed by a plasma system. Through the feedback system, the chemical composition will be modulated through changes in the power supply, the gas composition, and the distance between the plasma source and the cells. (B) Plasma self-adaptation-based self-organization and pattern formation. Cancer cells and normal cells exhibit significant differences in membrane potential, which can be used as a signal to trigger plasma self-organization. As a result, cold plasma is affected differently by normal and cancer cells (a). The distinct response of plasma to cells and tissue leads to plasma mode transition (b). Reprinted from M. Keidar et al., Trends Biotechnol. (in press). Copyright 2017 Elsevier. ${ }^{116}$

those alterations; next, the plasma changes its properties in response to the liquid parameters. The self-adaptation process proceeds until a new, stable state is reached. A comprehensive review on the physical mechanisms and processes leading to self-organization at the stratified plasma-surface interface can be found elsewhere. ${ }^{166}$

Thus, the adaptive plasma is able to adjust its composition to reach the optimal state through the interaction with the alternating environment. Next stage would be the actively adaptive plasma system (see the illustration of this concept in Fig. 20). This example is based on the use of cold atmospheric pressure plasma for the treatment of biological tissues, specifically inhibition of cancer cells; the choice is due to high complexity and cost of the first conceptual experiments and importance of cancer fighting problem.

This approach relies on the live feedback loop, whereby the biological responses of the plasma treated living organism are detected, and the plasma is adjusted accordingly in terms of its intensity and chemical composition to deliver the necessary therapeutic benefit, e.g., selective death of cancer cells. Besides, the control over the generation of reactive species or the interaction potential between plasmas and living targets could be achieved. Figure 20(b) depicts the central concept of how plasma interactions with cells can lead to transitions between different plasma discharge modes, thus potentially enabling plasma self-adaptation in a sophisticated, non-linear mode. ${ }^{167}$

$\checkmark$ Introduction of an active feedback into the system with a plasma-liquid interface ensures conceptually new level of adaptation, with a set of experimentally proven, unique results

\section{F. Synergistic effects in energy-exposed liquids}

To illustrate some promising capabilities of synergistic effects for the nano- and material fabrication under exposure of several types of energy, we will examine combinations 2 (laser plus ultrasound) and 4 (radiolysis) (see Table II). As shown below, synergistic effects could produce unique structure or result in an extremely high efficiency of the process or could ensure other useful features. ${ }^{37}$ Applications in medicine for anti-cancer therapy ${ }^{168-170}$ and fabrication of drug loaded core-shell nanoparticles to inhibit cancer, ${ }^{171}$ for antibacterial treatment using synergistic reactions between, e.g., silver nanoparticles with reagents, ${ }^{172}$ and for the fabrication of various nanoparticles and complex nanosystes ${ }^{39,173}$ are among the characteristic examples.
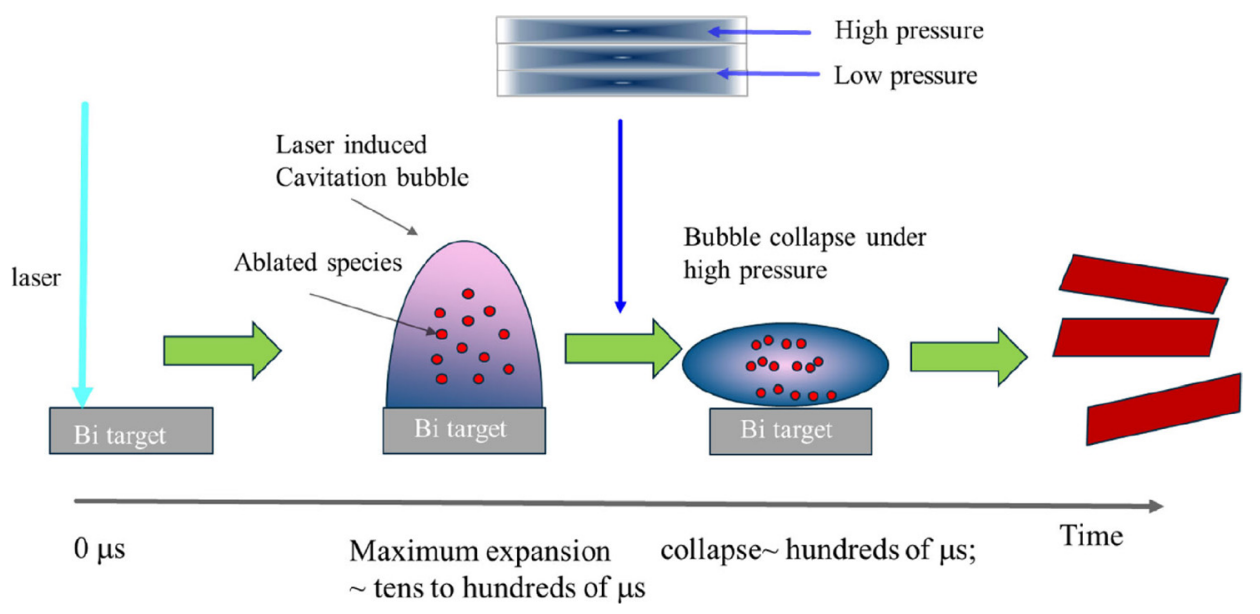

FIG. 21. Schematic representation of the proposed synergy-enabled nanosheets growth mechanism. Generation of cavitation bubble by laser irradiation and the following collapse causes unique conditions that cannot be ensured by the action of a single type of energy. As a result, bismuth-based nanosheets were produced. Reprinted with permission from L. EscobarAlarcon et al., Appl. Phys. A 122, 433 (2016).$^{94}$ Copyright 2016 Springer. 


\section{$\checkmark$ Synergistic effects are particularly strong and effi- cient at liquid interfaces}

Apparently, synergistic effects appear when more than one type of energy interfaces the liquid. Laser ablation of solids in solution has been recognized as a promising approach for the generation of nanoscale materials with a wide range of possible shapes and sizes, including fullerenes, nanoscale spheres, tubes, and rods (see references in Table I and herein). Sonochemistry is now a well-established technology, but apparently, several shortcomings are intrinsic to this method; most remarkable feature is inappropriateness for an efficient production of the precursor material capable of free-levitating in the liquid media.

The idea of obtaining a synergistic effect by application of laser ablation of a solid target to produce reagents in the liquid, and utilization of highly powerful cavitation effect caused by the intense ultrasonic power has been quite fruitful. Ultrasound-aided liquid laser ablation was successfully used to synthesis nanosheets of bismuth(III) oxide, in which case the use of the ultrasound field promoted the self-assembly of nanosheets, whereas in the absence of the ultrasound field, the process yielded primarily nanoparticles. Figure 21 provides possible mechanisms for the origin of the nanosheets. There is a strong potential to concomitantly apply these processes and arising extreme temperature and pressure conditions for the synthesis of other 2D-ordered nanostructures. It should be noted that $\mathrm{Bi}$ nanosheets were recently synthesized in the authors' lab (Belmonte et al.) via quite a different process, namely, by electric discharges in liquid nitrogen; thus, other mechanisms different from those described above may provide an efficient alternative for the self-assembly of nanosheets.

Radiolysis, i.e., application of ionizing radiation to dissociate and ionize molecules and atom in liquid, could also be used in combination with other electric-current based techniques such as electrochemistry, resulting in quite useful effects. As it was shown in Table II, this method was successfully applied to produce and modify various nanostructures such as nanotubes arrays, nanoparticles, and bimetallic nanoparticles by gamma-assisted radiolytic reduction via electrochemical anodization. Figure 22 illustrates the physical mechanisms involved in the transformation and selfassembly of core-shell nanoparticles in radiolytic reduction via electrochemical anodization.

Apparently, quite different functions of gamma-radiation and electrochemical anodization in this process ensure pronounced synergistic effects. Under a moderately low dose of gamma radiation, noble metal ions are first reduced to form the inner core of the core-shell structure. The noble ions are preferentially reduced because of the fast electron transfer to them from their less noble counterparts.

\section{APPLICATIONS OF ENERGY-DRIVEN PROCESSES IN LIQUIDS}

The above outlined effects and processes in liquids and at liquid interfaces under the action of various kinds of energy have demonstrated a very attractive, fundamental potential of this techniques for various applications. Here, we will outline some examples of successful applications of the processes in liquids and at gas-liquid interfaces for the material treatment and activation, with the main aim to illustrate the widest diversity and universal capabilities of this approach, which ensures strikingly different outcomes ranging from nanosynthesis to biological and medical applications.

\section{A. Additive processes in liquids: Nucleation and growth}

Here, we will start from the most common and promising application of processes in liquids, namely, the nucleation and growth of nano- and microstructures, from simplest nanodots to extremely complex hierarchical and multilayer metamaterials. ${ }^{174}$

Nucleation is the first and often the key stage of the structure formation from gas or liquid phases. Film structure, quantum size pattern density, chirality of nanotubes, and many other critical parameters will be governed by the initial pattern and structure of nuclei, i.e., very small (near-critical) embryo. The number density of nucleus embryo is defined largely by the concentration of elemental particles (ions, atoms, and radicals) that form the nucleus. In general case, the critical (i.e., the smallest stable) size is defined by the competition of surface free energy and bulk Gibbs free energy of embryo (Fig. 23). Once the critical embryo dimensions are reached, the conditions become conducive to the growth of the grain. On the other hand, when the dimensions of the grain are below the critical size, spontaneous annihilation of the embryo takes place owing to high surface free energy. For example, in the radio-frequency argon microplasma sustained on an aqueous solution of ethyl alcohol, carbon nanoparticle and nanotubes were synthesized with

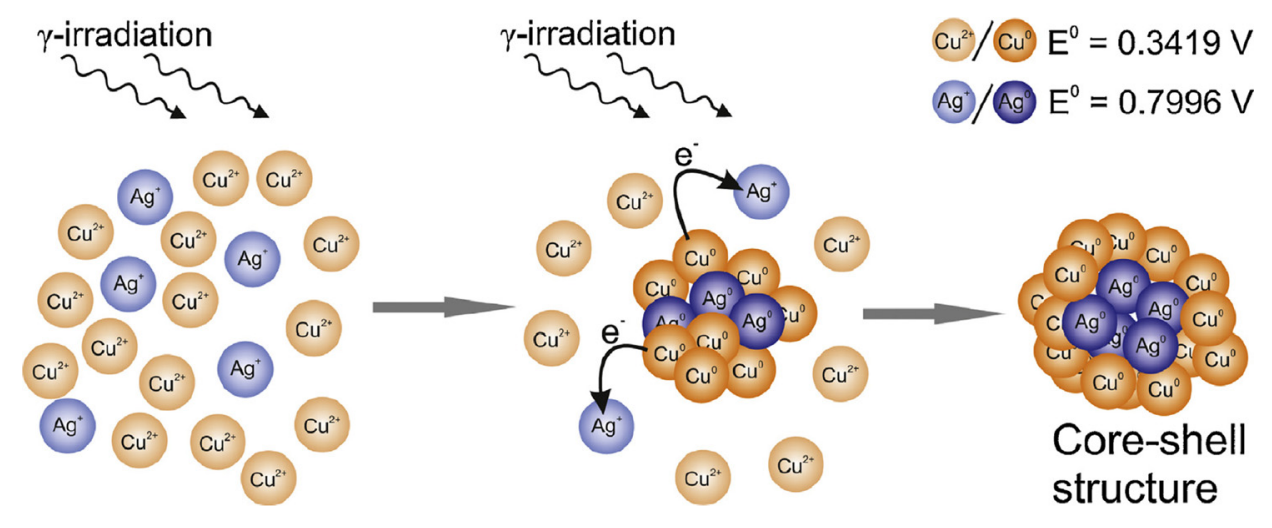

FIG. 22. Basic physical processes involved in the formation of bimetallic nanoparticles by gamma-assisted radiolytic reduction via electrochemical anodization. Reprinted with permission from M. Nischk et al., Appl. Surf. Sci. 387, 89 (2016).$^{99}$ Copyright 2016 Elsevier. 


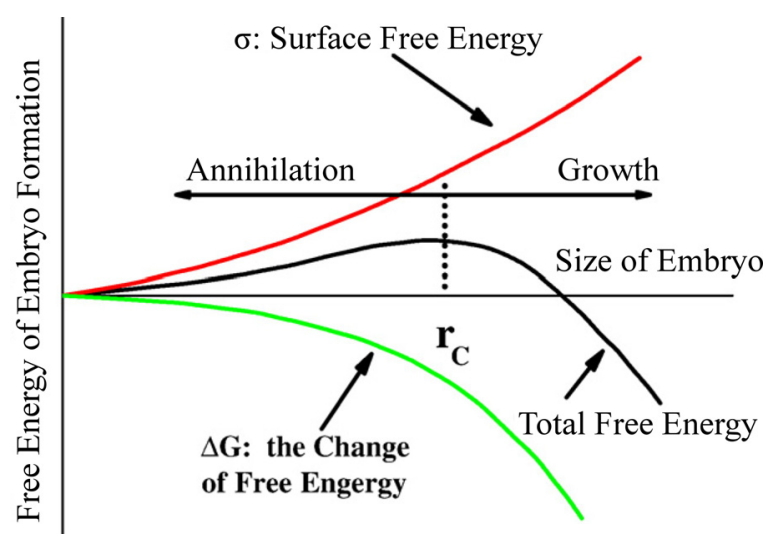

FIG. 23. Schematic of the correlation between the free energy and the size for the embryo formation. When $r<r_{C}$, the embryo is annihilated because of high surface energy of embryo. On the other hand, the embryo growth is dominant at $r<r_{C}$. Reprinted with permission from Q. Chen et al., Thin Solid Films 516, 4435 (2008). ${ }^{175}$ Copyright 2008 Elsevier.

$\mathrm{CH}$ and $\mathrm{C}=\mathrm{O}$ complexes at a concentration above $5 \% .{ }^{175}$ The abundance of the reactant determined both the size and the concentration of the nanoparticles, where both were found to increase as the reactant concentration exceeded $10 \%$. Since most of the plasma-forming excited species are consumed for the decomposition and activation of neutral precursors in the solution, the plasma should be intense enough to produce the supercritical density of active reactants capable to sustain continuous nucleation in the solution. ${ }^{176}$

$\checkmark$ Plasma and plasma-generated electric current densities, i.e., power density in liquid precursor, are the triggers that overcome thresholds of nucleation

How does the growth of complex structures proceeds in liquids under the action of electric current or other energy fluxes that ensure ionization, dissociation, and other types of reactant activation? The key mechanisms that manifest in all of these processes can be illustrated using an example of a discharge-driven electrolytic oxidation with alternating pulsed unipolar and bipolar regimes. When the oxidation takes place in a predominantly energetic penetrating discharge, a lower number of anions from the aluminate electrolyte are incorporated into the resulting thin film, the structure of which is pancake-like, with large internal pores. Indeed, the latter structure is common for solutions that contain a low or moderate concentration of electrolyte, in the absence of a negative pulse. Using a solution with a higher concentration of $\mathrm{NaAlO}_{2}$ enabled the rapid deposition of thin films with a significantly greater integration of anions under sintering arc and soft sparking conditions.

The mechanism of deposition of films with large micropores, e.g., at low concentration of the electrolyte, is supported by the experimental observation that every individual spark is encapsulated in a nonluminous gas bubble, ${ }^{177}$ on the surface of which electrolyte anions may be located. The flow of electrons from the solution to the gas bubble initiates the discharge. However, it should be noted that free electrons may present themselves initially at the interface between the oxide and the solution irrespective of the presence of the gas bubble. High pressure and temperature within the discharge channel drive the chemical reactions between the chemical

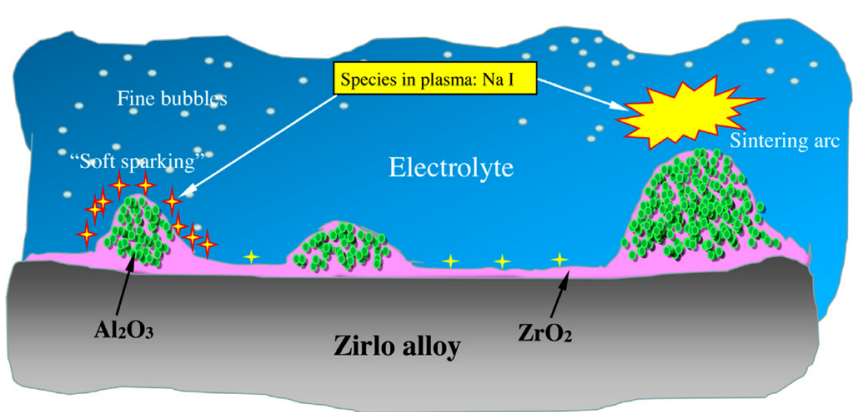

FIG. 24. Coating formation in $\mathrm{NaAlO}_{2}$ under the unipolar regime. Reprinted with permission from Y. Cheng et al., Electrochim. Acta 225, 47 (2017). ${ }^{44}$ Copyright 2017 Elsevier.

species in the electrolyte and the solid surface of the substrate, which lead to formation of new oxides.

In contrast, application of more concentrated electrolytes and the addition of a negative pulse have led to the formation of a film with higher level of homogeneity and greater thickness, and a significantly higher concentration of $\mathrm{Al}$ species. The negative pulse is thought to promote the formation of a soft sparking discharge, ${ }^{178}$ typically linked to more rapid deposition of electrolyte species; a schematic of the process illustrating the soft sparkling and sintering arcs involved in the deposition is shown in Fig. $24 .^{44}$

$\checkmark$ Spark under water triggers the formation of dense, quality structures

Very complex multilayer structures of the type of alternating layer-by-layer mesoporous structures could be formed in similar processes (Fig. 25). Many future technologies, e.g., advanced electrocatalysis and thin film devices rely on material structures composed of alternating thin films of different metals owing to the enhanced performance characteristics that such materials can deliver. The synthesis of such structures using conventional methods, e.g., atomic layer deposition, is time consuming. On the other hand, such mesoporous structures can be formed using a combination

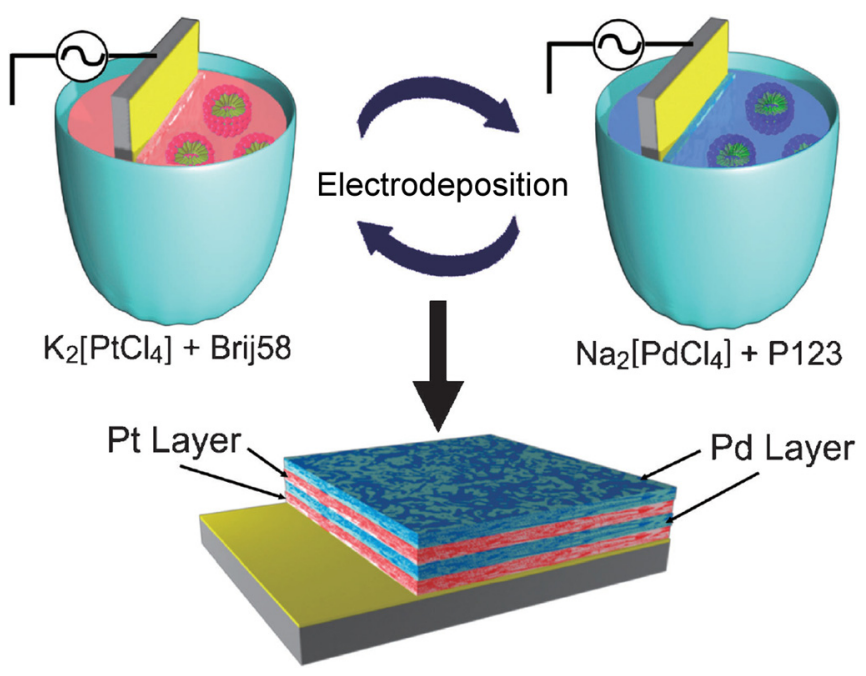

FIG. 25. Electrochemical micelle assembly for films consisting of alternating layer-by-layer mesoporous platinum and palladium structures. Reprinted with permission from K. Ariga et al., Chem. Lett. 43, 36 (2014). Copyright 2014 The Chemical Society of Japan. ${ }^{179,180}$ 
approach, where micelle assembly in a dilute surfactant electrolyte is integrated with advanced layer-by-layer deposition. This approach allows for fabrication of complex multilayer hybrid thin films efficiently, as demonstrated by the fabrication of alternating platinum and palladium structures with well-defined mesopores. ${ }^{179}$

Formation of bi-metallic mesoporous gold-platinum structure provides a good illustration for this technique. ${ }^{181}$ Specifically, the formation of the structure is initiated with the formation of $\mathrm{Au}$ seeds following the reduction of $\mathrm{Au}$ ions, which occurs much faster compared to $\mathrm{Pt}$ due to the significant difference in the reduction potentials of these two metallic species. Next, the Au seeds become rapidly overgrown by $\mathrm{Pt}$ shells, where their dendritic nanostructure is attributed to the presence of non-ionic surfactant. In addition to AuPt core-shell structures, AgPt, PdPt, and AuPdPt coreshell structures have been successfully produced using this approach. One of the key benefits of this method lies in the reduction of the quantity of expensive and scarce Pt needed for the synthesis. The method is amenable to fabricating of a broad variety of structures of various chemical make-up, structure, and functionality. ${ }^{181}$

\section{$\checkmark$ Traditionally used for simple coatings, electrolysis is capable of forming quite complex structures}

\section{B. Additive processes at plasma-liquid interfaces}

While electric current is capable of producing sparks and sintering arcs directly in liquids, and could be used for the fabrication of various structures and complex material systems, the direct effect of plasma discharge onto the liquid surface presents itself as another attractive scheme for the sophisticated and highly controllable method for nanofabrication. As a characteristic example, the fabrication of "ideal" metallic (gold and silver) nanoparticles by microplasmas interfacing the surface of liquid precursor could be discussed.

In this technique, a stable plasma discharge with a current of $\approx 1-10 \mathrm{~mA}$ is generated and sustained by applying a $\mathrm{dc}$ power. The discharge initiates a series of reductionoxidation reactions in the solution, leading to particle nucleation and subsequently, growth. The oxidation of the metal foil immersed in the electrolyte via electric current-driven oxidation leads to liberation of positively charged metal ions into the solution. ${ }^{182}$ The $\mathrm{pH}$ of the solution and current density determines the rate of metal dissolution, with mildly acidic solutions and greater current densities generally associated with higher dissolution rates. This is followed by the migration of the positively charged metal ions in the direction of the cathode, driven by the potential gradient of the solution. Upon reaching the cathode, which is determined by the microplasma-liquid interface, metal ions are reduced to the oxidation state of zero by gaining electron, the latter being generated in the gas phase and accelerated towards the surface of the solution. The process allows for free nucleation, growth, and dispersion of metal particles within the solution, with stabilizers, such as fructose, used to prevent unwanted clustering of the particles by binding to their surface. ${ }^{183}$
Figure 26(a) shows the high quality and uniformity of spherical crystalline silver nanoparticles produced using this approach. Transmission electron microscopy of these $10 \mathrm{~nm}$ particles indicate a lattice spacing of $0.20 \mathrm{~nm}$, corresponding to the 200 crystalline plane of face-centered cubic Ag. Au particles of similar quality and size prepared using the same technique are shown in Fig. 26(b). When the microplasma is used to reduce $\mathrm{AgNO}_{3}$ in water, thus-produced $10 \mathrm{~nm} \mathrm{Ag}$ particles are also crystalline, as depicted in Fig. 26(c), whereas the reduction of $\mathrm{HAuCl}_{4}$ solution results in $10 \mathrm{~nm}$ crystalline $\mathrm{Au}$ particles, as shown in Fig. 26(d). These examples highlight the flexibility of this process. Complex structures could also be prepared using this approach; for example, high-quality platinum nanoparticles and carbon-supported platinum nanoparticles $^{54}$ can be produced through plasma reduction of $\mathrm{H}_{2} \mathrm{PtCl}_{6}$ and carbon powder solutions. ${ }^{184}$

A quite different type of plasma, namely, the atmospheric-pressure radio-frequency (RF) plasma jet interfacing the acid solution, could be used for the production of perfectly crystalline metallic nanoparticles. In these experiments, the two principal set-ups were investigated, differing in the orientation of the anode (i.e., horizontal vs vertical) and the distance separating the surface of the electrode and the plasma jet (Fig. 27, left panel). The discharge was generated upon application of a direct current voltage $(20-40 \mathrm{~V})$ between the plasma-generating electrode and the anode. It should be noted that Fig. 27 shows a very common configuration for the atmospheric-pressure plasma jet, which includes a grounded and a powered electrodes, the latter
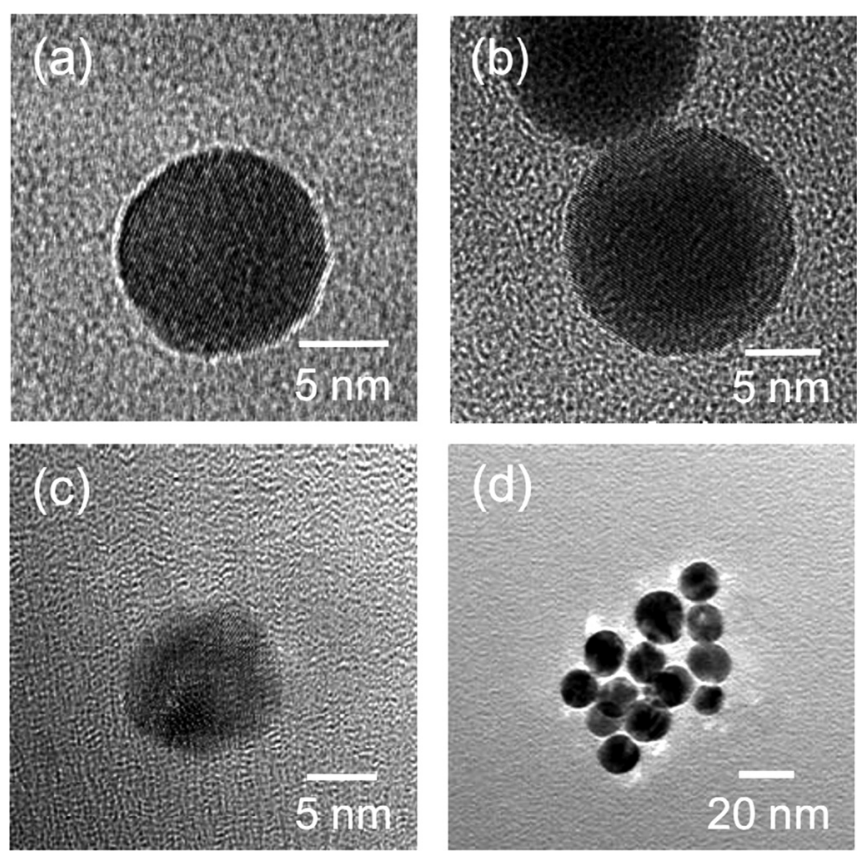

FIG. 26. TEM images of (a) Ag nanoparticles synthesized from anodic dissolution of $\mathrm{Ag}$ foil and microplasma reduction (process time $=10 \mathrm{~min}$ ), (b) $\mathrm{Au}$ nanoparticles synthesized from anodic dissolution of $\mathrm{Au}$ foil and microplasma reduction (process time $=30 \mathrm{~min}$ ), (c) Ag nanoparticles synthesized from microplasma reduction of aqueous $\mathrm{AgNO}_{3}$ solution (process time $=10 \mathrm{~min}$ ), and (d) Au nanoparticles synthesized from microplasma reduction of aqueous $\mathrm{HAuCl}_{4}$ (process time $=10 \mathrm{~min}$ ). Reprinted with permission from Appl. Phys. Lett. 93, 131501 (2008). Copyright 2008 AIP Publishing LLC. 
(a)

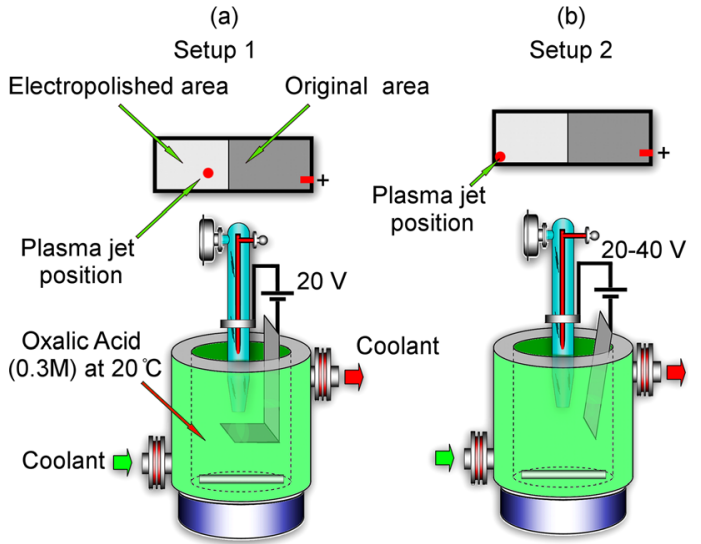

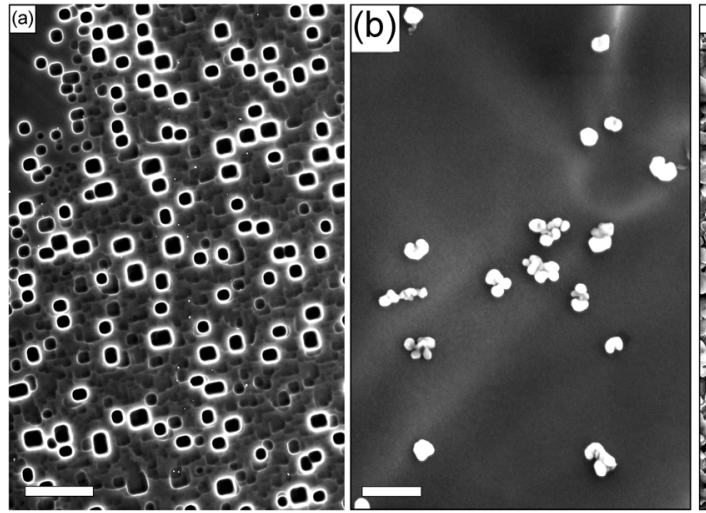

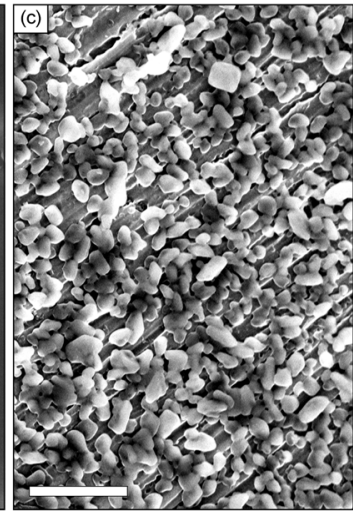

FIG. 27. Left panel: Two main configurations (setup 1 and setup 2) used for the atmospheric plasma-enhanced anodization and nanoparticle fabrication. In the first scheme, (a) the distance between the plasma jet and the electrode is $10 \mathrm{~mm}$. In the second scheme (b), the distance is $2-3 \mathrm{~mm}$. Right panel: SEM images of the holes on plasma-anodized aluminum surface (a) and alumina nanoparticles fabricated by the atmospheric plasma jet treatment in oxalic acid for $0.5 \mathrm{~h}$ (b) and by spark mode (c). Scale bars are $2 \mu \mathrm{m}, 200 \mathrm{~nm}$, and $1 \mu \mathrm{m}$, respectively. Reprinted with permission from J. Fang et al., IEEE Trans. Plasma Sci. 43, 765 (2015). Copyright 2015 IEEE.

encased in an insulating tube-form body. In this example, the power is generated using an RF supply and applied using a RF step-up transformer to maximize the power transfer.

The generation of an arc is mitigated by the use of a limiting resistor, which effectively limits the total discharge current. The processing gas used to sustain the plasma, typically Ar or He, is supplied at a flow rate of up to $1000 \mathrm{sccm}$.

Figure 27(a) (right panel) shows the morphology of the structure that develops at the fringes of the plasma-metal contact point, indicating lower level of porosity and a more regular rectangle-like shape of the pores, which were $\sim 1 \mu \mathrm{m}$ in dimension. The relatively low level of porosity suggests that although the structure is similar to that of foam, not all pores may be interconnected. Figure 27(b) (right panel) shows a representative depiction of the alumina nanoparticles located beyond the edges of the point where plasma comes into contact with the metal. Electron microscopy reveals the crystalline structure of thus-formed alumina nanoparticles, which are $\sim 50-100 \mathrm{~nm}$ in dimension. The propensity of these nanoparticles to agglomerate may be related to the effects of the electrical forces that may arise during the processing. Generation of sparks between the plasma and the surface of the solution (i.e., partial sparking) leads to the synthesis of 200-300 nm particles which cover the surface of the electrode with continuity similar to that of a film [Fig. 27(c)]. Unlike other particles, thus-formed particles do not clearly display crystalline facets, potentially as a result of localized melting brought about by rapid temperature increases in the micro-arcs. The surface of the electrode beneath the layer of the particles does not show any damage, suggesting that the particles were synthesized in arc spots and subsequently transferred to Al surface, driven by the acid flux and plasma stream. ${ }^{56}$

$\checkmark$ Current density in liquid strongly influences the result, and the sparking essentially changes the outcome

Figure 28 illustrates one more example of the efficient application of additive nanofabrication at plasma-liquid interfaces. In this case, the plasma-assisted electrolysis was used to produce nanoscale magnetite, i.e., nanosized magnetic particles which could find many useful applications. In this technique, plasma performs the role of an electrode. In addition, plasma acts as a source of free electrons or ions, which are delivered directly into the solution, triggering chemical reactions that may not necessarily occur under

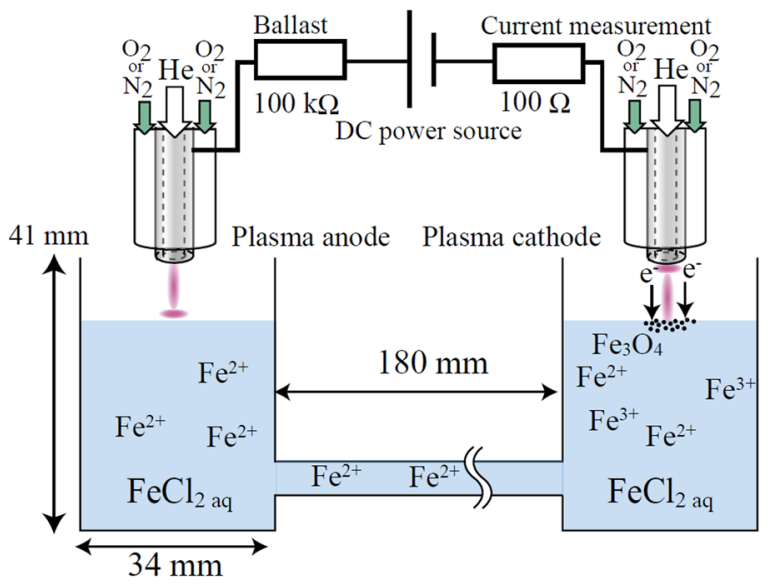

(a)

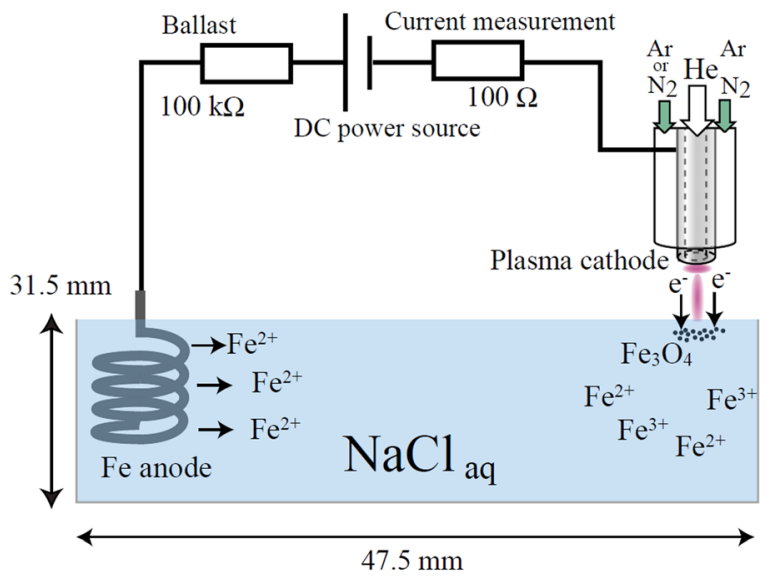

(b)

FIG. 28. Experimental setup for synthesis of magnetite using plasmaassisted electrolysis. (a) $\mathrm{Fe} 2+$ is supplied from $\mathrm{FeCl}_{2}$ aqueous solution and (b) $\mathrm{Fe} 2+$ is supplied by Fe electrode dissolution. Reprinted with permission from N. Shirai et al., Jpn. J. Appl. Phys., Part 1 56, 076201, 2017. Copyright 2017 Japan Society of Applied Physics. ${ }^{47}$ 
conventional electrolysis conditions. When positive metal ions dissolve into the liquid phase, nano-sized metallic particles form at the surface of the solution as a result of interactions between the ions and plasma-generated electrons; alternatively, they may result from the impact of the plasmagenerated positive ion irradiation.

Magnetic nanoparticles were produced by igniting the atmospheric-pressure plasma at the interface with the $\mathrm{FeCl}_{2}$ aqueous solution; the voltage applied reached $5 \mathrm{kV}$, and the discharge voltage was about $500 \mathrm{~V}$. The plasma was ignited in the direction of the gas flow (in this example, He). In order to control the chemical composition of the plasma-generated species, a shielding gas was flown around the plasma to limit the contamination of the plasma with the air. The particles were synthesized beneath the plasma cathode and collected using a permanent magnet. The magnetic nanoparticles were $200-300 \mathrm{~nm}$ in diameter, composed of magnetite $\mathrm{Fe}_{3} \mathrm{O}_{4}$. It is important to note that the plasma was an imperative prerequisite for the magnetite synthesis, since only $\mathrm{Fe}^{2+}$ could be found in the $\mathrm{FeCl}_{2}$ aqueous solution; then, $\mathrm{Fe}^{2+}$ was oxidized to $\mathrm{Fe}^{3+}$ at the liquid-plasma interface, thus enabling the synthesis of magnetic nanoparticles.

$\checkmark$ Plasma is an imperative prerequisite for the synthesis of nanoscaled magnetite

The liquid-plasma interface is evidently a complex, agile system with many degrees of freedom; naturally, the attempts to add one more degree of freedom to the system were made and were successful. Specifically, complex AuAg core-shell nanoparticles were fabricated by interfacing the atmospheric pressure micro-discharge with flowing liquid precursors.

Figure 29 depicts one such continuous flow setup optimized for the production of $\mathrm{Au}-\mathrm{Ag}$ core-shell particles, which comprises a microscale nozzle, which performs as an anode, and through which the solution, i.e., the flowing liquid cathode is passed at the rate of $3 \mathrm{ml} \cdot \mathrm{min}^{-1}$. Argon is used as a processing gas to generate the plasma jet, at the constant gas flow rate of $120 \mathrm{sccm}$. The cathode is an aqueous solution of $\mathrm{HAuCl}_{4}$ and potentially other additives. The distance of $5 \mathrm{~mm}$ separates the surface of the liquid cathode from the nozzle anode. The potential of $1200 \mathrm{~V}$ was supplied to both electrodes to initiate the discharge, which was then sustained at $45 \mathrm{~mA}$ discharge current. Gelatine was used to stabilize the AuNPs. ${ }^{55}$

\section{$\checkmark$ Adding one more degree of freedom to plasma- liquid interface was successful}

Application of ultrasonic energy to liquids could also be a powerful tool for nanosynthesis. Indeed, efficient nucleation and growth of gold nanowires in the nanoporous membrane realized by a powerful ultrasonic beam was recently demonstrated. ${ }^{66}$ Interestingly, this technology actually combines two distinct processes, namely, the additive (growth) and subtractive (etching) techniques. Fast and uncomplicated, sonochemical synthesis can be performed at ambient temperature in the absence of a catalyst to produce desirable nanomaterials. For instance, ultrasonicating the nanoporous alumina membranes immersed in gold aqueous solution of $\mathrm{HAuCl}_{4}$ by ultrasonic power with the density of $35 \mathrm{~mW} \cdot \mathrm{ml}^{-1}$ and the ultrasonic frequency of $213 \mathrm{kHz}$ produced gold nanowires that were very thin and long, at $\sim 20$ and $30 \mathrm{~nm}$, respectively. The particles were crystalline in nature and were nucleated and grown within the pores of the anodic alumina membrane, suggesting a potential means for control over their size and distribution. On the surface of the membrane, the wires were organized in the form of a forest with high density.

The mechanism of nanowire formation begins with an etching of the pre-existing pores within the alumina membrane to form channels that are open on both sides of the membrane. These channels form a template within which particles nucleate and from which the growing wire is

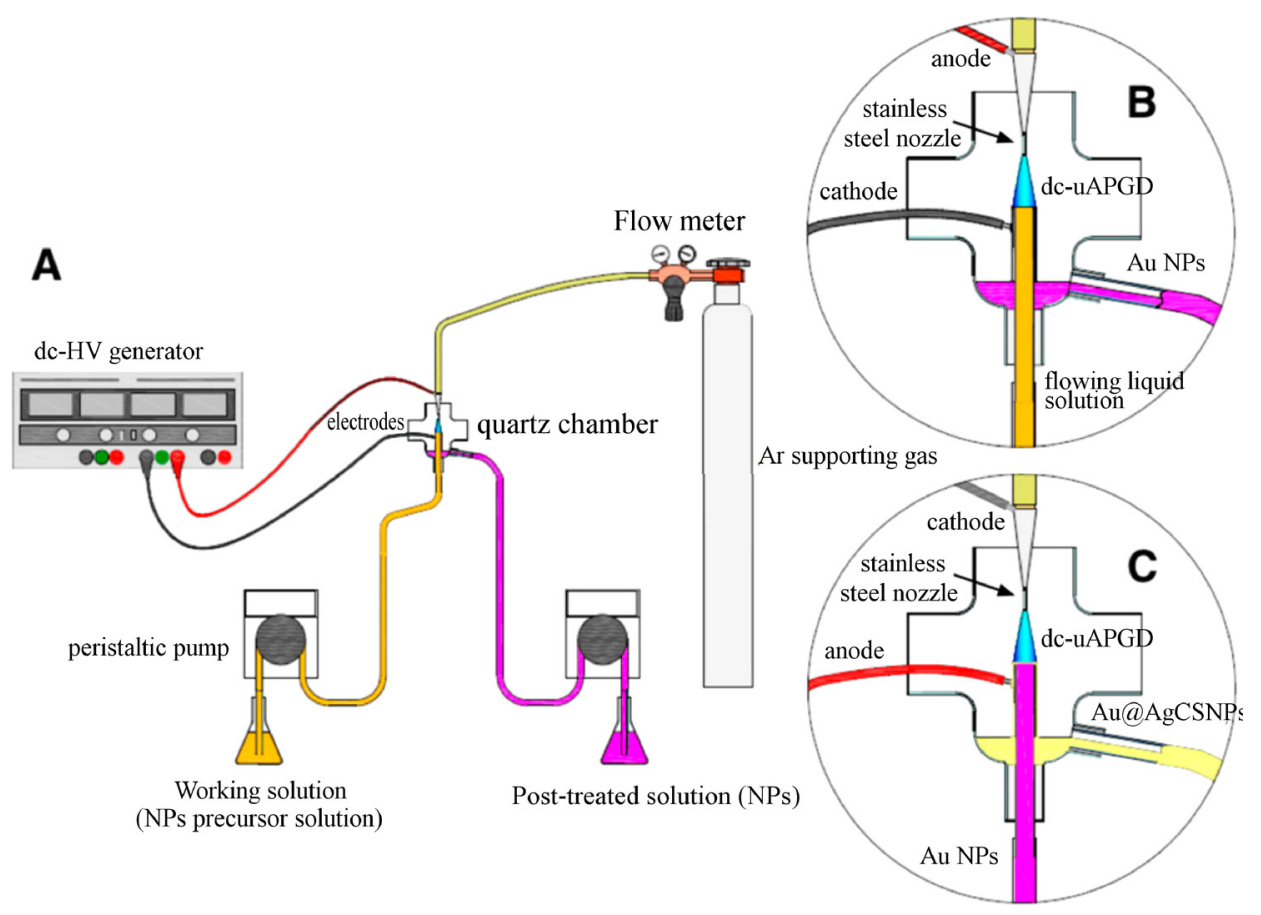

FIG. 29. A schematic representation of the dc- $\mu$ APGD systems used to fabricate the Au-Ag core-shell nanoparticles. (a) An overall setup of the DC atmospheric pressure microdischarge; (b) A close up of the reactor used to generate the microdischarge between a flowing liquid cathode and an Ar nozzle microjet anode for the AuNPs production; (c) A close up of the reactor with the reverse polarity, i.e., using a flowing liquid anode and an Ar nozzle microjet cathode, for the production of $\mathrm{Au}-\mathrm{Ag}$ core-shell nanoparticles. Reprinted with permission from A. Dzimitrowicz et al., Materials 9, 268 (2016). Copyright 2016 Author(s), licensed under the terms and conditions of the Creative Commons Attribution (CC-BY) license. 

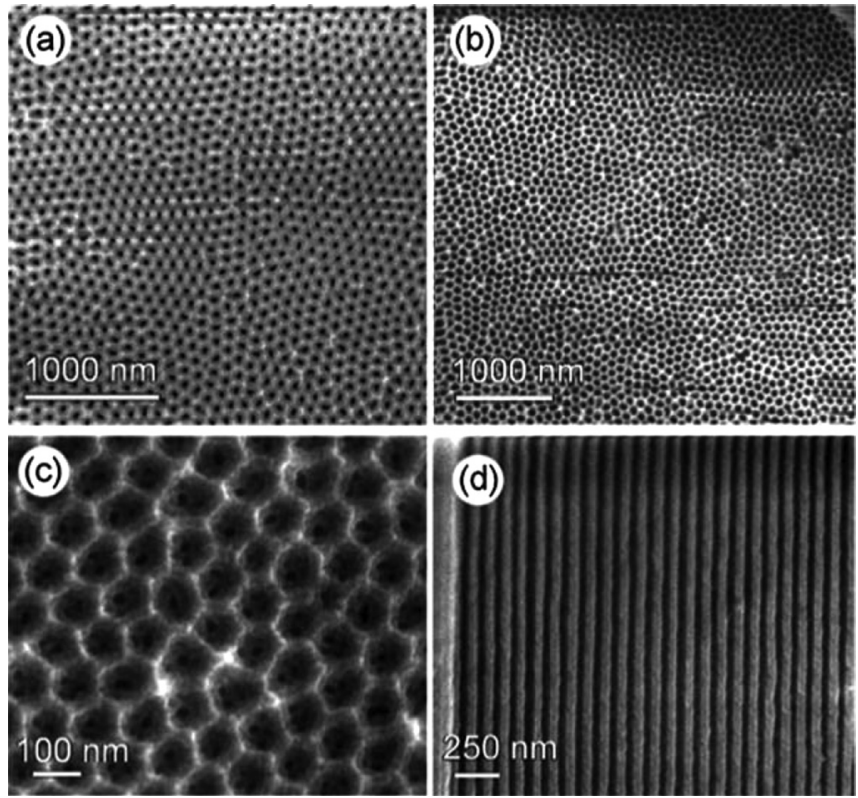

FIG. 30. Characterization of the anodic aluminum oxide membrane. SEM images of the membrane surface after anodization (a) and sonochemical etching [(b) and (c)] show highly oriented hexagonal nanopores covering the entire surface of the membrane. A SEM image of the cross-section of the AAO membrane (d) demonstrates straight, long, perfectly shaped channels. Reprinted with permission from J. Fang et al., J. Mater. Chem. C 1, 1727 (2013). Copyright 2013 RSC.

extruded via acoustic cavitation. The latter denotes the process whereby oscillating cavitational bubbles drive the growing wire out of the pores, thereby affecting their final thickness. This technique can evidently be used for the synthesis of other types of metal nanowires with high degree of control over their structure, dimension, and density. Such materials have a wide range of possible uses, including in nanoscale electronics, fluidics, and optics applications.

$\checkmark$ Powerful ultrasonic energy, causing intense cavitation, also could promote the growth

\section{Subtractive processes at nanoscale: Nanoporous, sharp, and complex structures}

Above outlined additive nanosynthesis is not the only possible application of high density energy to liquids and liquid interfaces. Subtractive manufacturing at nanoscale, in liquids and liquid interfaces, could also be efficient in the fabrication of unique structures. In this case, no material aggregation or nucleation is used, but in contrast, a selective removal of the material under the action of various types of energy in liquids and at the liquid interfaces is utilized. Naturally, the typical examples of this technology will include the fabrication of various porous structures, sharp reaction-active edges with the activated dangling bonds, and similar systems.

We will consider here the two important examples, namely, the fabrication of ordered nanoporous alumina films which could be used as excellent fine filters, nanocapillary systems, and even nanoreactors for synthesis of unique structure without pre-set templates; and sharp porous structures. A typical example of the highly ordered structure, the anodic aluminum oxide membrane is shown in Fig. 30. Interestingly, the two above-mentioned techniques were used to produce the highly ordered nanoporous membranes, namely, the electrolytic anodization of a thin $(100 \mathrm{~mm})$ aluminum foil and ultrasonic etching. The reason for this is the higher degree of controllability which the ultrasonication can provide. The characteristics of the ultrasonic oscillations, specifically their power density and frequency, were optimized to deliver the maximum rate of metal reduction, and concomitantly, to deliver rapid increases in temperature in excess of $1000 \mathrm{~K}$, which spiked as a result of the collapse of cavitation bubbles. ${ }^{57}$ Figure 30(d) clearly shows excellent uniformity and definition of thus-produced straight channels, with the resultant membranes having exceedingly thin (approximately $5 \mathrm{~nm}$ ) walls separating the nanopores.

Sharp structures were produced in the setup type 2, shown in Fig. 27 and described herein. As it was mentioned above, setup type 1 has produced fine, highly crystalline nanoparticles. The setup type 2 differed from type 1 by the plasma-liquid interface; interestingly, this completely changes the type of outcome. Indeed, as shown in Fig. 31, the treatment resulted in uncommon membrane morphology. Electron microscopy and photoelectron spectroscopy studies revealed an extensively etched alumina membrane, with a very high degree of porosity. However, unlike the round regular pores that arise from typical anodization of the alumina
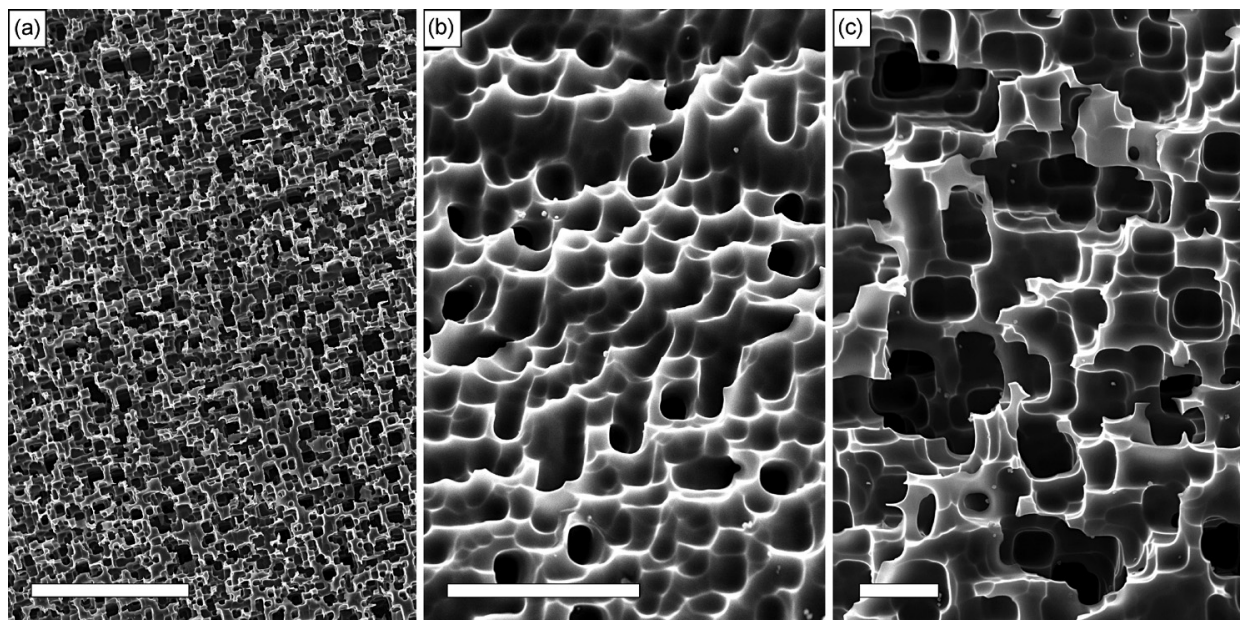

FIG. 31. SEM images of anodized surfaces after the atmospheric plasma jet treatment in oxalic acid in setup 2. A highly porous alumina layer was produced. Scale bars are 10, 2, and $1 \mu \mathrm{m}$, respectively. Reprinted with permission from J. Fang et al., IEEE Trans. Plasma Sci. 43, 765 (2015). Copyright 2015 IEEE. 
foils, ${ }^{66}$ the pores resulting from the direct contact of plasma and the surface of the alumina were irregular in shape with an approximate diameter of $1 \mu \mathrm{m}$ and very sharp edges. Furthermore, they did not conform to the hexagonal pattern typically observed on the alumina templates, nor did they produce straight channels spanning the thickness of the membrane. Indeed, the resulting structure was more similar to that of foam, with a large number of pores separated by alumina walls of very low thickness. Potential applications for such material include those that require very high uptake of the material and/or subsequent protection of the absorbed material from the negative impact of the environment, e.g., protecting of the entrapped biocatalysts from interactions that can reduce their efficacy. ${ }^{34}$

$\checkmark$ Type of plasma-liquid interface completely changes the outcome from additive type (fabrication of nanoparticles) to subtractive type (fabrication of sharp structure)

\section{Bioactivation at plasma-liquid interfaces}

Finally, we will consider an interesting example of the bio-activation of liquid media through exposure to an atmospheric plasma jet; interfacial phenomena also play a key role here. There is a large number of biomolecules that can have attractive biological properties, e.g., broad spectrum antibacterial activity; however, in many instances, it is not sufficient to deliver a sufficiently large reduction in the number of bacteria at the doses that are safe to humans or animals. This limits their applicability in many real-life applications. Plasmagenerated physical, e.g., UV, and chemical effects have the potential to induce desirable alterations in the chemical composition, structure, and such biochemical reactivity of these molecules. ${ }^{185}$ For example, a single brief exposure of aqueous solutions containing a mildly antimicrobial terpinen-4-ol molecule significantly improved the efficacy of the solution against a common pathogenic microorganism (Fig. 32). The attractive features of this approach include relative simplicity of the process and speed with which activation is attained. The properties of the plasma jet and the chemical composition of the solution will directly affect the outcomes. In this example, an atmospheric plasma jet ${ }^{186}$ was produced using Ar and was capable of generating the maximum irradiance of $5 \mathrm{~W} /$ $\mathrm{cm}^{2}$ (within $260-360 \mathrm{~nm}$ range). ${ }^{186}$ The jet was very stable; ${ }^{58}$ it directly contacted the surface of the solution without inducing any significant change in the temperature of the solution upon treatment. $^{187}$

The exact nature of the chemical transformations that can take place upon exposure to plasma-produced mild heating, UV radiation, and reactive chemical species is not fully understood; ${ }^{188,189}$ however, the likely processes include to molecule activation, formation of isomers and oxides, dehydrogenation, synthesis of oligomeric and polymer-like structures, and potentially thermal re-arrangements. ${ }^{190}$ Considering that any number of such reactions can take place, post-treatment, the resulting solution may be enriched with a range of biochemically active derivative molecules, reactive oxygen species, and oxidation ions. In the case of monoterpenes, such as that examined in this example, the chemical reaction pathway may being with $\mathrm{RH} \rightarrow \mathrm{R} \bullet+\mathrm{H} \bullet$ and $\mathrm{ROOH}+\mathrm{RH} \rightarrow \mathrm{R} \bullet+\mathrm{H}_{2} \mathrm{O}+\mathrm{RO} \bullet,{ }^{191}$ with the resulting radicals interacting with plasma-generated ${ }^{3} \mathrm{O}_{2}$ and ${ }^{3} \mathrm{O}_{2}$ species as $\mathrm{R} \bullet+{ }^{3} \mathrm{O}_{2} \rightarrow \mathrm{ROO} \bullet \quad$ and $\mathrm{RO} \bullet+{ }^{3} \mathrm{O}_{2} \rightarrow \mathrm{R}^{\prime}=\mathrm{O}$ $+\mathrm{H}_{2} \mathrm{O} \cdot{ }^{192} \mathrm{Next}$, the highly reactive peroxide can remove weakly bonded $\alpha H(\mathrm{ROO} \bullet+\mathrm{RH} \rightarrow \mathrm{ROOH}+\mathrm{R} \bullet)^{31}$ as well as engage in a wide range of other reactions yielding a greater variety of relatively long-lived chemistries, e.g., $\mathrm{ROO} \bullet+\mathrm{R} \rightarrow \mathrm{ROOR} \bullet, \mathrm{ROOR} \bullet \longrightarrow \mathrm{R}>\mathrm{O}+\mathrm{RO} \bullet, \mathrm{RO} \bullet+\mathrm{RH}$ (a)

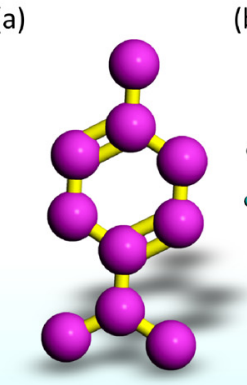

Terpinen-4-ol

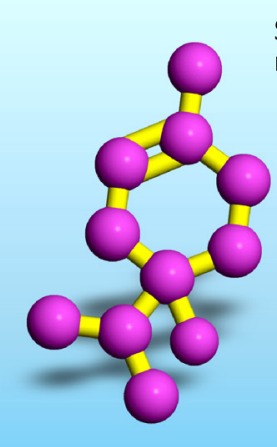

$\mathrm{\text {-terpinene }}$ (b)

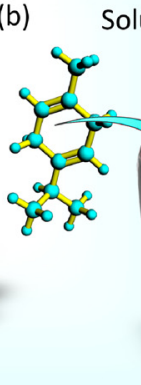

Solutic

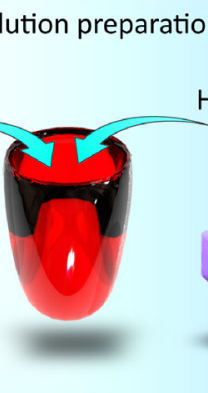

urvival rate estimated by counting CFU number after $18 \mathrm{~h}$ of incubation at $37^{\circ} \mathrm{C}$

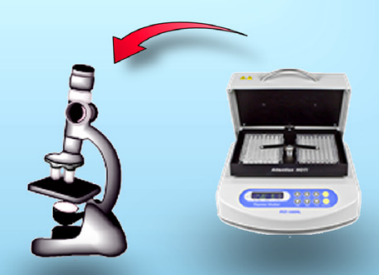

$\mathrm{HO}$

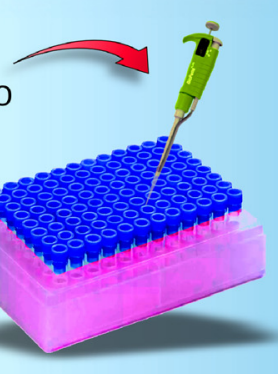

Dispersion to well matrix
Atmospheric pressure plasma activation
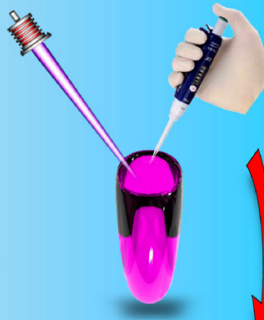

$5 \mathrm{~W} \times \mathrm{cm}^{-2}$

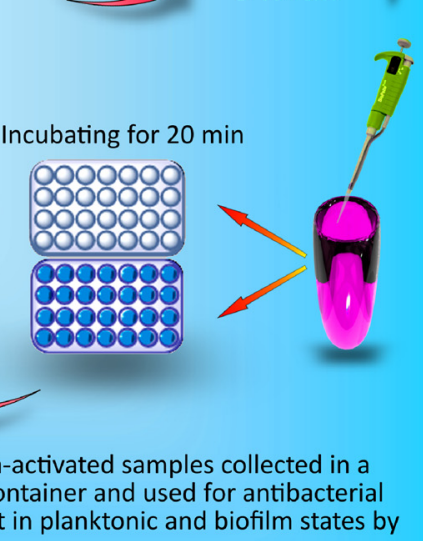

FIG. 32. Two types of secondary metabolites (a) in aqueous solutions are activated using atmosphericpressure plasma jet (b). The jet is made to contact the surface of the solution and maximum exposure of $5 \mathrm{~W} \times \mathrm{cm}^{-2}$ for $5 \mathrm{~min}$ in each well. Thus-activated solutions demonstrated antibacterial activity against pathogenic bacteria in planktonic and biofilm states. Reprinted with permission from K. Bazaka et al., Nano Futures 1, 025002 (2017). Copyright 2017 IOP. 
$\rightarrow \mathrm{ROH}+\mathrm{R} \bullet$, and $\mathrm{ROOH}+\mathrm{ROO} \bullet \rightarrow \mathrm{R}^{\prime}=\mathrm{O}^{\prime}+\mathrm{HO} \bullet+\mathrm{ROOH}$, which can remain or recombine as $\mathrm{R} \bullet+\mathrm{R} \bullet \rightarrow \mathrm{RR}, \mathrm{ROO} \bullet+\mathrm{R} \bullet$ $\rightarrow \mathrm{ROOR}$, and $\mathrm{ROO} \bullet+\mathrm{ROO} \bullet \rightarrow \mathrm{ROH}+\mathrm{R}^{\prime}=\mathrm{O}+{ }^{3} \mathrm{O}_{2}$. Most of the aforementioned chemical products can engage into interactions with the living cell, potentially increasing the desirable antibacterial effect.

\section{OUTLOOK AND PERSPECTIVES}

The strong potential for and bright future of technologies resulting from non-equilibrium, highly energetic processes in liquids and at gas-liquid interfaces are quite apparent nowadays. This paper has outlined numerous examples of unique outcomes produced by energetic processes at interfaces, stressing some features that hardly could be achievable in other environments and processes. Further progress in this technique is expected, with the consolidated effort from physical, chemical, and materials science researchers.

However, researchers and engineers involved into the relevant studies and design projects still face substantial scientific and technological challenges. In general, the whole spectrum of the current problems could be subdivided to the two main groups, namely:

\section{$\checkmark$ Understanding-What happens at interfaces? $\checkmark$ Skills-How to master this promising technique?}

Indeed, plasma and other kinds of energy in liquids form very efficient configurations capable of generating various sophisticated materials and metamaterials of various shapes, chemical composition, structures, and sizes. However, simultaneous presence of the four states of matter makes the understanding of the physics rather challenging. Moreover, transient character and fugacity of many involved processes make their studies an extremely hard task that requires involvement of very complex equipment and procedures. A characteristic example are the attempts to look close into the processes in pulsed-laser synthesis of nanoparticles in liquid; simultaneous application of fast X-ray radiography, scanning small-angle X-ray scattering, and pulsed lasers was utilized to examine the details of these fasts, complicated, intertangled processes (Fig. 33). Major progresses in the understanding of the related phenomena are achieved, but further progress is still needed to ensure better control over this technique.

Discharge ignition in liquids is still pending a clear description and better understanding. The lack of performant diagnostics capable of showing the transition from a liquid phase to a dense ionized gas is a major hindrance to an accurate model. The presence of molecules containing several atoms in liquefied rare gases is likely, owing to a very high pressure that prevails at the first stage of plasma formation. The dynamics of the volume is only roughly estimated, the interface between liquid and gas being too blurry. This medium is also far from equilibrium, optically thick and probably loaded in metallic species from the electrodes from the beginning of the process, which leads to a new difficulty: understand the interaction between the plasma and the electrodes.

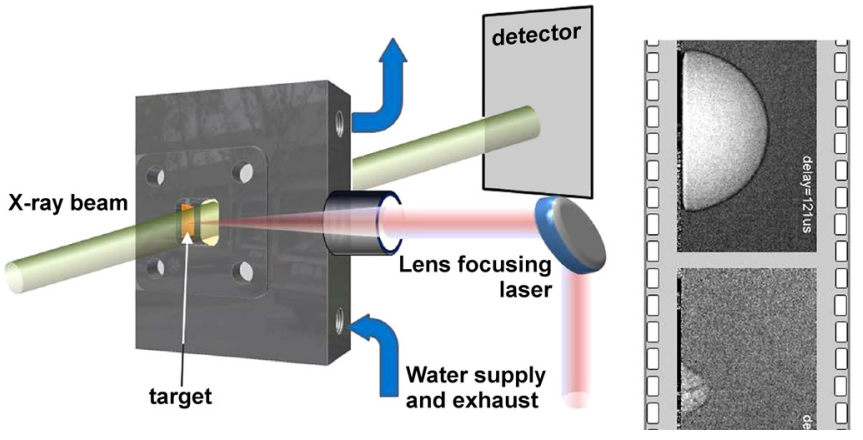

FIG. 33. How to look deeper into ultra-fast processes initiated by dense energy applied to liquids and liquid-gas interfaces? This is a non-trivial problem. Material distribution in the process of the ablation during pulsedlaser assisted nanoparticle generation in solutions is visualized using radiographic and scanning SAXS tracking enabled by a wide white X-ray beam and a monochromatic pencil beam, respectively. Reprinted under Creative Commons Att. 4.0 Int. License from S. Ibrahimkutty et al., Sci. Rep. 5, 16313, 2015. ${ }^{193}$ Copyright 2015, Authors.

Among other fundamental questions, the energy and matter transfer and interchange in the energetic processes in liquids and at the interfaces is still underexplored. Apparently, these processes are key drivers and routes for the nanosynthesis in liquids. However, the details of nanoparticle formation in liquids under action of, e.g., laser or discharge plasma is still not completely understood, and the main processes involved in, e.g., nucleation and growth in bubbles are still questions. Indeed, plasmas in liquids is able to generate a wide range of nanostructured shapes ranged from 1D nanodots to complex metamaterials, see numerous examples in the text of this review and cited herein. Apparently, it is critical to understand the underlying mechanisms to control the shape and composition of nanostructures. ${ }^{194,195}$

Material supply to the nucleation and growth zone is one more key process that is not completely clear, and thus the mechanisms underlying the electrode erosion could be critical to control the shape of growing nanostructures. Indeed, while experiments in liquid nitrogen generate 2D nanoparticles, same materials in water form spherical 1D nanoparticles, thus evidencing the key role of evaporation. Heating metallic electrodes above melting point may take a few hundreds of nanoseconds for most of metals, but metallic vapor appear well before this time lapse; ${ }^{196,197}$ electrode erosion through bursts of tiny protrusions crossed by intense currents may be responsible for the effect.

The evolution of gas bubble in liquid and the related processes are also far from the comprehensive understanding. A gas bubble is usually formed shortly after plasma ignition, and the mechanisms of nanostructure transfer from the gas bubble to liquid phase remains poorly understood, although it is of critical importance for the understanding of the final composition and microstructure of liquid-formed materials. It has been thought for long that nanostructures were transferred into the liquid at the beginning of the first bubble collapse; however, the nanostructures can be confined on the target surface in laser ablation, thus they agglomerate under the action of bubble collapse pressure. ${ }^{193,198}$ This could be of a particular interest for a pin-to-pin configuration 
which implements almost "surface-less" conditions, and the agglomeration might be very different than in the case of laser ablation.

Chemical composition of liquid-synthesized nanostructure is one more issue that demands closer studies. The use of highly energetic processes in liquids is extremely efficient for the generation of metastable materials; nevertheless, alloying just by using electrodes of different materials is not efficient. The use of micro-powder and the synergy of plasmas and laser appear to be very promising for alloying NPs. This approach deserves to be explored deeply for different systems of materials. It could also be of interest to form purely defined compounds far from equilibrium.

\section{$\checkmark$ Synergistic effects of several types of energy could be one of the future ways of developments}

Towards individualized NPs analysis, polydispersity and extremely complex structure at nanoscale (like multilayer and core-shell compositions) of liquid-synthesized nanostructures and materials makes them complicated to characterize in terms of end-user properties. Therefore, it is often crucial to be able to individualize the analysis of nanostructures in terms of their properties. This is already possible for optical and catalytic properties. These diagnostics are of great interest to state on possible application of nanostructures and also to elucidate some peculiarities of nanoscale materials. ${ }^{199-201}$

One more important aspect that warrants discussion is the non-equilibrium state of the media. Indeed, the nonequilibrium state in media excited by plasma or similar powerful effects could be quite short-living, with the nanosecond pulsed discharges in dielectric liquids coming to equilibrium after a couple of ns only (except in the case of hydrogen, where microseconds are needed). Thus, the question of the media being far from equilibrium is not that apparent, especially for the case of high pressure and high temperature media, where conditions are favorable for ultrafast processes relaxing to equilibrium. Hence, the non-equilibrium state should be estimated for every specific medium, environment, and type of excitation power; a model approach established by Griem or that by Fujimoto and McWhirter could be employed for this purpose. ${ }^{202,203}$

To conclude, we try here to formulate several high priority problems that follow from the above examination:

$\checkmark$ Ensuring better understanding of the fundamental processes involved in liquid-energy interactions, material and energy transfer in liquids, as well as nucleation and growth of nanostructures in liquids and at the interfaces;

$\checkmark$ Development of detailed, experimentally proven models capable of predicting the outcomes and routes for complex techniques, that are difficult or impossible to implement under the existing experimental conditions;

$\checkmark$ Developing innovative characterization techniques, capable of analyzing the properties of nano-, meta-, and complex hierarchical materials at various scales.

Further progress in the liquid-based materials treatment techniques could be ensured, taking these milestones into account.

\section{CONCLUDING REMARKS}

In this focused review, we have briefly outlined various aspects of material treatment and activation in liquids and at gas-liquid interfaces. Key physical effects, such as electrohydraulic shocks in liquids, laser-induced shock waves, electrochemical reactions in liquids interfacing the electrodes and glow discharge plasma, processes at liquids interfacing the biological tissues and biofilms, ultrasonic and synergistic effects in energy-exposed liquids, and some others were considered. Examples of practical realization were demonstrated, and the major challenges and problems were indicated.

\section{ACKNOWLEDGMENTS}

This work was supported in part by the following funds and organizations: OSTIn-SRP/EDB, National Research Foundation, and AcRF (Rp6/16 Xs) Singapore; T. B. and A. N. are grateful to "Agence Nationale de la Recherche" for financial support through "CEENEMA" project (ANR-15CE05-0005); I. L. acknowledges the support from the School of Chemistry, Physics and Mechanical Engineering, Science and Engineering Faculty, Queensland University of Technology.

${ }^{1}$ F. Gorrini, M. Cazzanelli, N. Bazzanella, R. Edla, M. Gemmi, V. Cappello, J. David, C. Dorigoni, A. Bifone, and A. Miotello, "On the thermodynamic path enabling a room-temperature, laser-assisted graphite to nanodiamond transformation," Sci. Rep. 6, 35244 (2016).

${ }^{2}$ Y. Yuan, K. Amine, J. Lu, and R. Shahbazian-Yassar, "Understanding materials challenges for rechargeable ion batteries with in situ transmission electron microscopy," Nat. Commun. 8, 15806 (2017).

${ }^{3}$ I. Levchenko, I. I. Beilis, and M. Keidar, "Nanoscaled metamaterial as an advanced heat pump and cooling media," Adv. Mater. Technol. 1, 1600008 (2016).

${ }^{4}$ G. Wehmeyer, T. Yabuki, C. Monachon, J. Wu, and C. Dames, "Thermal diodes, regulators, and switches: Physical mechanisms and potential applications," Appl. Phys. Rev. 4, 041304 (2017).

${ }^{5}$ P. Heremans, A. K. Tripathi, A. de Jamblinne de Meux, E. C. P. Smits, B. Hou, G. Pourtois, and G. H. Gelinck, "Mechanical and electronic properties of thin-film transistors on plastic, and their integration in flexible electronic applications," Adv. Mater. 28, 4266 (2016).

${ }^{6}$ A. D. Stiff-Roberts and W. Ge, "Organic/hybrid thin films deposited by matrix-assisted pulsed laser evaporation (MAPLE)," Appl. Phys. Rev. 4, 041303 (2017).

${ }^{7}$ S. Walia, C. M. Shah, P. Gutruf, H. Nili, D. R. Chowdhury, W. Withayachumnankul, M. Bhaskaran, and S. Sriram, "Flexible metasurfaces and metamaterials: A review of materials and fabrication processes at micro- and nano-scales," Appl. Phys. Rev. 2, 011303 (2015).

${ }^{8}$ I. Levchenko, S. Xu, G. Teel, D. Mariotti, M. L. R. Walker, and M. Keidar, "Smart nanomaterials in space: Recent progress in electric propulsion systems for small satellites," Nat. Commun. 9, 879 (2018).

${ }^{9}$ I. Levchenko, K. Bazaka, Y. Ding, Y. Raitses, S. Mazouffre, T. Henning, P. J. Klar, S. Shinohara, J. Schein, L. Garrigues, M. Kim, D. Lev, F. Taccogna, R. W. Boswell, C. Charles, H. Koizumi, S. Yan, C. Scharlemann, M. Keidar, and S. Xu, "Space micropropulsion systems for Cubesats and small satellites: From proximate targets to furthermost frontiers," Appl. Phys. Rev. 5, 011104 (2018).

${ }^{10} \mathrm{D}$. Terrier, L. Heracleous, and S. Gonzalez, "Enabling paradigm change and agility at NASA's Johnson Space Center-Interview with Chief Technology Officer, Douglas Terrier," Space Policy 39-40, 20-25 (2017).

${ }^{11}$ K. Lemmer, "Propulsion for CubeSats," Acta Astronaut. 134, 231 (2017). ${ }^{12}$ K. Sankaran, L. Cassady, A. D. Kodys, and E. Y. Choueiri, "A survey of propulsion options for cargo and piloted missions to Mars," Ann. NY Acad. Sci. 1017, 450 (2004). 
${ }^{13}$ I. Levchenko, K. Bazaka, M. Keidar, S. Xu, and J. Fang, "Hierarchical multi-component inorganic metamaterials: Intrinsically driven selfassembly at nanoscale," Adv. Mater. 30, 1702226 (2018).

${ }^{14}$ O. Baranov, K. Bazaka, H. Kersten, M. Keidar, U. Cvelbar, S. Xu, and I. Levchenko, "Plasma under control: Advanced solutions and perspectives for plasma flux management in material treatment and nanosynthesis," Appl. Phys. Rev. 4, 041302 (2017).

${ }^{15}$ I. Levchenko, K. K. Ostrikov, J. Zheng, X. Li, M. Keidar, and K. B. K. Teo, "Scalable graphene production: Perspectives and challenges of plasma applications," Nanoscale 8, 10511 (2016).

${ }^{16}$ A. C. Ferrari, F. Bonaccorso, V. Fal'ko, K. S. Novoselov, S. Roche, P. Bøggild, S. Borini et. al., "Science and technology roadmap for graphene, related two-dimensional crystals, and hybrid systems (review)," Nanoscale 7, 4598 (2015).

${ }^{17}$ D. Z. Pai, K. Ostrikov, S. Kumar, D. A. Lacoste, I. Levchenko, and C. O. Laux, "Energy efficiency in nanoscale synthesis using nanosecond plasmas," Sci. Rep. 3, 1221 (2013).

${ }^{18}$ F. Tian, J. Sun, S. L. Hu, and X. W. Du, "Growth dynamics of nanodiamonds synthesized by pulsed-laser ablation,” J. Appl. Phys. 104, 096102 (2008).

${ }^{19}$ V. Švrček, D. Mariotti, and M. Kondo, "Ambient-stable blue luminescent silicon nanocrystals prepared by nanosecond-pulsed laser ablation in water," Opt. Express 17, 520 (2009).

${ }^{20}$ V. Švrček, T. Sasaki, Y. Shimizu, and N. Koshizaki, "Blue luminescent silicon nanocrystals prepared by ns pulsed laser ablation in water," Appl. Phys. Lett. 89, 213113 (2006).

${ }^{21}$ C.-H. Nee, S.-L. Yap, T.-Y. Tou, H.-C. Chang, and S.-S. Yap, "Direct synthesis of nanodiamonds by femtosecond laser irradiation of ethanol," Sci. Rep. 6, 33966 (2016)

${ }^{22}$ H. Kabbara, J. Ghanbaja, C. Noël, and T. Belmonte, "Synthesis of $\mathrm{Cu} @ \mathrm{ZnO}$ core-shell nanoparticles by spark discharges in liquid nitrogen," Nano-Struct. Nano-Objects 10, 22-29 (2017).

${ }^{23}$ J. Patel, L. Nemcova, P. Maguire, W. G. Graham, and D. Mariotti, "Synthesis of surfactant-free electrostatically stabilized gold nanoparticles by plasma-induced liquid chemistry," Nanotechnology 24, 245604 (2013)

${ }^{24}$ I. E. Vlad, O. T. Marisca, A. Vulpoi, S. Simon, N. Leopold, and S. D. Anghel, "Simple approach for gold nanoparticle synthesis using an Arbubbled plasma setup," J. Nanopart. Res. 16, 2633 (2014).

${ }^{25}$ Y. K. Heo, M. A. Bratescu, T. Ueno, and N. Saito, "Synthesis of monodispersed nanofluids using solution plasma," J. Appl. Phys. 116, 024302 (2014)

${ }^{26}$ J. Hieda, N. Saito, and O. Takai, "Exotic shapes of gold nanoparticles synthesized using plasma in aqueous solution," J. Vac. Sci. Technol. A 26, 854 (2008).

${ }^{27} \mathrm{G}$. Saito and T. Akiyama, "Nanomaterial synthesis using plasma generation in liquid," J. Nanomater. 2015, 123696.

${ }^{28}$ K. Bazaka, O. Bazaka, I. Levchenko, S. Xu, E. P. Ivanova, M. Keidar, and K. Ostrikov, "Plasma-potentiated small molecules-Possible alternative to antibiotics?," Nano Futures 1, 025002 (2017).

${ }^{29}$ K. Bazaka, M. V. Jacob, and K. Ostrikov, "Sustainable life cycles of natural-precursor-derived nanocarbons," Chem. Rev. 116, 163 (2016).

${ }^{30}$ Z. Chen, S. Zhang, I. Levchenko, I. I. Beilis, and M. Keidar, "In vitro demonstration of cancer inhibiting properties from stratified selforganized plasma-liquid interface," Sci. Rep. 7, 12163 (2017).

${ }^{31}$ M. Keidar, "Plasma for cancer treatment," Plasma Sources Sci. Technol. 24, 033001 (2015).

${ }^{32}$ F. Müller, S. Grandthyll, S. Gsell, M. Weinl, M. Schreck, and K. Jacobs, "Graphene from Fingerprints: Exhausting the performance of liquid precursor deposition," Langmuir 30, 6114 (2014).

${ }^{33}$ Z. J. Han, A. E. Rider, M. Ishaq, S. Kumar, A. Kondyurin, M. M. M. Bilek, I. Levchenko, and K. Ostrikov, "Carbon nanostructures for hard tissue engineering," RSC Adv. 3, 11058 (2013).

${ }^{34}$ A. Kondyurin, I. Levchenko, Z.-J. Han, S. Yick, A. Mai-Prochnow, J. Fang, K. Ostrikov, and M. M. M. Bilek, "Hybrid graphite film-carbon nanotube platform for enzyme immobilization and protection," Carbon 65, 287 (2013).

${ }^{35} \mathrm{~T}$. Watanabe and T. Tsuru, "Water plasma generation under atmospheric pressure for HFC destruction," Thin Solid Films 516, 4391 (2008).

${ }^{36}$ N. J. Turro, V. Ramamurthy, and J. C. Scaiano, Principles of Molecular Photochemistry: An Introduction (University Science Books, Sausalito, California, USA, 2009), 495 pages. ISBN 978-1-891389-57-3.

${ }^{37}$ A. L. Luna, E. Novoseltceva, E. Louarn, P. Beaunier, E. Kowalska, B. Ohtani, M. A. Valenzuela, H. Remita, and C. Colbeau-Justin, "Synergetic effect of Ni and Au nanoparticles synthesized on titania particles for efficient photocatalytic hydrogen production," Appl. Catal. B 191, 18-28 (2016).

${ }^{38}$ X. Cheng, W. Murphy, N. Recek, D. Yan, U. Cvelbar, A. Vesel, M. Mozetič, J. Canady, M. Keidar, and J. H. Sherman, "Synergistic effect of gold nanoparticles and cold plasma on glioblastoma cancer therapy," J. Phys. D: Appl. Phys. 47, 335402 (2014).

${ }^{39}$ I. Hwang, J. H. Hwang, H. Choi, K.-J. Kim, and D. G. Lee, "Synergistic effects between silver nanoparticles and antibiotics and the mechanisms involved," J. Med. Microbiol. 61, 1719-1726 (2012).

${ }^{40}$ H. Luo, Q. Wang, Y. Deng, T. Yang, H. Ke, H. Yang, H. He, Z. Guo, D. $\mathrm{Yu}, \mathrm{H}$. Wu, and H. Chen, "Mutually synergistic nanoparticles for effective thermo-molecularly targeted therapy," Adv. Funct. Mater. 27, 1702834 (2017).

${ }^{41}$ C. Wang, L. Wu, H. Wang, W. Zuo, Y. Li, and J. Liu, "Fabrication and shell optimization of synergistic $\mathrm{TiO}_{2}-\mathrm{MoO}_{3}$ core-shell nanowire array anode for high energy and power density lithium-ion batteries," Adv. Funct. Mater. 25, 3524-3533 (2015).

${ }^{42}$ S. Wan, F. Xu, L. Jiang, and Q. Cheng, "Superior fatigue resistant bioinspired graphene-based nanocomposite via synergistic interfacial interactions," Adv. Funct. Mater. 27, 1605636 (2017).

${ }^{43}$ T. Belmonte, A. Hamdan, F. Kosior, C. Noël, and G. Henrion, "Interaction of discharges with electrode surfaces in dielectric liquids: Application to nanoparticle synthesis," J. Phys. D: Appl. Phys. 47, 224016 (2014)

${ }^{44}$ Y. Cheng, T. Wang, S. Li, Y. Cheng, J. Cao, and H. Xie, "The effects of anion deposition and negative pulse on the behaviours of plasma electrolytic oxidation (PEO) - A systematic study of the PEO of a Zirlo alloy in aluminate electrolytes," Electrochim. Acta 225, 47 (2017).

${ }^{45}$ T. A. Kareem and A. A. Kaliani, "Glow discharge plasma electrolysis for nanoparticles synthesis," Ionics 18, 315 (2012).

${ }^{46}$ F. Tochikubo, N. Shirai, and S. Uchida, "Liquid-phase reactions induced by atmospheric pressure glow discharge with liquid electrode," J. Phys.: Conf. Ser. 565, 012010 (2014).

${ }^{47}$ N. Shirai, T. Yoshida, S. Uchida, and F. Tochikubo, "Synthesis of magnetic nanoparticles by atmospheric-pressure glow discharge plasmaassisted electrolysis," Jpn. J. Appl. Phys., Part 1 56, 076201 (2017).

${ }^{48}$ P. Giridhar, S. Z. E. Abedin, and F. Endres, "Electrodeposition of nanocrystalline aluminium, copper, and copper-aluminium alloys from 1butyl-1-methylpyrrolidinium trifluoromethyl-sulfonate ionic liquid," J. Solid State Electrochem. 16, 3487 (2012).

${ }^{49}$ D. Mariotti, J. Patel, V. Svrcek, and P. Maguire, "Plasma-liquid interactions at atmospheric pressure for nanomaterials synthesis and surface engineering," Plasma Process. Polym. 9, 1074 (2012).

${ }^{50}$ P. J. Bruggeman, M. J. Kushner, B. R. Locke, J. G. E. Gardeniers, W. G. Graham, D. B. Graves, R. C. H. M. Hofman-Caris et al., "Plasma-liquid interactions: A review and roadmap," Plasma Sources Sci. Technol. 25, 053002 (2016).

${ }^{51}$ L. Lin and Q. Wang, "Microplasma: A new generation of technology for functional nanomaterial synthesis," Plasma Chem. Plasma Process. 35, 925 (2015).

${ }^{52}$ D. Mariotti and R. M. Sankaran, "Microplasmas for nanomaterials synthesis," J. Phys. D: Appl. Phys. 43, 323001 (2010).

${ }^{53}$ T. Belmonte, G. Henrion, and T. Gries, "Nonequilibrium atmospheric plasma deposition,” J. Therm. Spray Technol. 20, 744 (2011).

${ }^{54}$ I. G. Koo, M. S. Lee, J. H. Shim, J. H. Ahn, and W. M. Lee, "Platinum nanoparticles prepared by a plasma-chemical reduction method," J. Mater. Chem. 15, 4125 (2005).

${ }^{55}$ A. Dzimitrowicz, P. Jamroz, M. Nyk, and P. Pohl, "Application of direct current atmospheric pressure glow microdischarge generated in contact with a flowing liquid solution for synthesis of Au-Ag core-shell nanoparticles," Materials 9, 268 (2016).

${ }^{56}$ J. Fang, I. Levchenko, and K. Ostrikov, "Atmospheric plasma jetenhanced anodization and nanoparticle synthesis," IEEE Trans. Plasma Sci. 43, 765 (2015).

${ }^{57}$ K. Okitsu, M. Ashokkumar, and F. Grieser, "Sonochemical synthesis of gold nanoparticles: Effects of ultrasound frequency," J. Phys. Chem. B 109, 20673 (2005).

${ }^{58}$ J. Fang, I. Levchenko, A. Mai-Prochnow, M. Keidar, U. Cvelbar, G. Filipic, Z. J. Han, and K. Ostrikov, "Protein retention on plasmatreated hierarchical nanoscale gold-silver platform," Sci. Rep. 5, 13379 (2015).

${ }^{59}$ R. Zhou, R. Zhou, J. Zhuang, Z. Zong, X. Zhang, D. Liu et al., "Interaction of atmospheric-pressure air microplasmas with amino acids 
as fundamental processes in aqueous solution," PLoS one 11, e0155584 (2016).

${ }^{60}$ R. G. Nikov, N. N. Nedyalkov, P. A. Atanasov, and D. B. Karashanova, "Laser-assisted fabrication and size distribution modification of colloidal gold nanostructures by nanosecond laser ablation in different liquids," Appl. Phys. A 123, 490 (2017).

${ }^{61}$ N. Lasemi, U. Pacher, C. Rentenberger, O. Bomatí-Miguel, and W. Kau, "Laser-assisted synthesis of colloidal $\mathrm{Ni} / \mathrm{NiO}_{\mathrm{x}}$ core/shell nanoparticles in water and alcoholic solvents," ChemPhysChem 18, 1118-1124 (2017).

${ }^{62}$ Z. K. Liu, Z. Y. Cao, B. W. Deng, Y. F. Wang, J. Y. Shao, P. Kumar, C. R. Liu, B. Q. Wei, and G. J. Cheng, "Ultrafast and scalable laser liquid synthesis of tin oxide nanotubes and its application in lithium ion batteries," Nanoscale 6, 5853-5858 (2014).

${ }^{63}$ X. X. Xu, G. T. Duan, Y. Li, H. W. Zhang, G. Q. Liu, and W. P. Cai, "Synthesis of nano-cubic $\mathrm{ZnSn}(\mathrm{OH})(3)$ based on stannate reaction with liquid laser ablation-induced $\mathrm{ZnO}$ below room temperature," Crystengcomm 15, 6159 (2013).

${ }^{64}$ A. De Giacomo, A. De Bonis, M. Dell'Aglio, O. De Pascale, R. Gaudiuso, S. Orlando, A. Santagata, G. S. Senesi, F. Taccogna, and R. Teghil, "Laser ablation of graphite in water in a range of pressure from 1 to 146 atm using single and double pulse techniques for the production of carbon nanostructures," J. Phys. Chem. C 115, 5123-5130 (2011).

${ }^{65}$ S. S. Nkosi, B. W. Mwakikunga, E. Sideras-Haddad, and A. Forbes, "Synthesis and characterization of potential iron-platinum drugs and supplements by laser liquid photolysis," Nanotechnol. Sci. Appl. 5, 27-36 (2012)

${ }^{66}$ J. Fang, I. Levchenko, K. Ostrikov, and S. Prawer, "Sonochemical nanoplungers: Crystalline gold nanowires by cavitational extrusion through nanoporous alumina," J. Mater. Chem. C 1, 1727 (2013).

${ }^{67}$ C. Kunzmann, G. Schmidt-Bilkenroth, J. Moosburger-Will, and S. Horn, "Microscopic investigation of polyacrylonitrile fiber fibrils separated by ultrasonic etching," J. Mater. Sci. 53, 4693-4704 (2018).

${ }^{68}$ G. A. Husseini, W. G. Pitt, and A. M. Martins, "Ultrasonically triggered drug delivery: Breaking the barrier," Colloids Surf. B 123, 364-386 (2014).

${ }^{69}$ M. Javadi, W. G. Pitt, C. M. Tracy, J. R. Barrow, B. M. Willardson, J. M. Hartley, and N. H. Tsosie, "Ultrasonic gene and drug delivery using eLiposomes," J. Control. Release 167, 92-100 (2013).

${ }^{70}$ S. J. Grainger, J. V. Serna, S. Sunny, Y. Zhou, C. X. Deng, and M. E. ElSayed, "Pulsed ultrasound enhances nanoparticle penetration into breast cancer spheroids," Mol. Pharmacol. 7, 2006-2019 (2010).

${ }^{71}$ J. Fang, I. Levchenko, and K. Ostrikov, "Free-standing alumina nanobottles and nanotubes pre-integrated into nanoporous alumina membranes," Sci. Technol. Adv. Mater. 15, 045004 (2014).

${ }^{72}$ E. Era, H. Çelikkan, and N. Erk, "A novel electrochemical nano-platform based on graphene/platinum nanoparticles/nafion composites for the electrochemical sensing of metoprolol," Sens. Actuators B 238, 779-787 (2017).

${ }^{73}$ H. D. Xiao, J. S. Cui, D. H. Cao, Q. X. Gao, J. Q. Liu, and J. Ma, "Selfstanding nanoporous GaN membranes fabricated by UV-assisted electrochemical anodization," Mater. Lett. 145, 304-307 (2015).

${ }^{74} \mathrm{P}$. Stroeve and N. Ileri, "Biotechnical and other applications of nanoporous membranes," Trends Biotechnol. 29, 259-266 (2011).

${ }^{75}$ N. Burham, A. A. Hamzah, J. Yunas, and B. Y. Majlis, "Electrochemically etched nanoporous silicon membrane for separation of biological molecules in mixture," J. Micromech. Microeng. 27, 075021 (2017).

${ }^{76}$ G. Jeon, S. Y. Yang, and J. K. Kim, "Functional nanoporous membranes for drug delivery," J. Mater. Chem. 22, 14814-14834 (2012).

${ }^{77}$ M. Bresin, A. Botman, S. J. Randolph, M. Straw, and J. T. Hastings, "Liquid phase electron-beam-induced deposition on bulk substrates using environmental scanning electron microscopy," Microsc. Microanal. 20, 376-384 (2014).

${ }^{78}$ M. Bresin, A. Chamberlain, E. U. Donev, C. B. Samantaray, G. S. Schardien, and J. T. Hastings, "Electron-beam induced deposition of bimetallic nanostructures from bulk liquids," Angew. Chem. Int. Ed. 52, 8004-8007 (2013).

${ }^{79}$ E. U. Donev and J. T. Hastings, "Electron-beam-induced deposition of platinum from a liquid precursor," Nano Lett. 9, 2715-2718 (2009).

${ }^{80}$ E. U. Donev and J. T. Hastings, "Liquid-precursor electron beam-induced deposition of Pt nanostructures: Dose, proximity, resolution," Nanotechnology 20, 505302 (2009).

${ }^{81}$ L. E. Ocola, A. Joshi-Imre, C. Kessel, B. Chen, J. Park, D. Gosztola, and R. Divan, "Growth characterization of electron-beam-induced silver deposition from liquid precursor," J. Vac. Sci. Technol. B 30, 06 FF08 (2012).

${ }^{82}$ G. Schardein, E. U. Donev, and J. T. Hastings, "Electron-beam induced deposition of gold from aqueous solutions," Nanotechnology 22, 015301 (2011).

${ }^{83}$ T. Taaber, M. Antsov, S. Vlassov, U. Mäeorg, L. Dorogin, M. Järvekülg, K. Saal, and R. Lõhmus, "Formation and characterization of microcantilevers produced from ionic liquid by electron beam irradiation," J. Mol. Liquids 229, 45-50 (2017).

${ }^{84}$ J. M. Grogan, N. M. Schneider, F. M. Ross, and H. H. Bau, "Bubble and pattern formation in liquid induced by an electron beam," Nano Lett. 14, 359--364 (2014).

${ }^{85}$ L. Boehme, M. Bresin, A. Botman, J. Ranney, and J. T. Hastings, "Focused electron beam induced etching of copper in sulfuric acid solutions," Nanotechnology 26, 495301 (2015).

${ }^{86}$ U. Y. Lau, S. S. Saxer, J. Lee, E. Bat, and H. D. Maynard, "Direct write protein patterns for multiplexed cytokine detection from live cells using electron beam lithography," ACS Nano 10, 723-729 (2016).

${ }^{87}$ A. Stoica-Guzun, M. Stroescu, F. Tache, T. Zaharescu, and E. Grosu, "Effect of electron beam irradiation on bacterial cellulose membranes used as transdermal drug delivery systems," Nucl. Instrum. Methods B 265, 434-438 (2007).

${ }^{88} \mathrm{M}$. Vasiliev and T. Vasilieva, "Electron-beam plasma in the production of bioactive agents and drugs," J. Phys. Conf. Ser. 44, 140-145 (2006).

${ }^{89}$ P. Liu, C. Wang, X. Chen, and G. Yang, "Controllable fabrication and cathodoluminescence performance of high-index facets $\mathrm{GeO}_{2}$ micro- and nanocubes and spindles upon electrical-field-assisted laser ablation in liquid," J. Phys. Chem. C 112, 13450 (2008).

${ }^{90}$ E. Giorgetti, P. Marsili, P. Canton, M. Muniz-Miranda, S. Caporali, and F. Giammanco, "Cu/Ag-based bifunctional nanoparticles obtained by one-pot laser-assisted galvanic replacement," J. Nanopart. Res. 15, 1360 (2013).

${ }^{91}$ Z. Zhang, M. Cai, Q. Feng, and Y. Zeng, "Comparison of different laserassisted electrochemical methods based on surface morphology characteristics," Int. J. Adv. Manuf. Technol. 71, 565-571 (2014).

${ }^{92}$ B. W. Mwakikunga, A. Forbes, E. Sideras-Haddad, M. Scriba, and E. Manikandan, "Self assembly and properties of $\mathrm{C}: \mathrm{WO}_{3}$ nano-platelets and $\mathrm{C}: \mathrm{VO}_{2} / \mathrm{V}_{2} \mathrm{O}_{5}$ triangular capsules produced by laser solution photolysis," Nanoscale Res. Lett. 5, 389 (2010).

${ }^{93} \mathrm{P}$. Wagener, J. Jakobi, and S. Barcikowski, "Organic nanoparticles generated by combination of laser fragmentation and ultrasonication in liquid," J. Laser Micro Nanoeng. 6, 59-63 (2011).

${ }^{94}$ L. Escobar-Alarcon, E. Velarde Granados, D. A. Solis-Casados, O. OleaMejia, M. Espinosa-Pesqueira, and E. Haro-Poniatowski, "Preparation of bismuth-based nanosheets by ultrasound-assisted liquid laser ablation," Appl. Phys. A 122, 433 (2016).

${ }^{95}$ L. Escobar-Alarcón, M. E. Espinosa-Pesqueira, D. A. Solis-Casados, J. Gonzalo, J. Solis, M. Martinez-Orts, and E. Haro-Poniatowski, "Twodimensional carbon nanostructures obtained by laser ablation in liquid: Effect of an ultrasonic field," Appl. Phys. A 124, 141 (2018).

${ }^{96}$ A. Pearson, H. Zheng, K. Kalantar-Zadeh, S. K. Bhargava, and V. Bansal, "Decoration of $\mathrm{TiO}_{2}$ nanotubes with metal nanoparticles using polyoxometalate as a UV-switchable reducing agent for enhanced visible and solar light photocatalysis," Langmuir 28, 14470-14475 (2012).

${ }^{97}$ L. Sun, J. Li, C. Wang, S. Li, Y. Lai, H. Chen et al., "Ultrasound aided photochemical synthesis of $\mathrm{Ag}$ loaded $\mathrm{TiO}_{2}$ nanotube arrays to enhance photocatalytic activity," J. Hazard. Mater. 171, 1045-1050 (2009).

${ }^{98}$ Q. Wu, J. Ouyang, K. Xie, L. Sun, M. Wang, and C. Lin, "Ultrasoundassisted synthesis and visible-light-driven photocatalytic activity of $\mathrm{Fe}$ incorporated $\mathrm{TiO}_{2}$ nanotube array photocatalysts," J. Hazard. Mater. 199-200, 410-417 (2012).

${ }^{99}$ M. Nischk, P. Mazierski, Z. Wei, K. Siuzdak, N. A. Kouamee, E. Kowalskac, H. Remita, and A. Zaleska-Medynska, "Enhanced photocatalytic, electrochemical and photoelectrochemical properties of $\mathrm{TiO}_{2}$ nanotubes arrays modified with $\mathrm{Cu}, \mathrm{AgCu}$ and $\mathrm{Bi}$ nanoparticles obtained via radiolytic reduction," Appl. Surf. Sci. 387, 89-102 (2016).

${ }^{100}$ O. Tahiri Alaoui, A. Herissan, C. Le Quoc, M. E. M. Zekri, S. Sorgues, H. Remita et al., "Elaboration, charge-carrier lifetimes and activity of Pd$\mathrm{TiO}_{2}$ photocatalysts obtained by gamma radiolysis," J. Photochem. Photobiol. A 242, 34-43 (2012).

${ }^{101}$ Z. Hai, N. El Kolli, J. Chen, and H. Remita, "Radiolytic synthesis of $\mathrm{Au}-\mathrm{Cu}$ bimetallic nanoparticles supported on $\mathrm{TiO}_{2}$ : Application in photocatalysis," New J. Chem. 38, 5279-5286 (2014). 
${ }^{102}$ A. Kruusing, "Physics and chemistry of laser-liquid-solid interactions," in Handbook of Liquids-Assisted Laser Processing (Elsevier, 2008), Chap. 7, pp. 281-314.

${ }^{103}$ A. Kruusing, "Physics and chemistry of laser-liquid-solid interactions," in Handbook of Liquids-Assisted Laser Processing (Elsevier, 2008), Chap. 3, pp. 69-142.

${ }^{104}$ C. Radu, S. Simion, M. Zamfirescu, M. Ulmeanu, M. Enculescu, and M. Radoiu, "Silicon structuring by etching with liquid chlorine and fluorine precursors using femtosecond laser pulses," J. Appl. Phys. 110, 034901 (2011).

${ }^{105}$ D. Poondi, T. Dobbins, and J. Singh, "A novel laser-liquid-solid interaction technique for synthesis of silver, nickel and immiscible silver-nickel alloys from liquid precursors," J. Mater. Sci. 35, 6237-6243 (2000).

${ }^{106}$ J. Singh, D. Poondi, T. Dobbins, and M. M. Pietro, "Novel nanomaterials synthesis by laser-liquid-solid interaction," Mater. Manuf. Process 23, 228-240 (2008).

${ }^{107}$ N. V. Tarasenko and A. V. Butsen, "Laser synthesis and modification of composite nanoparticles in liquids," Quantum Electron. 40, 986-1003 (2010)

${ }^{108}$ J. Zhou, C. Booker, R. Li, X. Zhou, T.-K. Sham, X. Sun, and Z. Ding, "An electrochemical avenue to blue luminescent nanocrystals from multiwalled carbon nanotubes (MWCNTs),” J. Am. Chem. Soc. 129, 744 (2007).

${ }^{109}$ J. Martin, A. Nominé, F. Brochard, J.-L. Briancon, C. Noël, T. Belmonte, T. Czerwiec, and G. Henrion, "Delay in micro-discharges appearance during PEO of $\mathrm{Al}$ : Evidence of a mechanism of charge accumulation at the electrolyte/oxide interface," Appl. Surf. Sci. 410, 29 (2017).

${ }^{110}$ A. Nominé, S. C. Troughton, A. V. Nominé, G. Henrion, and T. W. Clyne, "High speed video evidence for localised discharge cascades during plasma electrolytic oxidation," Surf. Coat. Technol. 269, 125 (2015).

${ }^{111}$ A. Nominé, A. V. Nominé, N. St, J. Braithwaite, T. Belmonte, and G. Henrion, "High-Frequency-induced cathodic breakdown during plasma electrolytic oxidation," Phys. Rev. Appl. 8, 031001 (2017).

${ }^{112}$ C. S. Dunleavy, J. A. Curran, and T. W. Clyne, "Time dependent statistics of plasma discharge parameters during bulk AC plasma electrolytic oxidation of aluminium," Appl. Surf. Sci. 268, 397 (2013).

${ }^{113}$ S. C. Troughton, A. Nominé, J. Dean, and T. W. Clyne, "Effect of individual discharge cascades on the microstructure of plasma electrolytic oxidation coatings," Appl. Surf. Sci. 389, 260 (2016).

${ }^{114}$ S. Ghosh, B. Bishop, I. Morrison, and R. Akolkar, "Generation of a direct-current, atmospheric-pressure microplasma at the surface of a liquid water microjet for continuous plasma-liquid processing," J. Vac. Sci. Technol. A 33, 021312 (2015).

${ }^{115}$ N. Shirai, S. Uchida, F. Tochikubo, and S. Ishii, "Self-organized anode pattern on surface of liquid or metal anode in atmospheric DC glow discharges," IEEE Trans. Plasma Sci. 39, 2652 (2011).

${ }^{116}$ M. Keidar, D. Yan, I. I. Beilis, B. Trink, and J. H. Sherman, "Plasmas for treating cancer: Opportunities for adaptive and self-adaptive approaches," Trends Biotechnol. (in press).

${ }^{117}$ W. R. Ashby, "Principles of the self-organizing dynamic system," J. Gen. Psychol. 37, 125 (1947).

${ }^{118}$ T. Takeda and J.-S. Chang, "Morphology of high-frequency electrohydraulic discharge for liquid-solution plasmas," IEEE Trans. Plasma Sci. 36, 1158 (2008)

${ }^{119}$ Advanced Plasma Technology, 1st ed., edited by R. d'Agostino, P. Favia, Y. Kawai, H. Ikegami, N. Sato, and F. Arefi-Khonsari (Wiley-VCH Verlag and Co., Weinheim, 2008).

${ }^{120}$ I. V. Timoshkin, R. A. Fouracre, M. J. Given, and S. J. MacGregor, "Hydrodynamic modelling of transient cavities in fluids generated by high voltage spark discharges," J. Phys. D: Appl. Phys. 39, 4808 (2006).

${ }^{121}$ D. Oshita, S. H. R. Hosseini, K. Mawatari, S. M. Nejad, and H. Akiyama, "Two successive shock waves generated by underwater pulse electric discharge for medical applications," IEEE Trans. Plasma Sci. 42, 3209-3214 (2014).

${ }^{122}$ Y. Liu, Z.-Y. Li, X.-D. Li, S.-W. Liu, G.-Y. Zhou, and F.-C. Lin, "Intensity improvement of shock waves induced by liquid electrical discharges," Phys. Plasmas 24, 043510 (2017).

${ }^{123}$ I. I. Kochetkov and A. V. Pinaev, "Shock and detonation waves generated by a wire explosion in liquids and bubbled media," Combust. Explos. Shock Waves 48, 236-244 (2012).

${ }^{124}$ S. Krishnan, A. S. M. A. Haseeb, and M. R. Johan, "Synthesis and growth kinetics of spindly $\mathrm{CuO}$ nanocrystals via pulsed wire explosion in liquid medium," J. Nanopart. Res. 15, 1410 (2013).

${ }^{125}$ P. Kennedy, D. Hammer, and B. Rockwell, "Laser-induced breakdown in aqueous media,” Prog. Quant. Electron. 21, 155-248 (1997).
${ }^{126}$ V. Lazic and S. Jovićević, "Laser induced breakdown spectroscopy inside liquids: Processes and analytical aspects," Spectrochim. Acta B 101, 288-311 (2014).

${ }^{127}$ D. Jang, J.-G. Park, and D. Kim, "Enhancement of airborne shock wave by laser-induced breakdown of liquid column in laser shock cleaning," J. Appl. Phys. 109, 073101 (2011).

${ }^{128}$ C. H. Cho, S. H. Park, Y. W. Choi, and B. G. Kim, "Production of nanopowders by wire explosion in liquid media," Surf. Coat. Technol. 201, 4847-4849 (2007).

${ }^{129} \mathrm{~L}$. Rayleigh, "On the pressure developed in a liquid during the collapse of a spherical cavity," Philos. Mag. 34, 94 (1917).

${ }^{130}$ W. Guo, X. Zheng, G. Yu, J. Zhao, Y. Zeng, and C. Liu, "Investigation of laser induced breakdown in liquid nitromethane using nanosecond shadowgraphy," J. Appl. Phys. 120, 123301 (2016).

${ }^{131}$ S. Sirisky, Y. Yang, W. Wei, and H. J. Maris, "Laser-induced breakdown in liquid helium," J. Low Temp. Phys. 189, 53 (2017).

${ }^{132}$ T. Seto, A. Inoue, H. Higashi, Y. Otani, M. Kohno, and M. Hirasawa, "Phase transition and restructuring of carbon nanoparticles induced by aerosol laser irradiation," Carbon 70, 224-232 (2014).

${ }^{133}$ T. Sun, Y. S. Zhang, B. Pang, D. C. Hyun, M. Yang, and Y. Xia, "Engineered nanoparticles for drug delivery in cancer therapy," Angew. Chem. Int. Ed. 53, 12320-12364 (2014).

${ }^{134}$ R. B. S. Panchangam and T. Dutta, "Engineered nanoparticles for the delivery of anticancer therapeutics," J. Pharm. Drug Deliv. Res. 4, 1 (2015).

${ }^{135}$ T. Ukai, H. Zare-Behtash, and K. Kontis, "Suspended liquid particle disturbance on laser-induced blast wave and low density distribution," Phys. Fluids 29, 126104 (2017).

${ }^{136}$ R. Akolkar and R. M. Sankaran, "Charge transfer processes at the interface between plasmas and liquids," J. Vac. Sci. Technol. A 31, 050811 (2013).

${ }^{137}$ A. Hickling and M. D. Ingram, Trans. Faraday Soc. 60, 783 (1964).

${ }^{138}$ X. Jin, X. Wang, H. Zhang, Q. Xia, D. Wei, and J. Yue, Plasma Chem. Plasma Process. 30, 429 (2010).

${ }^{139}$ B. G. Janesko, G. Scalmani, and M. J. Frisch, "Quantifying solvated electrons' delocalization,” Phys. Chem. Chem. Phys. 17, 18305-18317 (2015).

${ }^{140}$ J. Goodman, A. Hickling, and B. Schofield, "The yield of hydrated electrons in glow-discharge electrolysis," J. Electroanal. Chem. Interfacial Electrochem. 48, 319-322 (1973).

${ }^{141}$ R. Gopalakrishnan, E. Kawamura, A. J. Lichtenberg, M. A. Lieberman, and D. B. Graves, "Solvated electrons at the atmospheric pressure plasma-water anodic interface,” J. Phys. D: Appl. Phys. 49, 295205 (2016).

${ }^{142}$ C. Chen, D. X. Liu, Z. C. Liu, A. J. Yang, H. L. Chen, G. Shama, and M. G. Kong, "A model of Plasma-biofilm and plasma-tissue interactions at ambient pressure," Plasma Chem. Plasma Process. 34, 403 (2014).

${ }^{143}$ P. Rumbach, D. M. Bartels, R. M. Sankaran, and D. B. Go, "The solvation of electrons by an atmospheric-pressure plasma," Nat. Commun. 6, 7248 (2015).

${ }^{144}$ X. T. Deng, J. J. Shi, H. L. Chen, and M. G. Kong, "Protein destruction by atmospheric pressure glow discharges," Appl. Phys. Lett. 90, 013903 (2007).

${ }^{145}$ X. T. Deng, J. J. Shi, and M. G. Kong, "Protein destruction by a helium atmospheric pressure glow discharge: Capability and mechanisms," J. Appl. Phys. 101, 074701 (2007).

${ }^{146}$ W. Van Gaens and A. Bogaerts, J. Phys. D: Appl. Phys. 46, 275201 (2013).

${ }^{147}$ P. R. Chalise, S. Perni, G. Shama, B. M. Novac, I. R. Smith, and M. G. Kong, Appl. Phys. Lett. 89, 153902 (2006).

${ }^{148}$ I. Levchenko, K. Ostrikov, A. E. Rider, E. Tam, S. V. Vladimirov, and S. $\mathrm{Xu}$, "Growth kinetics of carbon nanowall-like structures in lowtemperature plasmas," Phys. Plasmas 14, 063502 (2007).

${ }^{149}$ A. E. Rider, I. Levchenko, and K. Ostrikov, "Surface fluxes of Si and C adatoms at initial growth stages of SiC quantum dots," J. Appl. Phys. 101, 044306 (2007).

${ }^{150}$ A. H. G. Cents, D. W. F. Brilman, and G. F. Versteeg, " $\mathrm{CO}_{2}$ absorption in carbonate/bicarbonate solutions: The Danckwerts-criterion revisited," Chem. Eng. Sci. 60, 5830 (2005).

${ }^{151}$ H. P. Langtangen and A. Logg, "Fundamentals: Solving the Poisson equation," in Solving PDEs in Python, Simula Springer Briefs on Computing, Vol. 3 (Springer, Cham, 2016). DOI: https://doi.org/10.1007/ 978-3-319-52462-7_2

${ }^{152}$ S. Merouani, O. Hamdaoui, Y. Rezgui, and M. Guemini, "Theoretical estimation of the temperature and pressure within collapsing acoustical bubbles," Ultrason. Sonochem. 21, 53-59 (2014). 
${ }^{153}$ S. Merouani, O. Hamdaoui, Y. Rezgui, and M. Guemini, "Computer simulation of chemical reactions occurring in collapsing acoustical bubble: Dependence of free radicals production on operational conditions," Res. Chem. Intermed. 41, 881 (2015).

${ }^{154}$ P. Kanthale, M. Ashokkumar, and F. Grieser, "Sonoluminescence, sonochemistry $\left(\mathrm{H}_{2} \mathrm{O}_{2}\right.$ yield) and bubble dynamics: Frequency and power effects," Ultrason. Sonochem. 15, 143 (2008).

${ }^{155}$ A. L. Zanin, A. W. Liehr, A. S. Moskalenko, and H.-G. Purwins, "Voronoi diagrams in barrier gas discharge," Appl. Phys. Lett. 81, 3338 (2002)

${ }^{156}$ I. Levchenko and K. Ostrikov, "Self-organized quantum dot arrays: Kinetic mapping of adatom capture," Appl. Phys. Lett. 95, 243102 (2009)

${ }^{157}$ J. P. Franc, "The Rayleigh-Plesset equation: A simple and powerful tool to understand various aspects of cavitation," in Fluid Dynamics of Cavitation and Cavitating Turbopumps, CISM International Centre for Mechanical Sciences, Vol. 496, edited by L. d'Agostino and M. V. Salvetti (Springer, Vienna, 2007).

${ }^{158}$ S. K. Bhangu and M. Ashokkumar, "Theory of sonochemistry," Top. Curr. Chem. 374, 56 (2016).

${ }^{159}$ T. Verreycken, P. Bruggeman, and C. Leys, "Anode pattern formation in atmospheric pressure air glow discharges with water anode," J. Appl. Phys. 105, 083312 (2009).

${ }^{160}$ J. Rooze, M. Andre, G.-J. S. van der Gulik, D. Fernandez-Rivas, J. G. E. Gardeniers, E. V. Rebrov, J. C. Schouten, and J. T. F. Keurentjes, "Hydrodynamic cavitation in micro channels with channel sizes of 100 and 750 micrometers," Microfluid. Nanofluid. 12, 499-508 (2012).

${ }^{161}$ D. Fernandez Rivas and S. Kuhn, "Synergy of microfluidics and ultrasound: Process intensification challenges and opportunities," Top. Curr. Chem. 374, 1-30 (2016)

${ }^{162}$ I. Muller, E. Ammelt, and H.-G. Purwins, "Self-organized quasiparticles: Breathing filaments in a gas discharge system," Phys. Rev. Lett. 82, 3428 (1999).

${ }^{163}$ L. Stollenwerk, "Interaction of current filaments in dielectric barrier discharges with relation to surface charge distributions," New J. Phys. 11, 103034 (2009).

${ }^{164}$ X. Li, P. Zhang, J. Chu, Q. Zhang, X. Lin, P. Jia, and L. Dong, "Surface discharge induced interactions of filaments in argon dielectric barrier discharge at atmospheric pressure," Phys. Plasmas 24, 103520 (2017).

${ }^{165}$ J. P. Trelles, "Pattern formation and self-organization in plasmas interacting with surfaces,” J. Phys. D: Appl. Phys. 49, 393002 (2016).

${ }^{166}$ H.-G. Purwins, H. U. Bödeker, and S. Amiranashvili, "Dissipative solitons," Adv. Phys. 59, 485 (2010).

${ }^{167}$ S. F. Xu and X. X. Zhong, "Non-linear macro evolution of a dc driven micro atmospheric glow discharge," Phys. Plasmas 22, 103519 (2015)

${ }^{168} \mathrm{M}$. Ishaq, K. Bazaka, and K. Ostrikov, "Pro-apoptotic NOXA is implicated in atmospheric-pressure plasma-induced melanoma cell death," J. Phys. D: Appl. Phys. 48, 464002 (2015).

${ }^{169}$ M. Ishaq, K. Bazaka, and K. Ostrikov, "Intracellular effects of atmospheric-pressure plasmas on melanoma cancer cells," Phys. Plasmas 22, 122003 (2015)

${ }^{170}$ M. Ishaq, A. Rowe, K. Bazaka, M. Krockenberger, M. D. Evans, and K. Ostrikov, "Effect of atmospheric-pressure plasmas on drug resistant melanoma: The challenges of translating in vitro outcomes into animal models," Plasma Med. 6, 67-83 (2016).

${ }^{171}$ W. Zhu, S. J. Lee, N. J. Castro, D. Yan, M. Keidar, and L. G. Zhang, "Synergistic effect of cold atmospheric plasma and drug loaded coreshell nanoparticles on inhibiting breast cancer cell growth," Sci. Rep. 6, 21974 (2016).

${ }^{172}$ R. Li, J. Chen, T. C. Cesario, X. Wang, J. S. Yuan, and P. M. Rentzepis, "Synergistic reaction of silver nitrate, silver nanoparticles, and methylene blue against bacteria," Proc. Natl. Acad. Sci. U. S. A. 113, 13612-13617 (2016).

${ }^{173}$ H. Yin, C. Zhang, F. Liu, and Y. Hou, "Hybrid of iron nitride and nitrogen-doped graphene aerogel as synergistic catalyst for oxygen reduction reaction," Adv. Funct. Mater. 24, 2930-2937 (2014).

${ }^{174}$ M. V. Jacob, R. S. Rawat, B. Ouyang, K. Bazaka, D. S. Kumar, D. Taguchi, M. Iwamoto, R. Neupane, and O. K. Varghese, "Catalyst-free plasma enhanced growth of graphene from sustainable sources," Nano Lett. 15, 5702-5708 (2015).

${ }^{175}$ Q. Chen, T. Kitamura, K. Saito, K. Haruta, Y. Yamano, T. Ishikawa, and H. Shirai, "Microplasma discharge in ethanol solution: Characterization and its application to the synthesis of carbon microstructures," Thin Solid Films 516, 4435 (2008).
${ }^{176}$ S. Ghosh, R. Hawtof, P. Rumbach, D. B. Go, R. Akolkar, and R. M. Sankaran, "Quantitative study of electrochemical reduction of $\mathrm{Ag}^{+}$to $\mathrm{Ag}$ nanoparticles in aqueous solutions by a plasma cathode," J. Electrochem. Soc. 164, D818-D824 (2017).

${ }^{177}$ L. O. Snizhko, A. L. Yerokhin, A. Pilkington, N. L. Gurevina, D. O. Misnyankin, A. Leyland, and A. Matthews, "Anodic processes in plasma electrolytic oxidation of aluminium in alkaline solutions," Electrochim. Acta 49, 2085 (2004).

${ }^{178}$ F. Mécuson, T. Czerwiec, G. Henrion, T. Belmonte, L. Dujardin, A. Viola, and J. Beauvir, "Tailored aluminium oxide layers by bipolar current adjustment in the plasma electrolytic oxidation (PEO) process," Surf. Coat. Technol. 201, 8677 (2007).

${ }^{179}$ K. Ariga, Y. Yamauchi, G. Rydzek, Q. Ji, Y. Yonamine, K. C.-W. Wu, and J. P. Hill, "Layer-by-layer nanoarchitectonics: Invention, innovation, and evolution," Chem. Lett. 43, 36-68 (2014).

${ }^{180}$ H. Wang, S. Ishihara, K. Ariga, and Y. Yamauchi, “All-metal layer-bylayer films: Bimetallic alternate layers with accessible mesopores for enhanced electrocatalysis," J. Am. Chem. Soc. 134, 10819 (2012).

${ }^{181}$ H. Ataee-Esfahani, L. Wang, Y. Nemoto, and Y. Yamauchi, "Synthesis of bimetallic Au@Pt nanoparticles with Au core and nanostructured Pt shell toward highly active electrocatalysts," Chem. Mater. 22, 6310 (2010).

${ }^{182}$ C. Richmonds and R. M. Sankaran, "Plasma-liquid electro-chemistry: Rapid synthesis of colloidal metal nanoparticles by microplasma reduction of aqueous cations," Appl. Phys. Lett. 93, 131501 (2008).

${ }^{183}$ W.-H. Chiang, C. Richmonds, and R. M. Sankaran, "Continuous-flow, atmospheric-pressure microplasmas: A versatile source for metal nanoparticle synthesis in the gas or liquid phase," Plasma Sources Sci. Technol. 19, 034011 (2010).

${ }^{184}$ J. Shim, K. Y. Joung, J. H. Ahn, and W. M. Lee, "Carbon-supported platinum nanoparticles synthesized by plasma-chemical reduction method for fuel cell applications," J. Electrochem. Soc. 154, B165 (2007).

${ }^{185}$ R. Zhou, R. Zhou, X. Zhang, J. Zhuang, S. Yang, K. Bazaka et al., "Effects of atmospheric-pressure N 2, He, Air, and $\mathrm{O}_{2}$ microplasmas on Mung bean seed germination and seedling growth," Sci. Rep. 6, 32603 (2016).

${ }^{186}$ K. D. Weltmann, E. Kindel, R. Brandenburg, C. Meyer, R. Bussiahn, C. Wilke, and T. von Woedtke, "Atmospheric pressure plasma jet for medical therapy: Plasma parameters and risk estimation," Contrib. Plasma Phys. 49, 631 (2009)

${ }^{187}$ J. Ahmad, K. Bazaka, J. D. Whittle, A. Michelmore, and M. V. Jacob, "Structural characterization of $\gamma$-terpinene thin films using mass spectroscopy and X-ray photoelectron spectroscopy," Plasma Proc. Polym. 12, 1085-1094 (2015).

${ }^{188}$ J. Ahmad, K. Bazaka, and M. V. Jacob, “Optical and surface characterization of radio frequency plasma polymerized 1-isopropyl-4-methyl-1,4cyclohexadiene thin films," Electronics 3, 266-281 (2014).

${ }^{189}$ J. Ahmad, K. Bazaka, M. Oelgemöller, and M. V. Jacob, "Wetting, solubility and chemical characteristics of plasma-polymerized 1-Isopropyl-4Methyl-1,4-cyclohexadiene thin films," Coatings 4, 527-552 (2014).

${ }^{190}$ C. Turek and F. C. Stintzing, "Stability of essential oils: A review," Compr. Rev. Food Sci. Food Saf. 12, 40 (2013).

${ }^{191}$ U. Neuenschwander, F. Guignard, and I. Hermans, "Mechanism of the aerobic oxidation of alpha-pinene," ChemSusChem 3, 75 (2010).

${ }^{192}$ E. Choe and D. B. Min, Compr. Rev. Food Sci. Food Saf. 5, 169 (2006).

${ }^{193}$ S. Ibrahimkutty, P. Wagener, T. dos Santos Rolo, D. Karpov, A. Menzel, T. Baumbach, S. Barcikowski, and A. Plech, "A hierarchical view on material formation during pulsed-laser synthesis of nanoparticles in liquid," Sci. Rep. 5, 16313 (2015).

${ }^{194}$ H. Kabbara, C. Noël, J. Ghanbaja, K. Hussein, D. Mariotti, V. Švrček, and T. Belmonte, "Synthesis of nanocrystals by discharges in liquid nitrogen from Si-Sn sintered electrode," Sci. Rep. 5, 17477 (2015).

${ }^{195}$ H. Kabbara, J. Ghanbaja, C. Noël, and T. Belmonte, "Synthesis of copper and zinc nanostructures by discharges in liquid nitrogen," Mater. Chem. Phys. 207, 350-358 (2018).

${ }^{196}$ A. Hamdan, C. Noel, F. Kosior, G. Henrion, and T. Belmonte, "Impacts created on various materials by micro-discharges in heptane: Influence of the dissipated charge," J. Appl. Phys. 113, 043301 (2013).

${ }^{197}$ A. Hamdan, J.-N. Audinot, C. Noel, F. Kosior, G. Henrion, and T. Belmonte, "Interaction of micro-discharges in heptane with metallic multi-layers," Appl. Surf. Sci. 274, 378-391 (2013).

${ }^{198}$ P. Wagener, S. Ibrahimkutty, A. Menzel, A. Plech, and S. Barcikowski, "Dynamics of silver nanoparticle formation and agglomeration inside the 
cavitation bubble after pulsed laser ablation in liquid," Phys. Chem. Chem. Phys. 15, 3068-3074 (2013).

${ }^{199}$ J. Fang, I. Aharonovich, I. Levchenko, K. Ostrikov, P. G. Spizzirri, S. Rubanov, and S. Prawer, "Plasma-enabled growth of single-crystalline $\mathrm{SiC} / \mathrm{AlSiC}$ core-shell nanowires on porous alumina templates," Cryst. Growth Des. 12, 2917--2922 (2012).

${ }^{200}$ I. Levchenko, K. Ostrikov, and S. Xu, "Thermodynamical and plasmadriven kinetic growth of high-aspect-ratio nanostructures: Effect of hydrogen termination,” J. Phys. D: Appl. Phys. 42, 125207 (2009).
${ }^{201}$ C. M. Walters, C. Pao, B. P. Gagnon, C. R. Zamecnik, and G. C. Walker, "Bright surface-enhanced Raman scattering with fluorescence quenching from silica encapsulated J-aggregate coated gold nanoparticles," Adv. Mater. 30, 1705381 (2018).

${ }^{202}$ H. R. Griem, "Validity of local thermal equilibrium in plasma spectroscopy," Phys. Rev. 131, 1170 (1963).

${ }^{203}$ H. T. Fujimoto and R. W. P. McWhirter, "Validity criteria for local thermodynamic equilibrium in plasma spectroscopy," Phys. Rev. A 42, 6588 (1990). 